\title{
Two-loop splitting amplitudes and the single-real contribution to inclusive Higgs production at $\mathrm{N}^{3} \mathrm{LO}$
}

\author{
Claude Duhr, ${ }^{a}$ Thomas Gehrmann $^{b}$ and Matthieu Jaquier ${ }^{b}$ \\ ${ }^{a}$ Center for Cosmology, Particle Physics and Phenomenology (CP3), \\ Université Catholique de Louvain, \\ Chemin du Cyclotron 2, B-1348 Louvain-La-Neuve, Belgium \\ ${ }^{b}$ Physik-Institut, Universität Zürich, \\ Wintherturerstrasse 190, CH-8057 Zürich, Switzerland \\ E-mail: claude.duhr@cern.ch, thomas.gehrmann@uzh.ch, \\ matthieu@physik.uzh.ch
}

ABSTRACT: The factorisation of QCD matrix elements in the limit of two external partons
becoming collinear is described by process-independent splitting amplitudes, which can be
expanded systematically in perturbation theory. Working in conventional dimensional reg-
ularisation, we compute the two-loop splitting amplitudes for all simple collinear splitting
processes, including subleading terms in the regularisation parameter. Our results are then
applied to derive an analytical expression for the two-loop single-real contribution to inclu-
sive Higgs boson production in gluon fusion to fourth order $\left(\mathrm{N}^{3} \mathrm{LO}\right)$ in perturbative QCD.

KeYwords: Higgs Physics, QCD

ArXiv EPRINT: 1411.3587 


\section{Contents}

1 Introduction $\quad 1$

2 Collinear limits of multi-loop QCD amplitudes 3

3 Computation of the splitting amplitudes 5

4 Two-loop single-real emission contributions to Higgs production at $\mathrm{N}^{3} \mathrm{LO} \quad 8$

$\begin{array}{lll}4.1 & \text { The } q \bar{q} \text { initial state } & 9\end{array}$

$\begin{array}{lr}4.2 \text { The } q g \text { initial state } & 10\end{array}$

$\begin{array}{lll}4.3 & \text { The } g g \text { initial state } & 11\end{array}$

5 Conclusion $\quad 12$

$\begin{array}{ll}\text { A Splitting amplitudes at two loops } & 13\end{array}$

B The CDR matrix elements for $H$ to three partons $\quad 31$

B.1 The matrix element for $H \rightarrow g g g \quad 31$

B.2 The matrix element for $H \rightarrow q \bar{q} g$

C Two-loop single-real contributions to Higgs production at $\mathrm{N}^{3} \mathrm{LO} \quad \mathbf{3 4}$

C.1 The $g g$ initial state 34

$\begin{array}{ll}\text { C.2 The } g q \text { initial state } & 42\end{array}$

$\begin{array}{ll}\text { C.3 The } q \bar{q} \text { initial state } & 57\end{array}$

\section{Introduction}

Scattering amplitudes in massless QCD diverge if one or more of the external momenta become soft or if two or more external momenta become collinear. In these infrared limits, one observes process-independent factorisation properties: the divergent behaviour of the amplitude is described by universal unresolved factors, multiplying a reduced amplitude with lower partonic multiplicity. These unresolved factors are expanded in a two-fold manner in perturbative QCD: in the number of unresolved partons and in the number of virtual loops correcting the factors at fixed multiplicity. The eikonal factors describing the simple soft behaviour and the collinear splitting amplitudes were established already long ago [1-3]. They are sufficient to understand the infrared singular structure of real radiation contributions at next-to-leading order (NLO) in QCD, and have been instrumental in devising systematic subtraction schemes for NLO calculations [4-6]. They also form the starting point for a systematic expansion around unresolved limits to all orders in perturbation theory [7-11]. 
At next-to-next-to-leading order (NNLO), tree-level contributions with up to two unresolved particles contribute, again displaying universal factorization properties in collinear and soft limits $[12,13]$. At the same order, one-loop corrections to simple collinear [38] and simple soft [14] emissions need to be accounted for. The behaviour in all these limits is understood in detail, and served as a construction principle for subtraction methods at NNLO [15-17]. In an actual calculation of higher order corrections to a collider observable, the universal soft and collinear factors need to be integrated over their appropriate phase spaces, including a regulator for the infrared divergences. Consequently, subleading terms in the regulator will yield finite contributions to the final result: simple soft configurations demand two subleading orders, and simple collinear configurations one subleading order.

With advances in multi-loop calculations, calculations of next-to-next-to-next-toleading order $\left(\mathrm{N}^{3} \mathrm{LO}\right)$ corrections to benchmark observables are now becoming feasible. Multiple unresolved limits at zero and one loop order were understood already some time ago [18-20]. Two-loop corrections to simple soft and simple collinear limits were derived only to finite order in the regulator $[21,22]$ by taking the appropriate limits of the two-loop three-parton decay matrix elements $[23,24]$. With the two-loop soft gluon current recently derived to all orders in the regulator $[25,26], \mathrm{N}^{3} \mathrm{LO}$ calculations at the two-loop single real level are now missing only the two-loop corrections to simple collinear limits, described by the two-loop splitting amplitudes. It is the aim of this paper to derive these, based on a new derivation of the two-loop matrix elements for three-parton decays, expanded to higher orders in the regulator.

The most important application of our newly derived results are the $\mathrm{N}^{3} \mathrm{LO}$ corrections to inclusive Higgs production in gluon fusion. Following the derivation of the three-loop virtual corrections [27-31], of the required renormalization and factorization counterterm contributions [32, 33], and of expansions of single and multiple real radiation contributions around the soft limit $[25,26,34-36]$, the threshold approximation of this coefficient function was computed earlier this year [37]. We use our results for the two-loop splitting amplitudes to derive a closed analytic expression for the two-loop single-real contribution to the inclusive $\mathrm{N}^{3} \mathrm{LO}$ coefficient function for Higgs production. Depending on the availability of results for the other channels (one-loop double-real and triple-real), our expression can be used to derive further terms in the threshold expansion, or to obtain an exact expression for the full coefficient function.

Our paper is structured as follows. In section 2, we establish the notation and discuss the behaviour of multi-loop amplitudes in single collinear limits. Section 3 describes the extraction of the two-loop splitting amplitudes from the calculation of two-loop matrix elements in three-particle decay kinematics. The analytical expressions for the splitting amplitudes are quite lengthy (especially in the term subleading in the dimensional regulator), and are collected in appendix A. These results are then applied in section 4 to compute the two-loop single-real contribution to Higgs production at $\mathrm{N}^{3} \mathrm{LO}$. The two-loop matrix elements relevant to this process were previously known only for external four-dimensional helicity, and are re-derived in conventional dimensional regularization in appendix B, their contribution to the inclusive coefficient function is documented in appendix C. We conclude with an outlook in section 5 . 


\section{Collinear limits of multi-loop QCD amplitudes}

We consider an $\ell$-loop QCD amplitude with a certain number of coloured partons in the final state. Our example will be the decay of a heavy colourless state $X$ into $n$ partons with momenta $p_{i}, i=1, \ldots, n$, and all other cases can be obtained by crossing symmetry. In the limit where a pair of partons become collinear, say $i$ and $j$, then the corresponding Mandelstam invariant vanishes, $s_{i j}=2 p_{i} \cdot p_{j} \rightarrow 0$. The amplitude diverges in the limit, and the divergence is characterised by a simple pole at $s_{i j}=0$. The residue at the pole can be described by the factorisation formula

$$
\begin{aligned}
& \left\langle\mathcal{M}^{(0)}\left(\ldots, p_{i}, p_{j}, \ldots\right) \mid \mathcal{M}^{(\ell)}\left(\ldots, p_{i}, p_{j}, \ldots\right)\right\rangle \\
& \simeq \frac{8 \pi \alpha_{0}}{s_{i j}} \sum_{k=0}^{\ell}\left(\frac{\alpha_{0} S_{\epsilon}}{2 \pi}\right)^{k}\left(\frac{s_{i j}}{\mu^{2}}\right)^{-k \epsilon} e^{k i \pi \epsilon} P_{i j}^{(k)}(z)\left\langle\mathcal{M}^{(0)}(\ldots, P, \ldots) \mid \mathcal{M}^{(\ell-k)}(\ldots, P, \ldots)\right\rangle
\end{aligned}
$$

where $\left|\mathcal{M}^{(\ell)}\left(\ldots, p_{i}, p_{j}, \ldots\right)\right\rangle$ is the $\ell$-loop amplitude and $\alpha_{0}$ the bare strong coupling constant and $P=p_{i}+p_{j}$ the four-momentum of the parent parton. We work in dimensional regularisation in $D=4-2 \epsilon$ dimensions, and

$$
c_{\Gamma}=e^{\gamma_{E} \epsilon} \frac{\Gamma(1-\epsilon)^{2} \Gamma(1+\epsilon)}{\Gamma(1-2 \epsilon)} \text { and } S_{\epsilon}=(4 \pi)^{\epsilon} e^{-\gamma_{E} \epsilon},
$$

with $\gamma_{E}=-\Gamma^{\prime}(1)$ the Euler-Mascheroni constant. The ' $\simeq$ ' sign denotes 'equality up to terms that are power-suppressed in the limit'. The function $P_{i j}^{(\ell)}(z)$ is the $\ell$-loop splitting amplitude, and depends on the colour and spin of the particles involved in the splitting, as well as on the momentum fraction $z$ carried by particle $i, p_{i}=z P$. Note that, in contrast to the well-known Altarelli-Parisi splitting functions, the splitting amplitudes considered in this paper only describe the collinear behaviour of a system of two real partons with momenta $p_{i}$ and $p_{j}$, i.e., they do not include purely virtual 1PI corrections and multiple real radiation corrections. The (spin-averaged) tree-level splitting amplitudes are given by $[1,13]$

$$
\begin{aligned}
& P_{g g}^{(0)}(z)=2 C_{A} \frac{\left(1-z+z^{2}\right)^{2}}{z(1-z)}, \\
& P_{g q}^{(0)}(z)=C_{F}\left[\frac{1+(1-z)^{2}}{z}-\epsilon z\right], \\
& P_{q \bar{q}}^{(0)}(z)=\frac{1}{2}\left[1-2 \frac{z(1-z)}{1-\epsilon}\right],
\end{aligned}
$$

and $P_{q g}^{(0)}(z)=P_{g q}^{(0)}(1-z)$. Here $C_{A}=N$ and $C_{F}=V /(2 N)$ where $N$ is the number of $\mathrm{SU}(N)$ colours and $V=N^{2}-1$ is the number of adjoint colours.

In the remainder of this section we summarise the main properties of splitting amplitudes. It is obvious that Bose symmetry implies

$$
P_{g g}^{(\ell)}(1-z)=P_{g g}^{(\ell)}(z) \text { and } P_{q \bar{q}}^{(\ell)}(1-z)=P_{q \bar{q}}^{(\ell)}(z) .
$$

The limits where $z \rightarrow 0$ or $z \rightarrow 1$ correspond to the limits where one of the particles involved in the splitting becomes soft. The splitting amplitude for $g \rightarrow q \bar{q}$ does obviously 
not have any soft singularity, and so the residue of $P_{q \bar{q}}^{(\ell)}(z)$ at $z=0$ and $z=1$ vanishes. The splitting amplitude $P_{g q}^{(\ell)}(z)$ only develops a soft singularity if the gluon becomes soft, i.e., $z \rightarrow 0$, and the residue at the pole is given by the QCD soft current, known up to two loops in perturbative QCD [2, 3, 14, 22, 25, 26],

$$
P_{g q}^{(\ell)}(z)=\frac{C_{F} c_{\Gamma}^{\ell}}{2^{\ell-1}} z^{-1-\ell \epsilon} r_{S}^{(\ell)}+\mathcal{O}\left(z^{0}\right), \text { as } z \rightarrow 0,
$$

where $r_{S}^{(\ell)}$ denotes the $\ell$-loop QCD soft current (in the normalisation of ref. [25]). Similarly, the gluon splitting amplitude becomes singular if any of the two final-state gluons becomes soft,

$$
\begin{aligned}
P_{g g}^{(\ell)}(z) & =\frac{C_{A} c_{\Gamma}^{\ell}}{2^{\ell-1}} z^{-1-\ell \epsilon} r_{S}^{(\ell)}+\mathcal{O}\left(z^{0}\right), \text { as } z \rightarrow 0, \\
& =\frac{C_{A} c_{\Gamma}^{\ell}}{2^{\ell-1}}(1-z)^{-1-\ell \epsilon} r_{S}^{(\ell)}+\mathcal{O}\left((1-z)^{0}\right), \text { as } z \rightarrow 1
\end{aligned}
$$

The pole structure of $P_{i j}^{(\ell)}(z)$ in dimensional regularisation is completely fixed up to two loops. At one-loop order, we have

$$
P_{i j}^{(1)}(z)=\mathbf{I}_{C, i j}^{(1)}(z, \epsilon) P_{i j}^{(0)}(z)+\mathcal{O}\left(\epsilon^{0}\right)
$$

with

$$
\begin{aligned}
& \mathbf{I}_{C, g g}^{(1)}(z, \epsilon)=-\frac{e^{-\gamma_{E} \epsilon} C_{A}}{2 \epsilon^{2} \Gamma(1-\epsilon)}\left[z^{-\epsilon}+(1-z)^{-\epsilon}-1\right], \\
& \mathbf{I}_{C, g \bar{q}}^{(1)}(z, \epsilon)=-\frac{e^{-\gamma_{E} \epsilon}}{2 \epsilon^{2} \Gamma(1-\epsilon)}\left[N z^{-\epsilon}-\frac{1}{N}\left((1-z)^{-\epsilon}-1\right)\right], \\
& \mathbf{I}_{C, q \bar{q}}^{(1)}(z, \epsilon)=-\frac{e^{-\gamma_{E} \epsilon}}{2 \Gamma(1-\epsilon)}\left[\frac{N}{\epsilon^{2}}\left(z^{-\epsilon}+(1-z)^{-\epsilon}-2\right)-\frac{1}{N \epsilon^{2}}+\frac{3 C_{F}-2 \beta_{0}}{\epsilon}\right],
\end{aligned}
$$

where $\beta_{l}$ is the $l$-loop QCD $\beta$ function. At two-loop order we have [8-11, 22]

$$
P_{i j}^{(2)}(z)=\mathbf{I}_{C, i j}^{(2)}(z, \epsilon) P_{i j}^{(0)}(z)+\mathbf{I}_{C, i j}^{(1)}(z, \epsilon) P_{i j}^{(1)}(z)+\mathcal{O}\left(\epsilon^{0}\right),
$$

with

$$
\begin{aligned}
\mathbf{I}_{C, i j}^{(2)}(z, \epsilon)= & -\frac{1}{2}\left[\mathbf{I}_{C, i j}^{(1)}(z, \epsilon)\right]^{2}-\frac{\beta_{0}}{\epsilon} \mathbf{I}_{C, i j}^{(1)}(z, \epsilon)+\frac{e^{-\gamma_{E} \epsilon} \Gamma(1-2 \epsilon)}{\Gamma(1-\epsilon)}\left(\frac{\beta_{0}}{\epsilon}+K\right) \mathbf{I}_{C, i j}^{(1)}(z, 2 \epsilon) \\
& +\frac{e^{\gamma_{E} \epsilon}}{4 \epsilon \Gamma(1-\epsilon)}\left(H_{i}^{(2)}+H_{j}^{(2)}-H_{i+j}^{(2)}+\beta_{1}-\beta_{0} K\right),
\end{aligned}
$$

where the $H_{l}^{(2)}$ depend on the flavour of the parton,

$$
\begin{aligned}
H_{q}^{(2)}= & \frac{N^{2}-1}{N} N_{f}\left(\frac{1}{8} \zeta_{2}-\frac{25}{216}\right)-\frac{1}{16} \zeta_{2}-\frac{1}{4} \zeta_{3}+N^{2}\left(-\frac{11}{16} \zeta_{2}+\frac{7}{4} \zeta_{3}+\frac{409}{864}\right) \\
& +\frac{1}{N^{2}}\left(\frac{3}{4} \zeta_{2}-\frac{3}{2} \zeta_{3}-\frac{3}{32}\right)-\frac{41}{108} \\
H_{g}^{(2)}= & N N_{f}\left(-\frac{1}{12} \zeta_{2}-\frac{89}{108}\right)+\frac{5}{27} N_{f}^{2}-\frac{N_{f}}{4 N}+N^{2}\left(\frac{11}{24} \zeta_{2}+\frac{1}{2} \zeta_{3}+\frac{5}{12}\right),
\end{aligned}
$$


and

$$
\begin{aligned}
\beta_{0} & =\frac{11 C_{A}-2 N_{f}}{6} \\
\beta_{1} & =\frac{17 C_{A}^{2}-5 C_{A} N_{f}-3 C_{F} N_{f}}{6} \\
K & =\left(\frac{67}{18}-\zeta_{2}\right) C_{A}-\frac{5}{9} N_{f},
\end{aligned}
$$

with $N_{f}$ the number of flavours. We recall that the above expressions refer to the splitting amplitudes describing the collinear behaviour of the unrenormalized one-loop and two-loop matrix elements, as in [22]. Formulae for the factorization of the pole structure can also be derived after renormalization [8-11].

Splitting amplitudes have been computed in the literature up to two-loops. The treelevel and one-loop splitting amplitudes are known to all orders in the dimensional regulator $\epsilon$ (in a variety of schemes) $[1,38]$. The two-loop splitting amplitudes have so far only been computed up to finite terms [21,22]. Our goal is to compute the two-loop splitting amplitudes to $\mathcal{O}(\epsilon)$ in conventional dimensional regularisation (CDR). This result is needed in order to construct the collinear counterterms for the double-virtual-real contributions to the inclusive $\mathrm{N}^{3} \mathrm{LO}$ cross section for the production of a heavy colourless state.

\section{Computation of the splitting amplitudes}

In this section we describe the computation of the two-loop splitting amplitudes to $\mathcal{O}(\epsilon)$ in CDR. The splitting amplitudes can be extracted most conveniently from the two-loop amplitude for a heavy colourless state decaying into three massless partons. In particular, we have

$$
\begin{aligned}
\left\langle\mathcal{M}_{H \rightarrow g g g}^{(0)} \mid \mathcal{M}_{H \rightarrow g g g}^{(\ell)}\right\rangle & \simeq \frac{8 \pi \alpha_{0}}{s_{13}} \sum_{k=0}^{\ell}\left(\frac{\alpha_{0} S_{\epsilon}}{2 \pi}\right)^{k}\left(\frac{s_{13}}{\mu^{2}}\right)^{-k \epsilon} e^{k i \pi \epsilon} P_{g g}^{(k)}(z)\left\langle\mathcal{M}_{H \rightarrow g g}^{(0)} \mid \mathcal{M}_{H \rightarrow g g}^{(\ell-k)}\right\rangle, \\
\left\langle\mathcal{M}_{H \rightarrow q \bar{q} g}^{(0)} \mid \mathcal{M}_{H \rightarrow q \bar{q} g}^{(\ell)}\right\rangle & \simeq \frac{8 \pi \alpha_{0}}{s_{12}} \sum_{k=0}^{\ell}\left(\frac{\alpha_{0} S_{\epsilon}}{2 \pi}\right)^{k}\left(\frac{s_{12}}{\mu^{2}}\right)^{-k \epsilon} e^{k i \pi \epsilon} P_{q \bar{q}}^{(k)}(z)\left\langle\mathcal{M}_{H \rightarrow g g}^{(0)} \mid \mathcal{M}_{H \rightarrow g g}^{(\ell-k)}\right\rangle, \\
\left\langle\mathcal{M}_{\gamma^{*} \rightarrow q \bar{q} g}^{(0)} \mid \mathcal{M}_{\gamma^{*} \rightarrow q \bar{q} g}^{(\ell)}\right\rangle & \simeq \frac{8 \pi \alpha_{0}}{s_{13}} \sum_{k=0}^{\ell}\left(\frac{\alpha_{0} S_{\epsilon}}{2 \pi}\right)^{k}\left(\frac{s_{13}}{\mu^{2}}\right)^{-k \epsilon} e^{k i \pi \epsilon} P_{g q}^{(k)}(z)\left\langle\mathcal{M}_{\gamma^{*} \rightarrow q \bar{q}}^{(0)} \mid \mathcal{M}_{\gamma^{*} \rightarrow q \bar{q}}^{(\ell-k)}\right\rangle,
\end{aligned}
$$

with $z=s_{23} / Q^{2}$, where $Q^{2}$ is the virtuality off the initial state. The hard matrix elements are given by

$$
\begin{aligned}
\left\langle\mathcal{M}_{H \rightarrow g g}^{(0)} \mid \mathcal{M}_{H \rightarrow g g}^{(\ell)}\right\rangle & =\left(\frac{S_{\epsilon} \alpha_{0}}{2 \pi}\right)^{\ell} \frac{\lambda_{0}^{2}\left(N^{2}-1\right)}{2^{\ell+1}}(1-\epsilon) e^{i \pi \ell \epsilon}\left(Q^{2}\right)^{-1-\ell \epsilon} \mathcal{F}_{g}^{(\ell)}, \\
\left\langle\mathcal{M}_{\gamma^{*} \rightarrow q q}^{(0)} \mid \mathcal{M}_{\gamma^{*} \rightarrow q \bar{q}}^{(\ell)}\right\rangle & =\left(\frac{S_{\epsilon} \alpha_{0}}{2 \pi}\right)^{\ell} \frac{e_{q}^{2} N}{2^{\ell-2}}(1-\epsilon) e^{i \pi \ell \epsilon}\left(Q^{2}\right)^{-1-\ell \epsilon} \mathcal{F}_{q}^{(\ell)},
\end{aligned}
$$

where $\lambda_{0}$ and $e_{q}$ denote the bare coupling of the Higgs boson to the gluons (in the large top mass limit) and the bare electromagnetic coupling of the quarks, and $\mathcal{F}_{g}^{(\ell)}$ and $\mathcal{F}_{q}^{(\ell)}$ denote 
the $\ell$-loop gluon and quark form factors, known up to three loops in QCD [27-31]. As a consequence, if we know the the matrix elements in the left-hand side in CDR through $\mathcal{O}(\epsilon)$ up to two loops, we can immediately extract the corresponding splitting amplitudes through $\mathcal{O}(\epsilon)$. The two-loop matrix element for $\gamma^{*} \rightarrow q \bar{q} g$ in CDR was presented to all orders in $\epsilon$ in terms of the master integrals in ref. [23]. In ref. [24] the two-loop $D$ dimensional tensor coefficients as well as the two-loop helicity coefficients (in the 't Hooft Veltman) scheme for $H$ to three partons were presented. In appendix B we show how to construct the matrix elements in CDR from the tensor coefficients of ref. [24].

The two-loop matrix elements are given, to all orders in the dimensional regulator, in terms of the master integrals of refs. [39, 40], where the complete set of master integrals was evaluated in terms of harmonic polylogarithms (HPLs) and two-dimensional harmonic polylogarithms (2dHPLs) [39, 41] up to transcendental weight four. This is sufficient to compute the corresponding matrix elements up to finite terms (some of the master integrals are known to all orders in $\epsilon$, see, e.g., ref. [42]). Since our goal is to compute the splitting amplitudes to $\mathcal{O}(\epsilon)$, we need to compute all the masters integrals to one order higher in the $\epsilon$ expansion, i.e., up to terms of weight five. Moreover, it turns out to be convenient to have the master integrals in a form where all the divergent logarithms in the collinear limit are resummed in $\epsilon$, because the $\ell$-loop splitting amplitude can then immediately be read off from the coefficient of $s_{i j}^{-\ell \epsilon}$ in the residue of the matrix element at $s_{i j}=0$ (and dividing out the hard matrix element, cf. eq. (3.1)). We have therefore explicitly computed all the master integrals of refs. $[39,40]$ up to transcendental weight five using the method of differential equations [42-44]. We choose a canonical basis of master integrals where all the master integrals are of uniform transcendental weight [45]. In this basis, every master integral is a function of three variables $x_{i j}$ (after dividing out an overall scale), defined by

$$
x_{i j}=\frac{s_{i j}}{Q^{2}},
$$

and constrained by $x_{12}+x_{13}+x_{23}=1$. Every uniformly transcendental master integral then admits a representation of the form ${ }^{1}$

$$
U_{i}\left(x_{12}, x_{13}, x_{23} ; \epsilon\right)=\sum_{m, n=0}^{2} x_{13}^{-m \epsilon} x_{23}^{-n \epsilon} \sum_{k=0}^{\infty} \epsilon^{k} u_{i, m, n, k}\left(x_{13}, x_{23}\right),
$$

with $u_{i, m, n, k}\left(x_{13}, x_{23}\right)$ a linear combination of 2 dHPLs with rational coefficients that are analytic in a neighbourhood of $\left(x_{13}, x_{23}\right)=(0,0)$. We have explicitly computed the functions $u_{i, m, n, k}\left(x_{13}, x_{23}\right)$ up to weight $k=5$, which is sufficient to compute the two-loop matrix elements up to order $\epsilon$. The initial conditions for the differential equations were obtained from the leading term in the expansion of the master integrals around the soft limit [25]. We checked that our results agree with the first few subleading terms in the soft expansion of the master integrals.

Once the master integrals are known up to terms of transcendental weight five, we can immediately compute the residue at $x_{13}=0$ by expanding in this variable (while keeping

\footnotetext{
${ }^{1}$ Note that a similar representation obviously exists where any other combination of two out of the three variables $x_{i j}$ are singled out.
} 
the logarithmic divergencies resummed in the form $\left.x_{13}^{-n \epsilon}\right)$. The variable $x_{13}$ only enters the matrix elements through rational functions, as well as through $2 \mathrm{dHPLs}$ of the form $G\left(\vec{a} ; x_{13}\right)$, where $\vec{a}$ is independent of $x_{13}$, and the last entry in $\vec{a}$ is non zero. 2dHPLs of this type can easily be expanded into a power series close to $x_{13}=0$ by using the series representation of multiple polylogarithms,

$$
\begin{aligned}
& G\left(\overrightarrow{0}_{m_{1}-1}, a_{1}, \ldots, \overrightarrow{0}_{m_{k}-1}, a_{k} ; x_{13}\right) \\
& \quad=(-1)^{k} \sum_{n=1}^{\infty} \frac{1}{n^{m_{1}}}\left(\frac{x_{13}}{a_{1}}\right)^{n} Z_{m_{2}, \ldots, m_{k}}\left(n-1 ; \frac{a_{1}}{a_{2}}, \ldots, \frac{a_{k-1}}{a_{k}}\right),
\end{aligned}
$$

with $\overrightarrow{0}_{m}=(\underbrace{0, \ldots, 0}_{m \text { times }})$ and the $Z_{m_{2}, \ldots, m_{k}}$ denote $Z$ sums [46]. The splitting amplitudes up to two-loops and up to $\mathcal{O}(\epsilon)$ can then immediately be read off by comparing the expression to eq. (3.1). At one loop we find

$$
\begin{aligned}
P_{i j}^{(1)}(z)= & -C_{A} \frac{\Gamma(1+\epsilon) \Gamma(1-\epsilon)}{\epsilon^{2}} P_{i j}^{(0)}(z) z^{-\epsilon}(1-z)^{\epsilon} \\
& +2 P_{i j}^{(0)}(z)\left[C_{i+j} A^{(1)}(z, \epsilon)+\left(C_{i+j}-C_{A}\right) B^{(1)}(z, \epsilon)\right]+R_{i j}^{(1)}(z, \epsilon),
\end{aligned}
$$

where $C_{i+j}$ is the Casimir of $\mathrm{SU}(N)$ in the representation of the parent parton, and $A^{(1)}(z, \epsilon)$ and $B^{(1)}(z, \epsilon)$ are functions that are analytic close to $z=0$. They are independent of the identities of the partons involved in the splitting and can be written as a combination of harmonic polylogarithms of uniform weight,

$$
\begin{aligned}
A^{(1)}(z, \epsilon)=- & \frac{1}{\epsilon} H_{1}-\epsilon\left(H_{2,1}+H_{1,2}+H_{1,1,1}+H_{3}\right)-\epsilon^{3}\left(H_{4,1}+H_{3,2}+H_{3,1,1}+H_{2,3}\right. \\
& +H_{2,2,1}+H_{2,1,2}+H_{2,1,1,1}+H_{1,4}+H_{1,3,1}+H_{1,2,2}+H_{1,2,1,1}+H_{1,1,3} \\
& \left.+H_{1,1,2,1}+H_{1,1,1,2}+H_{1,1,1,1,1}+H_{5}\right)+\mathcal{O}\left(\epsilon^{4}\right), \\
B^{(1)}(z, \epsilon)= & -\frac{1}{\epsilon^{2}}-\left(H_{1,1}+H_{2}\right)-\epsilon^{2}\left(H_{3,1}+H_{2,2}+H_{2,1,1}+H_{1,3}+H_{1,2,1}+H_{1,1,2}\right. \\
& \left.+H_{1,1,1,1}+H_{4}\right)+\mathcal{O}\left(\epsilon^{3}\right),
\end{aligned}
$$

where we used a shorthand for harmonic polylogarithms, $H_{i, \ldots, j} \equiv H_{i, \ldots, j}(z)$. The function $R_{i j}^{(1)}(z, \epsilon)$ are rational functions of $z$ order by order in the $\epsilon$ expansion and depend on the identity of the partons involved in the splitting,

$$
\begin{aligned}
R_{g g}^{(1)}(z, \epsilon)= & \frac{N}{3}[1-2 \epsilon z(1-z)]\left[N-N_{f}+\frac{\epsilon}{3}\left(11 N-14 N_{f}\right)+\frac{\epsilon^{2}}{9}\left(85 N-127 N_{f}\right)\right. \\
& \left.+\frac{\epsilon^{3}}{27}\left(575 N-956 N_{f}\right)+\mathcal{O}\left(\epsilon^{4}\right)\right], \\
R_{g q}^{(1)}(z, \epsilon)= & \frac{N^{4}-1}{8 N^{2}}\left(\frac{2-z}{1-2 \epsilon}+z\right)+\mathcal{O}\left(\epsilon^{4}\right), \\
R_{q q}^{(1)}(z, \epsilon)= & P_{q q}^{(0)}(z) \frac{1}{2 \epsilon(1-2 \epsilon)}\left(N \frac{13-8 \epsilon}{3-2 \epsilon}-4 N_{f} \frac{1-\epsilon}{3-2 \epsilon}+\frac{3+2 \epsilon}{N}\right)+\mathcal{O}\left(\epsilon^{4}\right) .
\end{aligned}
$$

We emphasise that the previous expressions are valid for a time-like splitting where the collinear pair is in the final state. In the case of only two hard partons (as is the case for 
the matrix elements considered in this section) the analytic continuation to a space-like splitting can easily be performed by the replacement

$$
z^{-k \epsilon} \rightarrow e^{-i \pi k \epsilon} z^{-k \epsilon}
$$

in eq. (3.6). We note that for space-like splitting involving more than two hard partons, collinear factorisation is expected to be violated [47]. At two loops, we write

$$
P_{i j}^{(2)}(z)=\sum_{k=0}^{2} z^{-k \epsilon} P_{i j}^{(2, k)}(z),
$$

where $P_{i j}^{(2, k)}(z)$ are analytic in a neighbourhood of $z=0$. Their analytical expressions are given in appendix A.

We checked that the tree-level and one-loop are correctly reproduced from our two-loop computation. Moreover, we checked that our results have the correct symmetry properties and soft limits, eq. (2.4)-(2.6), as well as the the correct pole structure in dimensional regularisation, eq. (2.10).

\section{Two-loop single-real emission contributions to Higgs production at $\mathrm{N}^{3} \mathrm{LO}$}

As an application of the splitting amplitudes up to two loops computed in the previous section, we present in this section the integration over phase space of the matrix elements for the scattering processes

$$
g g \rightarrow H g, \quad q \bar{q} \rightarrow H g, \quad q g \rightarrow H q
$$

These processes are important because they contribute to the inclusive Higgs production cross section at $\mathrm{N}^{3} \mathrm{LO}$ in perturbative QCD. Indeed, the final-state parton may become collinear to any of the two incoming partons, and the matrix elements develop singularities which are described by the splitting amplitudes we have just computed. The knowledge of the splitting amplitudes therefore enables us to write down explicit counterterms that we can subtract from the matrix element in order to render the phase-space integrations finite. Moreover, the counterterms will turn out to be trivial to integrate over the unresolved phase space, giving rise to a pole in dimensional regularisation. This pole, in turn, is at the origin of the appearance of the $\mathcal{O}(\epsilon)$ part of the splitting amplitudes computed in the previous section in the finite terms which enter the cross section. We start by giving some generic considerations about the phase-space parametrisation we will use in the following to perform the integration, and we discuss the different channels separately in subsequent subsections.

We denote the momenta of the two incoming partons by $q_{1}$ and $q_{2}$, and the momentum of the final-state parton is denoted by $q_{3}$. Furthermore, we define

$$
s=2 q_{1} \cdot q_{2}, \quad t=2 q_{1} \cdot q_{3}, \quad u=2 q_{2} \cdot q_{3} .
$$

Note that $s, t, u>0$ and $s=m_{H}^{2}+t+u$. The phase space integrals we want to compute are

$$
\frac{\mathcal{N}_{X}}{2 s} \int d \Phi_{2} 2 \operatorname{Re}\left\langle\mathcal{M}_{X}^{(0)} \mid \mathcal{M}_{X}^{(2)}\right\rangle
$$


where $X$ denotes the channel under consideration, and $\mathcal{N}_{X}$ denotes the averaging factor over the initial-state colours and spins,

$$
\mathcal{N}_{g g \rightarrow H g}=\frac{1}{4 V^{2}(1-\epsilon)^{2}}, \quad \mathcal{N}_{q g \rightarrow H q}=\frac{1}{4 N^{2}(1-\epsilon)}, \quad \mathcal{N}_{q \bar{q} \rightarrow H g}=\frac{1}{4 N V} .
$$

The $D$-dimensional phase-space measure is given by

$$
d \Phi_{2}=(2 \pi)^{D} \delta^{(D)}\left(q_{H}+q_{3}-q_{1}-q_{2}\right) \frac{d^{D} q_{H}}{(2 \pi)^{D-1}} \delta_{+}\left(q_{H}^{2}-m_{H}^{2}\right) \frac{d^{D} q_{3}}{(2 \pi)^{D-1}} \delta_{+}\left(q_{3}^{2}\right) .
$$

We parametrise the phase space by

$$
m_{H}^{2}=(1-\bar{z}), \quad t=s \bar{z} \lambda, \quad u=s \bar{z}(1-\lambda) .
$$

In this parametrization the phase-space measure becomes

$$
d \Phi_{2}=\frac{S_{\epsilon} e^{\gamma_{E} \epsilon} s^{-\epsilon} \bar{z}^{1-2 \epsilon}}{8 \pi \Gamma(1-\epsilon)} d \lambda[\lambda(1-\lambda)]^{-\epsilon} \Theta(\lambda) \Theta(1-\lambda),
$$

where $\Theta$ denotes the step function. In the following we discuss the integration over phase space of the three different processes in eq. (4.1), and we separately discuss the processes with different initial states.

\subsection{The $q \bar{q}$ initial state}

The two-loop matrix element for $q \bar{q} \rightarrow H g$ can be written as, with $s=\mu^{2}=1$

$$
\begin{aligned}
\left\langle\mathcal{M}_{q \bar{q} \rightarrow H g}^{(0)} \mid \mathcal{M}_{q \bar{q} \rightarrow H g}^{(2)}\right\rangle= & 8 \pi^{2} \lambda_{0}^{2}\left(\frac{\alpha_{0}}{2 \pi}\right)^{3}\left(\frac{S_{\epsilon} c_{\Gamma}}{2}\right)^{2} C_{F} C_{A} e^{2 i \pi \epsilon}(1-\bar{z})^{1-2 \epsilon} \\
& \times M_{q \bar{q} g}^{(2)}\left(1+x_{t}+x_{u},-x_{t},-x_{u} ; \epsilon\right),
\end{aligned}
$$

with

$$
x_{t}=\frac{t}{m_{H}^{2}}=s \frac{\bar{z}}{1-\bar{z}} \lambda \text { and } x_{u}=\frac{u}{m_{H}^{2}}=s \frac{\bar{z}}{1-\bar{z}}(1-\lambda)
$$

and $M_{q \bar{q} g}^{(2)}$ is a Laurent series in $\epsilon$ whose coefficients are rational functions of the $x_{i j}$ multiplied by 2dHPLs. The expression of $M_{q \bar{q} g}^{(2)}$ in terms of the tensor coefficients of ref. [24] can be found in appendix B. Note that $M_{q \bar{q} g}^{(2)}$ develops an imaginary part, because the master integrals have branch points at $x_{t}=0$ and $x_{u}=0$,

$$
U_{i}\left(1+x_{t}+x_{u},-x_{t},-x_{u} ; \epsilon\right)=\sum_{m, n=0}^{2} e^{-(m+n) i \pi \epsilon} x_{t}^{-m \epsilon} x_{u}^{-n \epsilon} \sum_{k=0}^{\infty} \epsilon^{k} u_{i, m, n, k}\left(-x_{t},-x_{u}\right) .
$$

The functions $u_{i, m, n, k}\left(x_{t}, x_{u}\right)$ are real, because they are analytic in a neighbourhood of $\left(x_{t}, x_{u}\right)=(0,0)$. In the previous section, we argued that the amplitude is only singular if the quark pair becomes collinear. This implies that $\left\langle\mathcal{M}_{q \bar{q} \rightarrow H g}^{(0)} \mid \mathcal{M}_{q \bar{q} \rightarrow H g}^{(2)}\right\rangle$ is finite everywhere inside the phase space, and so we can simply expand in $\epsilon$ and perform the integral over $\lambda$ order by order in $\epsilon$. The integrand contains 2 dHPLs of the form $G\left(\vec{a}\left(x_{u}\right) ;-x_{t}\right)$, where $x_{t}$ and $x_{u}$ 
are rational functions of $\lambda$ and $\bar{z}$ (cf. eq. (4.6)). Interpreting the 2dHPLs as special instances of multiple polylogarithms [48], and using the techniques of refs. [49-56], we can easily convert all the 2dHPLs of the form $G\left(\vec{a}\left(x_{u}\right) ;-x_{t}\right)$ into multiple polylogarithms of the form $G(\vec{b}(\bar{z}) ; \lambda)$ and multiple polylogarithms in $\bar{z}$. As an example, the following relation holds

$$
\begin{aligned}
G\left(\frac{(\lambda-1) \bar{z}}{\bar{z}-1}, 0,1 ; \frac{\lambda \bar{z}}{\bar{z}-1}\right)= & -G\left(1,0, \frac{\bar{z}-1}{\bar{z}} ; \lambda\right)-G\left(1, \frac{2 \bar{z}-1}{\bar{z}}, 1 ; \lambda\right) \\
& -G\left(1, \frac{2 \bar{z}-1}{\bar{z}}, \frac{\bar{z}-1}{\bar{z}} ; \lambda\right) .
\end{aligned}
$$

Similar identities can easily be derived in all other cases, and the phase-space integration over $\lambda$ can easily be performed using the the definition of the multiple polylogarithms,

$$
\int_{0}^{1} \frac{d \lambda}{\lambda-b_{1}(\bar{z})} G(\vec{b}(\bar{z}) ; \lambda)=G\left(b_{1}(\bar{z}), \vec{b}(\bar{z}) ; 1\right)
$$

After a final massaging, we find that all the phase space integrals can be expressed in terms of HPLs in $1-\bar{z}$, in agreement with the expectations.

\subsection{The $q g$ initial state}

The two-loop matrix element for $q g \rightarrow H q$ can be written as, with $s=\mu^{2}=1$,

$$
\begin{aligned}
\left\langle\mathcal{M}_{q g \rightarrow H q}^{(0)} \mid \mathcal{M}_{q g \rightarrow H q}^{(2)}\right\rangle= & -8 \pi^{2} \lambda_{0}^{2}\left(\frac{\alpha_{0}}{2 \pi}\right)^{3}\left(\frac{S_{\epsilon} c_{\Gamma}}{2}\right)^{2} C_{F} C_{A} e^{2 i \pi \epsilon}(1-\bar{z})^{1-2 \epsilon} \\
& \times M_{q \bar{q} g}^{(2)}\left(-x_{t}, 1+x_{t}+x_{u},-x_{u} ; \epsilon\right)
\end{aligned}
$$

The function $M_{q \bar{q} g}^{(2)}\left(-x_{t}, 1+x_{t}+x_{u},-x_{u} ; \epsilon\right)$ is identical to the corresponding quantity in the $q \bar{q} \rightarrow H g$ case, up to analytic continuation. Note that there is an additional minus sign, coming from the crossing of a single fermion to the initial state. The analytic continuation of the function $M_{q \bar{q} g}^{(2)}\left(-x_{t}, 1+x_{t}+x_{u},-x_{u} ; \epsilon\right)$ can be performed in a similar way as in the $q \bar{q} \rightarrow H g$ case.

The integrand becomes singular when the initial and final-state quarks are collinear, and the singularity is controlled by the splitting amplitudes computed in section 3. More precisely, after inserting the parametrisation (4.2), the integrand has a simple pole as $\lambda \rightarrow 0$, and from eq. (3.1), we have

$$
\left\langle\mathcal{M}_{q g \rightarrow H q}^{(0)} \mid \mathcal{M}_{q g \rightarrow H q}^{(2)}\right\rangle=\sum_{k=0}^{2} \lambda^{-1-k \epsilon} \mathbf{C}_{q \bar{q}}^{(2, k)}(\bar{z} ; \epsilon)+\mathcal{O}\left(\lambda^{0}\right)
$$

with

$$
\mathbf{C}_{q \bar{q}}^{(2, k)}(\bar{z} ; \epsilon)=16 \pi^{2}\left(\frac{\alpha_{0}}{2 \pi}\right)^{k+1} S_{\epsilon}^{k} \bar{z}^{-1-k \epsilon} e^{-k i \pi \epsilon} P_{q \bar{q}}^{(k)}\left(\frac{\bar{z}}{\bar{z}-1}\right)\left\langle\mathcal{M}_{g g \rightarrow H}^{(0)} \mid \mathcal{M}_{g g \rightarrow H}^{(2-k)}\right\rangle .
$$


Note that the splitting amplitude is evaluated at $\lambda=0$, so that we only subtract the residue of the matrix element at $\lambda=0$. The phase-space integral then becomes

$$
\begin{aligned}
& \int_{0}^{1} d \lambda[\lambda(1-\lambda)]^{-\epsilon}\left\langle\mathcal{M}_{q g \rightarrow H q}^{(0)} \mid \mathcal{M}_{q g \rightarrow H q}^{(2)}\right\rangle=-\frac{1}{\epsilon} \sum_{k=0}^{2} \frac{\mathbf{C}_{q \bar{q}}^{(2, k)}(\bar{z} ; \epsilon)}{(k+1)} \\
& \quad+\int_{0}^{1} d \lambda\left\{[\lambda(1-\lambda)]^{-\epsilon}\left\langle\mathcal{M}_{q g \rightarrow H q}^{(0)} \mid \mathcal{M}_{q g \rightarrow H q}^{(2)}\right\rangle-\sum_{k=0}^{2} \lambda^{-1-(k+1) \epsilon} \mathbf{C}_{q \bar{q}}^{(2, k)}(\bar{z} ; \epsilon)\right\}
\end{aligned}
$$

The integral in the second line is convergent, and so we can expand in $\epsilon$ under the integration sign and integrate order by order in $\epsilon$. At this point, however, we need to face a technical difficulty: performing the integrations using eq. (4.12) requires the integrations to be performed term by term. In general, these integrals will be divergent, because only the sum in the second line of eq. (4.16) is finite. We therefore introduce a cut-off $\delta$ for the lower integration limit ${ }^{2}$ and perform all the integrations term by term. As a result, all the integrals are convergent, but they explicitly depend on the cut-off. We observe that all divergences cancel when we expand the result around $\delta=0$, in agreement with the expectation that the integral in the second line of eq. (4.16) is convergent.

\subsection{The $g g$ initial state}

The two-loop matrix element for $g g \rightarrow H g$ can be written as

$$
\begin{aligned}
\left\langle\mathcal{M}_{g g \rightarrow H g}^{(0)} \mid \mathcal{M}_{g g \rightarrow H g}^{(2)}\right\rangle= & 8 \pi^{2} s^{1-2 \epsilon}\left|\lambda_{0}\right|^{2}\left(\frac{\alpha_{0}}{2 \pi}\right)^{3}\left(\frac{S_{\epsilon} c_{\Gamma}}{2}\right)^{2} e^{2 i \pi \epsilon}(1-\bar{z})^{1-2 \epsilon} V C_{A} \\
& \times M_{g g g}^{(2)}\left(1+x_{t}+x_{u},-x_{t},-x_{u} ; \epsilon\right) .
\end{aligned}
$$

The expression of $M_{g g g}^{(2)}$ in terms of the tensor coefficients of ref. [24] is given in appendix B. Its analytic continuation can be performed in the same way as in the previous cases. The phase-space integral diverges in the collinear limits where either $t$ or $u$ vanish. Moreover, the gluon amplitude also develops a singularity when the final state gluon becomes soft, corresponding to a simultaneous vanishing of $t$ and $u$. In terms of our phase space parametrisation (4.6) this corresponds to $\bar{z} \rightarrow 0$, and in this limit the matrix element behaves like

$$
\left\langle\mathcal{M}_{g g \rightarrow H g}^{(0)} \mid \mathcal{M}_{g g \rightarrow H g}^{(2)}\right\rangle=\sum_{k=0}^{2} \bar{z}^{-2-2 k \epsilon}[\lambda(1-\lambda)]^{-1-k \epsilon} \mathbf{S}_{g}^{(2, k)}(\epsilon)+\mathcal{O}\left(\bar{z}^{0}\right),
$$

with

$$
\mathbf{S}_{g}^{(2, k)}(\epsilon)=-32 \pi^{2}\left(\frac{\alpha_{0}}{2 \pi}\right)^{k+1}\left(\frac{S_{\epsilon}}{2}\right)^{k} C_{A} e^{-k \pi i \epsilon} r_{S}^{(k)}(\epsilon)\left\langle\mathcal{M}_{g g \rightarrow H}^{(0)} \mid \mathcal{M}_{g g \rightarrow H}^{(2-k)}\right\rangle_{\bar{z}=0} .
$$

We define the soft-regularized amplitude, defined as the amplitude with the soft singularity at $\bar{z}=0$ subtracted,

$$
\left\langle\mathcal{M}_{g g \rightarrow H g}^{(0)} \mid \mathcal{M}_{g g \rightarrow H g}^{(2)}\right\rangle^{\mathrm{reg}} \equiv\left\langle\mathcal{M}_{g g \rightarrow H g}^{(0)} \mid \mathcal{M}_{g g \rightarrow H g}^{(2)}\right\rangle-\sum_{k=0}^{2} \bar{z}^{-2-2 k \epsilon}[\lambda(1-\lambda)]^{-1-k \epsilon} \mathbf{S}_{g}^{(2, k)}(\epsilon) .
$$

\footnotetext{
${ }^{2}$ The integrand does not develop poles for $\lambda \rightarrow 1$, and so no cut-off is required for the upper integration limit.
} 
The soft-regularised amplitude is free of soft singularities, but still contains non-overlapping collinear divergences. The residues at the collinear poles at $\lambda=0$ and $\lambda=1$ can be obtained in a way similar to the $q g \rightarrow H q$ case considered in the previous section. By Bose symmetry, the residues at the two poles are identical. In particular, the residue at $\lambda=0$ is given by

$$
\left\langle\mathcal{M}_{g g \rightarrow H g}^{(0)} \mid \mathcal{M}_{g g \rightarrow H g}^{(2)}\right\rangle^{\mathrm{reg}}=\sum_{k=0}^{2} \lambda^{-1-k \epsilon} \mathbf{C}_{g \bar{g}}^{(2, k), \mathrm{reg}}(\bar{z} ; \epsilon)+\mathcal{O}\left(\lambda^{0}\right),
$$

with

$$
\mathbf{C}_{g g}^{(2, k), \operatorname{reg}}(\bar{z} ; \epsilon)=\mathbf{C}_{g g}^{(2, k)}(\bar{z} ; \epsilon)-\bar{z}^{-1-2 k \epsilon} \mathbf{S}_{g}^{(2, k)}(\epsilon),
$$

and

$$
\mathbf{C}_{g g}^{(2, k)}(\bar{z} ; \epsilon)=-16 \pi^{2}\left(\frac{\alpha_{0}}{2 \pi}\right)^{k+1} S_{\epsilon}^{k} \bar{z}^{-1-k \epsilon} e^{-k i \pi \epsilon} P_{g g}^{(k)}\left(\frac{\bar{z}}{\bar{z}-1}\right)\left\langle\mathcal{M}_{g g \rightarrow H}^{(0)} \mid \mathcal{M}_{g g \rightarrow H}^{(2-k)}\right\rangle
$$

The phase-space integral can now be written as

$$
\begin{aligned}
\bar{z}^{1-2 \epsilon} \int_{0}^{1} d \lambda[\lambda(1-\lambda)]^{-\epsilon}\left\langle\mathcal{M}_{g g \rightarrow H g}^{(0)} \mid \mathcal{M}_{g g \rightarrow H g}^{(2)}\right\rangle \\
=\sum_{k=0}^{2} \bar{z}^{-1-2(k+1) \epsilon} \frac{\Gamma(-(k+1) \epsilon)^{2}}{\Gamma(-2(k+1) \epsilon)} \mathbf{S}_{g}^{(2, k)}(\epsilon) \\
\quad+\bar{z}^{1-2 \epsilon} \int_{0}^{1} d \lambda[\lambda(1-\lambda)]^{-\epsilon}\left\langle\mathcal{M}_{g g \rightarrow H g}^{(0)} \mid \mathcal{M}_{g g \rightarrow H g}^{(2)}\right\rangle^{\mathrm{reg}} \\
=\sum_{k=0}^{2}\left\{\bar{z}^{-1-2(k+1) \epsilon} \frac{\Gamma(-(k+1) \epsilon)^{2}}{\Gamma(-2(k+1) \epsilon)} \mathbf{S}_{g}^{(2, k)}(\epsilon)-\frac{2 \bar{z}^{1-2 \epsilon}}{\epsilon} \frac{\mathbf{C}_{g g}^{(2, k), \mathrm{reg}}(\bar{z} ; \epsilon)}{(k+1)}\right\} \\
\quad+\bar{z}^{1-2 \epsilon} \int_{0}^{1} d \lambda\left\{[\lambda(1-\lambda)]^{-\epsilon}\left\langle\mathcal{M}_{g g \rightarrow H g}^{(0)} \mid \mathcal{M}_{g g \rightarrow H g}^{(2)}\right\rangle^{\mathrm{reg}}\right. \\
\quad-\sum_{k=0}^{2}\left[\lambda^{-1-(k+1) \epsilon}+(1-\lambda)^{-1-(k+1) \epsilon]} \mathbf{C}_{g g}^{(2, k), \mathrm{reg}}(\bar{z} ; \epsilon)\right\},
\end{aligned}
$$

The remaining integral is convergent and can be performed order by order in $\epsilon$ using eq. (4.12). Individual terms in the integrand can develop poles as $\lambda$ approaches either 0 or 1. We therefore introduce a cut-off for each integration limit, and we observe that all the singularities cancel in the sum over all terms when the cut-offs are removed, confirming the expectation that the remaining integral in eq. (4.24) is convergent.

\section{Conclusion}

The single collinear factorization of scattering amplitudes in massless QCD is determined by so-called splitting amplitudes, which can be expanded perturbatively in the number of virtual loops [7]. We derived the two-loop corrections to all splitting amplitudes, finding agreement with previously known results $[21,22]$ up to finite terms in the dimensional regulator, and deriving the subleading terms for the first time. When integrated over the unresolved single collinear phase space in actual $\mathrm{N}^{3} \mathrm{LO}$ calculations, these subleading terms produce 
finite contributions. We used our result to analytically compute the two-loop single-real contribution to the coefficient function of inclusive Higgs boson production in gluon fusion, improving upon the previously available soft approximation $[25,26]$ of this function.

When combined with either expanded or full results for the other channels (one-loop double-real and triple-real), our expression will allow to derive further terms in the threshold expansion [37] or to complete an exact calculation of the full $\mathrm{N}^{3} \mathrm{LO}$ coefficient function for Higgs production in gluon fusion.

\section{Acknowledgments}

We would like to thank Falko Dulat and Bernhard Mistlberger [57] as well as Lance Dixon and Hua-Xing Zhu for useful discussions and comparisons with closely related results prior to publication. This research was supported in part by the Swiss National Science Foundation (SNF) under contract 200020-149517 and by the Research Executive Agency (REA) of the European Union under the Grant Agreements PITN-GA-2010-264564 (LHCPhenoNet), PITN-GA-2012-316704 (HiggsTools), and the ERC Advanced Grant MC@NNLO (340983).

\section{A Splitting amplitudes at two loops}

The two-loop splitting amplitudes can be expanded according to the powers $z^{-k \epsilon}$, with coefficients that are analytic around $z=0$, as defined in (3.10). We write these coefficients as:

$$
\begin{aligned}
P_{i j}^{(2,2)}(z)= & P_{i j}^{(0)}(z) U_{i j}^{(2,2)}(z, \epsilon)+V_{i j}^{(2,2)}(z, \epsilon) \\
& +\left(\frac{N}{4 \epsilon^{3}} \beta_{0}-\frac{N}{2 \epsilon^{2}} K\right) P_{i j}^{(0)}(z)(1-z)^{2 \epsilon}, \\
P_{i j}^{(2,1)}(z)= & P_{i j}^{(0)}(z) U_{i j}^{(2,1)}(z, \epsilon)+V_{i j}^{(2,1)}(z, \epsilon) \\
& -\frac{N}{\epsilon^{2}} \Gamma(1-\epsilon) \Gamma(1+\epsilon) R_{i j}^{(1)}(z, \epsilon)(1-z)^{\epsilon}, \\
P_{i j}^{(2,0)}(z)= & P_{i j}^{(0)}(z) U_{i j}^{(2,0)}(z, \epsilon)+V_{i j}^{(2,0)}(z, \epsilon)-\frac{N}{\epsilon^{2}} \beta_{0} P_{i j}^{(0)}(z) H_{1} .
\end{aligned}
$$

Note that these expressions are again valid for a time-like splitting, and the space-like case can again be obtained by the replacement (3.9). The functions $U_{i j}^{(2, k)}(z)$ are of uniform weight, and are given by

$$
\begin{aligned}
U_{g g}^{(2,2)}(z, \epsilon)= & N^{2}\left[\frac{1}{2 \epsilon^{4}}-\frac{1}{\epsilon^{3}} H_{1}+\frac{1}{\epsilon^{2}}\left(2 H_{1,1}+\zeta_{2}\right)+\frac{1}{\epsilon}\left(-4 H_{1,1,1}+\frac{1}{2} \zeta_{3}-2 \zeta_{2} H_{1}\right)\right. \\
& +3 \zeta_{2} H_{1,1}+2 H_{3,1}+3 H_{2,2}+H_{2,1,1}+2 H_{1,3}+H_{1,2,1}+H_{1,1,2}+\frac{29}{4} \zeta_{4} \\
& +8 H_{1,1,1,1}-\zeta_{2} H_{2}+2 \zeta_{3} H_{1}+4 H_{4}+\epsilon\left(-\zeta_{2} H_{1,2}-5 \zeta_{2} H_{1,1,1}\right. \\
& +10 H_{3,2}-3 H_{3,1,1}+10 H_{2,3}-4 H_{2,2,1}-3 H_{2,1,1,1}+8 H_{1,4}-2 H_{1,3,1} \\
& -H_{1,2,2}-4 H_{1,2,1,1}+2 H_{1,1,3}-2 H_{1,1,2,1}-3 H_{1,1,1,2}-16 H_{1,1,1,1,1} \\
& \left.+4 \zeta_{2} \zeta_{3}-\frac{37}{2} \zeta_{5}-4 \zeta_{2} H_{3}-2 \zeta_{3} H_{2}-\frac{35}{4} \zeta_{4} H_{1}+24 H_{5}-12 \zeta_{3} H_{1,1}\right)
\end{aligned}
$$




$$
\begin{aligned}
& \left.+\mathcal{O}\left(\epsilon^{2}\right)\right] \\
& U_{g g}^{(2,1)}(z, \epsilon)=N^{2}\left[\frac{2}{\epsilon^{3}} H_{1}-\frac{4}{\epsilon^{2}} H_{1,1}+\frac{1}{\epsilon}\left(2 H_{2,1}+2 H_{1,2}+8 H_{1,1,1}+2 \zeta_{2} H_{1}+2 H_{3}\right)\right. \\
& -4 \zeta_{2} H_{1,1}-4 H_{3,1}-2 H_{2,2}-6 H_{2,1,1}-2 H_{1,3}-6 H_{1,2,1}-4 H_{1,1,2} \\
& -16 H_{1,1,1,1}-12 \zeta_{3} H_{1}+\epsilon\left(2 \zeta_{2} H_{2,1}+2 \zeta_{2} H_{1,2}+8 \zeta_{2} H_{1,1,1}+24 \zeta_{3} H_{1,1}\right. \\
& +2 H_{4,1}+2 H_{3,2}+8 H_{3,1,1}+2 H_{2,3}+6 H_{2,2,1}+4 H_{2,1,2}+14 H_{2,1,1,1} \\
& +2 H_{1,4}+6 H_{1,3,1}+4 H_{1,2,2}+14 H_{1,2,1,1}+4 H_{1,1,3}+12 H_{1,1,2,1}+8 H_{1,1,1,2} \\
& \left.\left.+32 H_{1,1,1,1,1}+2 \zeta_{2} H_{3}-\frac{29}{2} \zeta_{4} H_{1}+2 H_{5}\right)+\mathcal{O}\left(\epsilon^{2}\right)\right] \\
& U_{g g}^{(2,0)}(z, \epsilon)=N^{2}\left[\frac{4}{\epsilon^{2}} H_{1,1}+\frac{2}{\epsilon} \zeta_{2} H_{1}+\zeta_{2} H_{1,1}+6 H_{3,1}+H_{2,2}+11 H_{2,1,1}+2 H_{1,3}\right. \\
& +11 H_{1,2,1}+7 H_{1,1,2}+16 H_{1,1,1,1}+\zeta_{2} H_{2}+11 \zeta_{3} H_{1}-4 H_{4} \\
& +\epsilon\left(-3 \zeta_{2} H_{1,2}-3 \zeta_{2} H_{1,1,1}-2 \zeta_{3} H_{1,1}+12 H_{4,1}+8 H_{3,2}+7 H_{3,1,1}+8 H_{2,3}\right. \\
& +8 H_{2,2,1}+8 H_{2,1,2}+3 H_{2,1,1,1}+6 H_{1,4}+6 H_{1,3,1}+11 H_{1,2,2}+4 H_{1,2,1,1} \\
& \left.\left.+6 H_{1,1,3}+2 H_{1,1,2,1}+11 H_{1,1,1,2}-2 \zeta_{3} H_{2}+\frac{11}{4} \zeta_{4} H_{1}+8 H_{5}\right)+\mathcal{O}\left(\epsilon^{2}\right)\right] \text {, } \\
& U_{g q}^{(2,2)}(z, \epsilon)=N^{2}\left[\frac{1}{2 \epsilon^{4}}-\frac{1}{\epsilon^{3}} H_{1}+\frac{1}{\epsilon^{2}}\left(2 H_{1,1}+\zeta_{2}\right)+\frac{1}{\epsilon}\left(\frac{1}{2} \zeta_{3}-H_{2,1}-\frac{3}{2} H_{1,2}-\frac{9}{2} H_{1,1,1}\right.\right. \\
& \left.-\frac{3}{2} \zeta_{2} H_{1}-2 H_{3}\right)+3 \zeta_{2} H_{1,1}+2 H_{3,1}-2 H_{2,2}+\frac{5}{2} H_{2,1,1}-3 H_{1,3} \\
& +3 H_{1,2,1}+H_{1,1,2}+\frac{19}{2} H_{1,1,1,1}+\frac{29 \zeta_{4}}{4}+\zeta_{2} H_{2}+3 \zeta_{3} H_{1}-8 H_{4} \\
& +\epsilon\left(-\frac{9}{2} \zeta_{2} H_{2,1}-\frac{9}{2} \zeta_{2} H_{1,2}-7 \zeta_{2} H_{1,1,1}-\frac{21}{2} \zeta_{3} H_{1,1}+8 H_{4,1}-7 H_{3,2}\right. \\
& -\frac{7}{2} H_{3,1,1}-7 H_{2,3}+\frac{5}{2} H_{2,2,1}+\frac{1}{2} H_{2,1,2}-\frac{11}{2} H_{2,1,1,1}-12 H_{1,4}+3 H_{1,3,1} \\
& -\frac{9}{2} H_{1,2,2}-\frac{15}{2} H_{1,2,1,1}+H_{1,1,3}-\frac{5}{2} H_{1,1,2,1}-3 H_{1,1,1,2}-\frac{39}{2} H_{1,1,1,1,1} \\
& \left.\left.-\frac{37}{2} \zeta_{5}+4 \zeta_{2} \zeta_{3}-4 \zeta_{2} H_{3}+\frac{7}{2} \zeta_{3} H_{2}+\frac{17}{8} \zeta_{4} H_{1}-32 H_{5}\right)\right] \\
& +\frac{1}{\epsilon}\left(-2 H_{2,1}-\frac{3}{2} H_{1,2}-\frac{3}{2} H_{1,1,1}+\frac{1}{2} \zeta_{2} H_{1}-2 H_{3}\right)-7 H_{3,1}-10 H_{2,2} \\
& -\frac{3}{2} H_{2,1,1}-7 H_{1,3}-3 H_{1,2,1}-6 H_{1,1,2}+\frac{3}{2} H_{1,1,1,1}+\zeta_{2} H_{2}+2 \zeta_{3} H_{1}-12 H_{4} \\
& +\epsilon\left(-\frac{13}{2} \zeta_{2} H_{2,1}-\frac{11}{2} \zeta_{2} H_{1,2}-3 \zeta_{2} H_{1,1,1}-\frac{1}{2} \zeta_{3} H_{1,1}-29 H_{4,1}-52 H_{3,2}\right. \\
& -\frac{19}{2} H_{3,1,1}-43 H_{2,3}-\frac{7}{2} H_{2,2,1}-\frac{47}{2} H_{2,1,2}-\frac{13}{2} H_{2,1,1,1}-28 H_{1,4}-14 H_{1,3,1} \\
& -\frac{55}{2} H_{1,2,2}-\frac{9}{2} H_{1,2,1,1}-25 H_{1,1,3}+\frac{7}{2} H_{1,1,2,1}-7 H_{1,1,1,2}-\frac{15}{2} H_{1,1,1,1,1}
\end{aligned}
$$




$$
\begin{aligned}
& \left.-7 \zeta_{2} H_{3}+\frac{7}{2} \zeta_{3} H_{2}+\frac{109}{8} \zeta_{4} H_{1}-56 H_{5}\right) \\
& +\frac{1}{N^{2}}\left[-\frac{1}{\epsilon}\left(H_{2,1}+H_{1,1,1}\right)-7 H_{3,1}-5 H_{2,2}-3 H_{2,1,1}-2 H_{1,3}-5 H_{1,2,1}\right. \\
& -6 H_{1,1,2}-\zeta_{2} H_{2}+\zeta_{3} H_{1}+\epsilon\left(-2 \zeta_{2} H_{2,1}-2 \zeta_{2} H_{1,2}-\zeta_{2} H_{1,1,1}-2 \zeta_{3} H_{1,1}\right. \\
& -37 H_{4,1}-35 H_{3,2}-9 H_{3,1,1}-26 H_{2,3}-10 H_{2,2,1}-24 H_{2,1,2}-4 H_{2,1,1,1} \\
& -8 H_{1,4}-19 H_{1,3,1}-24 H_{1,2,2}-H_{1,2,1,1}-24 H_{1,1,3}+4 H_{1,1,2,1}-7 H_{1,1,1,2} \\
& \left.\left.-4 H_{1,1,1,1,1}-7 \zeta_{2} H_{3}-2 \zeta_{3} H_{2}+\frac{11}{4} \zeta_{4} H_{1}\right)\right]+\mathcal{O}\left(\epsilon^{2}\right) \text {, } \\
& U_{g q}^{(2,1)}(z, \epsilon)=N^{2}\left[\frac{1}{\epsilon^{3}} H_{1}-\frac{1}{\epsilon^{2}}\left(-3 H_{1,1}-H_{2}\right)+\frac{1}{\epsilon}\left(3 H_{2,1}+2 H_{1,2}+7 H_{1,1,1}+\zeta_{2} H_{1}+H_{3}\right)\right. \\
& -3 \zeta_{2} H_{1,1}-3 H_{3,1}-2 H_{2,2}-7 H_{2,1,1}-2 H_{1,3}-6 H_{1,2,1}-4 H_{1,1,2} \\
& -15 H_{1,1,1,1}-\zeta_{2} H_{2}-6 \zeta_{3} H_{1}-H_{4}+\epsilon\left(3 \zeta_{2} H_{2,1}+2 \zeta_{2} H_{1,2}+7 \zeta_{2} H_{1,1,1}\right. \\
& +18 \zeta_{3} H_{1,1}+3 H_{4,1}+2 H_{3,2}+7 H_{3,1,1}+2 H_{2,3}+6 H_{2,2,1}+4 H_{2,1,2} \\
& +15 H_{2,1,1,1}+2 H_{1,4}+6 H_{1,3,1}+4 H_{1,2,2}+14 H_{1,2,1,1}+4 H_{1,1,3}+12 H_{1,1,2,1} \\
& \left.\left.+8 H_{1,1,1,2}+31 H_{1,1,1,1,1}+\zeta_{2} H_{3}+6 \zeta_{3} H_{2}-\frac{29}{4} \zeta_{4} H_{1}+H_{5}\right)\right] \\
& -\frac{1}{\epsilon^{3}} H_{1}+\frac{1}{\epsilon^{2}}\left(H_{1,1}-H_{2}\right)+\frac{1}{\epsilon}\left(H_{2,1}-H_{1,1,1}-\zeta_{2} H_{1}-H_{3}\right)+\zeta_{2} H_{1,1}+H_{3,1} \\
& -H_{2,1,1}+H_{1,1,1,1}-\zeta_{2} H_{2}+6 \zeta_{3} H_{1}-H_{4}+\epsilon\left(\zeta_{2} H_{2,1}-\zeta_{2} H_{1,1,1}-6 \zeta_{3} H_{1,1}\right. \\
& \left.+H_{4,1}-H_{3,1,1}+H_{2,1,1,1}-H_{1,1,1,1,1}-\zeta_{2} H_{3}+6 \zeta_{3} H_{2}+\frac{29}{4} \zeta_{4} H_{1}-H_{5}\right)+\mathcal{O}\left(\epsilon^{2}\right), \\
& U_{g q}^{(2,0)}(z, \epsilon)=N^{2}\left[\frac{1}{\epsilon^{2}} H_{1,1}+\frac{1}{\epsilon}\left(-H_{2,1}+\frac{1}{2} H_{1,2}-\frac{5}{2} H_{1,1,1}+\frac{1}{2} \zeta_{2} H_{1}+2 H_{3}\right)-2 H_{3,1}\right. \\
& -H_{2,2}+\frac{5}{2} H_{2,1,1}-2 H_{1,3}+H_{1,2,1}+H_{1,1,2}+\frac{11}{2} H_{1,1,1,1}+8 \zeta_{3} H_{1}-4 H_{4} \\
& +\epsilon\left(-\frac{1}{2} \zeta_{2} H_{2,1}-\frac{3}{2} \zeta_{2} H_{1,2}-\frac{21}{2} \zeta_{3} H_{1,1}+8 H_{4,1}+13 H_{3,2}+\frac{7}{2} H_{3,1,1}\right. \\
& +7 H_{2,3}+\frac{1}{2} H_{2,2,1}+\frac{5}{2} H_{2,1,2}-\frac{11}{2} H_{2,1,1,1}+11 H_{1,4}+2 H_{1,3,1}+\frac{19}{2} H_{1,2,2} \\
& -\frac{5}{2} H_{1,2,1,1}+2 H_{1,1,3}-\frac{11}{2} H_{1,1,2,1}-H_{1,1,1,2}-\frac{23}{2} H_{1,1,1,1,1}-2 \zeta_{2} H_{3} \\
& \left.\left.-\frac{21}{2} \zeta_{3} H_{2}+\frac{75}{8} \zeta_{4} H_{1}+16 H_{5}\right)\right] \\
& -\frac{2}{\epsilon^{2}} H_{1,1}+\frac{1}{\epsilon}\left(2 H_{2,1}+\frac{3}{2} H_{1,2}+\frac{3}{2} H_{1,1,1}-\frac{3}{2} \zeta_{2} H_{1}+2 H_{3}\right)-\zeta_{2} H_{1,1}-H_{3,1} \\
& +4 H_{2,2}-\frac{3}{2} H_{2,1,1}-2 H_{1,2,1}+3 H_{1,1,2}-\frac{7}{2} H_{1,1,1,1}-6 \zeta_{3} H_{1}+\epsilon\left(-\frac{1}{2} \zeta_{2} H_{2,1}\right. \\
& +\frac{7}{2} \zeta_{2} H_{1,2}-\frac{5}{2} \zeta_{3} H_{1,1}+11 H_{4,1}+21 H_{3,2}+\frac{19}{2} H_{3,1,1}+13 H_{2,3}+\frac{5}{2} H_{2,2,1} \\
& +\frac{25}{2} H_{2,1,2}+\frac{19}{2} H_{2,1,1,1}+2 H_{1,4}+10 H_{1,3,1}+\frac{25}{2} H_{1,2,2}+\frac{15}{2} H_{1,2,1,1}
\end{aligned}
$$




$$
\begin{aligned}
& +11 H_{1,1,3}+\frac{7}{2} H_{1,1,2,1}+6 H_{1,1,1,2}+\frac{15}{2} H_{1,1,1,1,1}+\zeta_{2} H_{3}-\frac{9}{2} \zeta_{3} H_{2} \\
& \left.-\frac{45}{8} \zeta_{4} H_{1}+8 H_{5}\right) \\
& +\frac{1}{N^{2}}\left[\frac{1}{\epsilon^{2}} H_{1,1}+\frac{1}{\epsilon}\left(3 H_{2,1}+H_{1,2}+4 H_{1,1,1}\right)+7 H_{3,1}+6 H_{2,2}+7 H_{2,1,1}\right. \\
& +4 H_{1,3}+8 H_{1,2,1}+9 H_{1,1,2}+7 H_{1,1,1,1}+\zeta_{2} H_{2}-3 \zeta_{3} H_{1}+\epsilon\left(2 \zeta_{2} H_{1,2}\right. \\
& -3 \zeta_{2} H_{1,1,1}+6 \zeta_{3} H_{1,1}+15 H_{4,1}+16 H_{3,2}+13 H_{3,1,1}+14 H_{2,3} \\
& +10 H_{2,2,1}+18 H_{2,1,2}+18 H_{2,1,1,1}-3 H_{1,4}+14 H_{1,3,1}+14 H_{1,2,2}+14 H_{1,2,1,1} \\
& +15 H_{1,1,3}+11 H_{1,1,2,1}+18 H_{1,1,1,2}+19 H_{1,1,1,1,1}+3 \zeta_{2} H_{3}+4 \zeta_{3} H_{2} \\
& \left.\left.-\frac{49}{4} \zeta_{4} H_{1}\right)\right]+\mathcal{O}\left(\epsilon^{2}\right) \\
& U_{q q}^{(2,2)}(z, \epsilon)=N^{2}\left[\frac{1}{2 \epsilon^{4}}-\frac{1}{\epsilon^{3}} H_{1}+\frac{1}{\epsilon^{2}}\left(2 H_{1,1}+\zeta_{2}\right)+\frac{1}{\epsilon}\left(\frac{1}{2} H_{2,1}+H_{1,2}-\frac{7}{2} H_{1,1,1}-\zeta_{3}\right.\right. \\
& \left.-2 \zeta_{2} H_{1}+H_{3}\right)+\frac{5}{2} \zeta_{2} H_{1,1}+H_{3,1}+\frac{11}{2} H_{2,2}+\frac{1}{2} H_{2,1,1}+4 H_{1,3} \\
& -H_{1,2,1}+\frac{3}{2} H_{1,1,2}+\frac{13}{2} H_{1,1,1,1}+\frac{35 \zeta_{4}}{8}-\frac{3}{2} \zeta_{2} H_{2}+5 \zeta_{3} H_{1}+8 H_{4} \\
& +\epsilon\left(\frac{3}{2} \zeta_{2} H_{2,1}+2 \zeta_{2} H_{1,2}-\frac{5}{2} \zeta_{2} H_{1,1,1}-\frac{35}{2} \zeta_{3} H_{1,1}-4 H_{4,1}\right. \\
& +18 H_{3,2}+H_{3,1,1}+18 H_{2,3}-\frac{15}{2} H_{2,2,1}+\frac{1}{2} H_{2,1,2}-\frac{5}{2} H_{2,1,1,1}+16 H_{1,4} \\
& -4 H_{1,3,1}+2 H_{1,2,2}+H_{1,2,1,1}+6 H_{1,1,3}-\frac{3}{2} H_{1,1,2,1}-\frac{7}{2} H_{1,1,1,2}-\frac{25}{2} H_{1,1,1,1,1} \\
& \left.\left.-\frac{57}{2} \zeta_{5}-\frac{1}{2} \zeta_{2} \zeta_{3}-2 \zeta_{2} H_{3}-\frac{3}{2} \zeta_{3} H_{2}-3 \zeta_{4} H_{1}+40 H_{5}\right)\right] \\
& +\frac{1}{\epsilon}\left(\frac{1}{2} H_{2,1}+H_{1,2}+\frac{1}{2} H_{1,1,1}-\frac{3 \zeta_{3}}{2}+H_{3}\right)-\frac{1}{2} \zeta_{2} H_{1,1}-H_{3,1}+\frac{5}{2} H_{2,2} \\
& -\frac{1}{2} H_{2,1,1}+2 H_{1,3}-2 H_{1,2,1}+\frac{1}{2} H_{1,1,2}-\frac{3}{2} H_{1,1,1,1}-\frac{23 \zeta_{4}}{8}-\frac{1}{2} \zeta_{2} H_{2}+3 \zeta_{3} H_{1} \\
& +4 H_{4}+\epsilon\left(\frac{3}{2} \zeta_{2} H_{2,1}+3 \zeta_{2} H_{1,2}+\frac{5}{2} \zeta_{2} H_{1,1,1}-\frac{11}{2} \zeta_{3} H_{1,1}-4 H_{4,1}+8 H_{3,2}\right. \\
& +4 H_{3,1,1}+8 H_{2,3}-\frac{7}{2} H_{2,2,1}+\frac{1}{2} H_{2,1,2}+\frac{1}{2} H_{2,1,1,1}+8 H_{1,4}-2 H_{1,3,1} \\
& +3 H_{1,2,2}+5 H_{1,2,1,1}+4 H_{1,1,3}+\frac{1}{2} H_{1,1,2,1}-\frac{1}{2} H_{1,1,1,2}+\frac{7}{2} H_{1,1,1,1,1}-10 \zeta_{5} \\
& \left.-\frac{9}{2} \zeta_{2} \zeta_{3}+2 \zeta_{2} H_{3}+\frac{1}{2} \zeta_{3} H_{2}+\frac{23}{4} \zeta_{4} H_{1}+16 H_{5}\right)+\mathcal{O}\left(\epsilon^{2}\right), \\
& U_{q q}^{(2,1)}(z, \epsilon)=N^{2}\left[-\frac{1}{\epsilon^{4}}+\frac{3}{\epsilon^{3}} H_{1}-\frac{1}{\epsilon^{2}}\left(5 H_{1,1}+\zeta_{2}\right)+\frac{1}{\epsilon}\left(2 H_{2,1}+2 H_{1,2}+9 H_{1,1,1}+6 \zeta_{3}\right.\right. \\
& \left.+3 \zeta_{2} H_{1}+2 H_{3}\right)-5 \zeta_{2} H_{1,1}-4 H_{3,1}-2 H_{2,2}-6 H_{2,1,1}-2 H_{1,3} \\
& -6 H_{1,2,1}-4 H_{1,1,2}-17 H_{1,1,1,1}+\frac{29 \zeta_{4}}{4}-18 \zeta_{3} H_{1}+\epsilon\left(2 \zeta_{2} H_{2,1}+2 \zeta_{2} H_{1,2}\right.
\end{aligned}
$$




$$
\begin{aligned}
& +9 \zeta_{2} H_{1,1,1}+30 \zeta_{3} H_{1,1}+2 H_{4,1}+2 H_{3,2}+8 H_{3,1,1}+2 H_{2,3}+6 H_{2,2,1} \\
& +4 H_{2,1,2}+14 H_{2,1,1,1}+2 H_{1,4}+6 H_{1,3,1}+4 H_{1,2,2}+14 H_{1,2,1,1}+4 H_{1,1,3} \\
& \left.+12 H_{1,1,2,1}+8 H_{1,1,1,2}+33 H_{1,1,1,1,1}+42 \zeta_{5}+6 \zeta_{2} \zeta_{3}+2 \zeta_{2} H_{3}-\frac{87}{4} \zeta_{4} H_{1}+2 H_{5}\right) \\
& -\frac{1}{\epsilon^{4}}+\frac{1}{\epsilon^{3}} H_{1}-\frac{1}{\epsilon^{2}}\left(H_{1,1}+\zeta_{2}\right)+\frac{1}{\epsilon}\left(H_{1,1,1}+6 \zeta_{3}+\zeta_{2} H_{1}\right)-\zeta_{2} H_{1,1}-H_{1,1,1,1} \\
& +\frac{29}{4} \zeta_{4}-6 \zeta_{3} H_{1}+\epsilon\left(\zeta_{2} H_{1,1,1}+6 \zeta_{3} H_{1,1}+H_{1,1,1,1,1}+42 \zeta_{5}+6 \zeta_{2} \zeta_{3}\right. \\
& \left.-\frac{29}{4} \zeta_{4} H_{1}\right)+\mathcal{O}\left(\epsilon^{2}\right) \\
& U_{q q}^{(2,0)}(z, \epsilon)=N^{2}\left[\frac{1}{2 \epsilon^{4}}-\frac{2}{\epsilon^{3}} H_{1}+\frac{1}{\epsilon^{2}}\left(4 H_{1,1}-\frac{1}{2} \zeta_{2}\right)+\frac{1}{\epsilon}\left(-\frac{5}{2} H_{2,1}-3 H_{1,2}-\frac{5}{2} H_{1,1,1}\right.\right. \\
& \left.-2 \zeta_{3}+2 \zeta_{2} H_{1}-3 H_{3}\right)+\frac{5}{2} \zeta_{2} H_{1,1}+9 H_{3,1}-\frac{1}{2} H_{2,2}+\frac{25}{2} H_{2,1,1} \\
& +3 H_{1,3}+14 H_{1,2,1}+\frac{11}{2} H_{1,1,2}+\frac{35}{2} H_{1,1,1,1}+\frac{43 \zeta_{4}}{8}+\frac{5}{2} \zeta_{2} H_{2}+11 \zeta_{3} H_{1} \\
& -3 H_{4}+\epsilon\left(\frac{9}{2} \zeta_{2} H_{2,1}-\zeta_{2} H_{1,2}+\frac{3}{2} \zeta_{2} H_{1,1,1}-\frac{7}{2} \zeta_{3} H_{1,1}-3 H_{3,2}-3 H_{3,1,1}\right. \\
& -4 H_{2,3}-\frac{3}{2} H_{2,2,1}-\frac{1}{2} H_{2,1,2}-\frac{5}{2} H_{2,1,1,1}-7 H_{1,4}-6 H_{1,3,1}-6 H_{1,2,1,1} \\
& -6 H_{1,1,3}-\frac{15}{2} H_{1,1,2,1}+\frac{5}{2} H_{1,1,1,2}-\frac{11}{2} H_{1,1,1,1,1}+34 \zeta_{5}+\frac{1}{2} \zeta_{2} \zeta_{3}+2 \zeta_{2} H_{3} \\
& \left.-\frac{7}{2} \zeta_{3} H_{2}+\frac{11}{4} \zeta_{4} H_{1}-5 H_{5}\right) \\
& +\frac{1}{\epsilon^{4}}-\frac{2}{\epsilon^{3}} H_{1}+\frac{1}{2 \epsilon^{2}} \zeta_{2}+\frac{1}{\epsilon}\left(-\frac{5}{2} H_{2,1}-3 H_{1,2}-\frac{5}{2} H_{1,1,1}-5 \zeta_{3}-3 H_{3}\right) \\
& +\frac{3}{2} \zeta_{2} H_{1,1}+3 H_{3,1}-\frac{3}{2} H_{2,2}+\frac{3}{2} H_{2,1,1}+H_{1,3}+3 H_{1,2,1}-\frac{3}{2} H_{1,1,2}+\frac{3}{2} H_{1,1,1,1} \\
& -\frac{1}{8} \zeta_{4}+\frac{3}{2} \zeta_{2} H_{2}+H_{4}+\epsilon\left(\frac{9}{2} \zeta_{2} H_{2,1}+2 \zeta_{2} H_{1,2}+\frac{9}{2} \zeta_{2} H_{1,1,1}-\frac{3}{2} \zeta_{3} H_{1,1}\right. \\
& \text { - } 12 H_{4,1}-11 H_{3,2}-10 H_{3,1,1}-12 H_{2,3}-\frac{19}{2} H_{2,2,1}-\frac{17}{2} H_{2,1,2}-\frac{11}{2} H_{2,1,1,1} \\
& -13 H_{1,4}-12 H_{1,3,1}-11 H_{1,2,2}-10 H_{1,2,1,1}-12 H_{1,1,3}-\frac{19}{2} H_{1,1,2,1} \\
& \left.-\frac{17}{2} H_{1,1,1,2}-\frac{11}{2} H_{1,1,1,1,1}+31 \zeta_{5}+\frac{9}{2} \zeta_{2} \zeta_{3}+2 \zeta_{2} H_{3}-\frac{3}{2} \zeta_{3} H_{2}-13 H_{5}\right) \\
& +\frac{1}{N^{2}}\left[\frac{1}{2 \epsilon^{4}}-\frac{3}{\epsilon} \zeta_{3}-\frac{11}{2} \zeta_{4}+\epsilon\left(4 \zeta_{2} \zeta_{3}-3 \zeta_{5}\right)\right]+\mathcal{O}\left(\epsilon^{2}\right) \text {. }
\end{aligned}
$$

The functions $V_{i j}^{(2, k)}(z)$ are not of maximal weight, and are given by

$$
\begin{aligned}
V_{g g}^{(2,2)}(z, \epsilon)= & N^{3}\left[\frac { 1 } { \epsilon } \left(\frac{12 z^{3}-33 z^{2}+32 z-22}{6(1-z) z} H_{2}-\frac{1}{3}(1-z) H_{1}\right.\right. \\
& \left.-\frac{44 z^{4}-76 z^{3}+99 z^{2}-56 z+22}{6(1-z) z} \zeta_{2}-\frac{404 z^{4}-799 z^{3}+1185 z^{2}-772 z+386}{54(1-z) z}\right) \\
& +\frac{(z+1)\left(11 z^{2}-10 z+11\right)}{3 z} H_{2,1}-\frac{44 z^{3}-100 z^{2}+105 z-60}{6(1-z)} H_{1,2}
\end{aligned}
$$




$$
\begin{aligned}
& +\frac{22 z^{3}-34 z^{2}+35 z-11}{6 z} H_{1,1,1}+\frac{5-6 z}{6} H_{1,1} \\
& +\frac{72 z^{3}-201 z^{2}+196 z-134}{18(1-z) z} H_{2}+\frac{88 z^{4}-164 z^{3}+231 z^{2}-144 z+66}{6(1-z) z} \zeta_{2} H_{1} \\
& +\frac{404 z^{4}-823 z^{3}+1224 z^{2}-787 z+386}{27(1-z) z} H_{1}-\frac{22 z^{4}-56 z^{3}+99 z^{2}-76 z+44}{3(1-z) z} H_{3} \\
& -\frac{268 z^{4}-464 z^{3}+597 z^{2}-334 z+134}{18(1-z) z} \zeta_{2}+\frac{66 z^{4}-144 z^{3}+231 z^{2}-164 z+88}{3(1-z) z} \zeta_{3} \\
& -\frac{2428 z^{4}-4757 z^{3}+7041 z^{2}-4568 z+2284}{162(1-z) z} \\
& +\epsilon\left(-\frac{14576 z^{4}-28387 z^{3}+42027 z^{2}-27280 z+13640}{486(1-z) z}\right. \\
& +\frac{2428 z^{4}-4913 z^{3}+7308 z^{2}-4733 z+2338}{81(1-z) z} H_{1} \\
& +\frac{405 z^{3}-1221 z^{2}+1292 z-880}{54(1-z) z} H_{2} \\
& -\frac{1616 z^{4}-3322 z^{3}+4941 z^{2}-3163 z+1544}{54(1-z) z} H_{1,1} \\
& -\frac{134 z^{4}-334 z^{3}+600 z^{2}-467 z+268}{9(1-z) z} H_{3}+\frac{134 z^{3}+16 z^{2}+13 z+134}{18 z} H_{2,1} \\
& -\frac{268 z^{3}-572 z^{2}+546 z-309}{18(1-z)} H_{1,2}+\frac{134 z^{3}-146 z^{2}+157 z-67}{18 z} H_{1,1,1} \\
& -4 \frac{22 z^{4}-56 z^{3}+99 z^{2}-76 z+44}{3(1-z) z} H_{4}+\frac{22 z^{4}-56 z^{3}+99 z^{2}-76 z+44}{3(1-z) z} H_{3,1} \\
& -\frac{110 z^{4}-328 z^{3}+447 z^{2}-328 z+110}{6(1-z) z} H_{2,2} \\
& +\frac{22 z^{4}+52 z^{3}-108 z^{2}+122 z-55}{6(1-z) z} H_{2,1,1} \\
& -\frac{44 z^{4}-112 z^{3}+108 z^{2}-62 z-11}{3(1-z) z} H_{1,3}-\frac{88 z^{3}-100 z^{2}+107 z-11}{6 z} H_{1,2,1} \\
& -\frac{22 z^{4}-68 z^{3}+102 z^{2}-78 z+33}{6(1-z) z} H_{1,1,2}-\frac{22 z^{3}-34 z^{2}+35 z-11}{2 z} H_{1,1,1,1} \\
& -\frac{1616 z^{4}-2755 z^{3}+3453 z^{2}-1766 z+664}{54(1-z) z} \zeta_{2} \\
& +\frac{22 z^{4}-8 z^{3}-33 z^{2}+52 z-44}{6(1-z) z} \zeta_{2} H_{2} \\
& +\frac{536 z^{4}-1024 z^{3}+1446 z^{2}-891 z+402}{18(1-z) z} \zeta_{2} H_{1} \\
& -\frac{154 z^{4}-284 z^{3}+426 z^{2}-274 z+143}{6(1-z) z} \zeta_{2} H_{1,1} \\
& +\frac{804 z^{4}-1734 z^{3}+2835 z^{2}-2039 z+1072}{18(1-z) z} \zeta_{3} \\
& -\frac{264 z^{4}-588 z^{3}+867 z^{2}-598 z+275}{6(1-z) z} \zeta_{3} H_{1} \\
& \left.\left.-\frac{110 z^{4}+128 z^{3}-627 z^{2}+708 z-528}{8(1-z) z} \zeta_{4}\right)\right] \\
& +N N_{f}\left[\frac { 1 } { \epsilon } \left(-\frac{3 z^{3}-6 z^{2}+5 z-4}{6(1-z) z} H_{2}+\frac{1}{3}(1-z) H_{1}\right.\right.
\end{aligned}
$$




$$
\begin{aligned}
& \left.+\frac{8 z^{4}-13 z^{3}+18 z^{2}-11 z+4}{6(1-z) z} \zeta_{2}+\frac{56 z^{4}-103 z^{3}+141 z^{2}-76 z+38}{54(1-z) z}\right) \\
& -\frac{(z+1)\left(2 z^{2}-z+2\right)}{3 z} H_{2,1}+\frac{8 z^{3}-19 z^{2}+24 z-15}{6(1-z)} H_{1,2} \\
& -\frac{4 z^{3}-7 z^{2}+8 z-2}{6 z} H_{1,1,1}+\frac{6 z-5}{6} H_{1,1}-\frac{9 z^{3}-15 z^{2}+11 z-10}{9(1-z) z} H_{2} \\
& -\frac{16 z^{4}-29 z^{3}+42 z^{2}-27 z+12}{6(1-z) z} \zeta_{2} H_{1}-2 \frac{28 z^{4}-68 z^{3}+99 z^{2}-50 z+19}{27(1-z) z} H_{1} \\
& +\frac{4 z^{4}-11 z^{3}+18 z^{2}-13 z+8}{3(1-z) z} H_{3}+\frac{20 z^{4}-31 z^{3}+42 z^{2}-26 z+10}{9(1-z) z} \zeta_{2} \\
& -\frac{12 z^{4}-27 z^{3}+42 z^{2}-29 z+16}{3(1-z) z} \zeta_{3}+\frac{164 z^{4}-265 z^{3}+330 z^{2}-130 z+65}{81(1-z) z} \\
& +\epsilon\left(\frac{1952 z^{4}-2761 z^{3}+3183 z^{2}-844 z+422}{486(1-z) z}\right. \\
& -\frac{328 z^{4}-785 z^{3}+1098 z^{2}-497 z+184}{81(1-z) z} H_{1}-\frac{81 z^{3}-177 z^{2}+224 z-184}{54(1-z) z} H_{2} \\
& +4 \frac{28 z^{4}-74 z^{3}+108 z^{2}-53 z+19}{27(1-z) z} H_{1,1}+\frac{20 z^{4}-52 z^{3}+87 z^{2}-65 z+40}{9(1-z) z} H_{3} \\
& -\frac{20 z^{3}+22 z^{2}+19 z+20}{18 z} H_{2,1}+\frac{40 z^{3}-62 z^{2}+51 z-39}{18(1-z)} H_{1,2} \\
& -\frac{(z+1)\left(10 z^{2}+z-5\right)}{9 z} H_{1,1,1}+4 \frac{4 z^{4}-11 z^{3}+18 z^{2}-13 z+8}{3(1-z) z} H_{4} \\
& -\frac{4 z^{4}-11 z^{3}+18 z^{2}-13 z+8}{3(1-z) z} H_{3,1}+\frac{\left(4 z^{2}-7 z+4\right)\left(5 z^{2}-8 z+5\right)}{6(1-z) z} H_{2,2} \\
& -\frac{4 z^{4}+16 z^{3}-27 z^{2}+23 z-10}{6(1-z) z} H_{2,1,1}+\frac{8 z^{4}-22 z^{3}+27 z^{2}-17 z-2}{3(1-z) z} H_{1,3} \\
& +\frac{16 z^{3}-19 z^{2}+26 z-2}{6 z} H_{1,2,1}+\frac{4 z^{4}-14 z^{3}+21 z^{2}-15 z+6}{6(1-z) z} H_{1,1,2} \\
& +\frac{4 z^{3}-7 z^{2}+8 z-2}{2 z} H_{1,1,1,1}+\frac{224 z^{4}-295 z^{3}+303 z^{2}-32 z-32}{54(1-z) z} \zeta_{2} \\
& -\frac{80 z^{4}-166 z^{3}+249 z^{2}-153 z+60}{18(1-z) z} \zeta_{2} H_{1}-\frac{4 z^{4}+z^{3}-6 z^{2}+7 z-8}{6(1-z) z} \zeta_{2} H_{2} \\
& +\frac{28 z^{4}-50 z^{3}+75 z^{2}-49 z+26}{6(1-z) z} \zeta_{2} H_{1,1} \\
& -\frac{120 z^{4}-258 z^{3}+441 z^{2}-323 z+160}{18(1-z) z} \zeta_{3} \\
& +\frac{48 z^{4}-111 z^{3}+165 z^{2}-112 z+50}{6(1-z) z} \zeta_{3} H_{1} \\
& \left.\left.+\frac{20 z^{4}+47 z^{3}-114 z^{2}+105 z-96}{8(1-z) z} \zeta_{4}\right)\right]+\mathcal{O}\left(\epsilon^{2}\right) \\
& V_{g g}^{(2,1)}(z, \epsilon)=2 N^{2}\left(N-N_{f}\right) \epsilon \zeta_{3}+\mathcal{O}\left(\epsilon^{2}\right), \\
& V_{g g}^{(2,1)}(z, \epsilon)=N^{3}\left[\frac { 1 } { \epsilon } \left(-\frac{12 z^{3}-33 z^{2}+32 z-22}{6(1-z) z} H_{2}\right.\right. \\
& -\frac{134 z^{4}-271 z^{3}+402 z^{2}-265 z+134}{9(1-z) z} H_{1}+\frac{22 z^{3}-32 z^{2}+33 z-12}{6(1-z)} \zeta_{2} \\
& \left.+\frac{6 z^{3}-9 z^{2}-13 z+22}{18(1-z)}\right)-\frac{44 z^{4}-76 z^{3}+139 z^{2}-96 z+66}{2(1-z) z} H_{1,2}
\end{aligned}
$$




$$
\begin{aligned}
& -\frac{77 z^{4}-166 z^{3}+264 z^{2}-186 z+99}{3(1-z) z} H_{2,1} \\
& -\frac{154 z^{4}-296 z^{3}+459 z^{2}-306 z+165}{6(1-z) z} H_{1,1,1}-\frac{5-6 z}{6} H_{1,1} \\
& +\frac{12 z^{3}+7 z^{2}-8 z+22}{2(1-z) z} \zeta_{2} H_{1}-\frac{96 z^{3}-237 z^{2}+208 z-134}{18(1-z) z} H_{2} \\
& -\frac{66 z^{4}-144 z^{3}+231 z^{2}-164 z+88}{3(1-z) z} H_{3} \\
& -\frac{2\left(386 z^{4}-784 z^{3}+1167 z^{2}-769 z+395\right)}{27(1-z) z} H_{1}+\frac{134 z^{3}-184 z^{2}+195 z-78}{18(1-z)} \zeta_{2} \\
& -\frac{22 z^{3}-32 z^{2}+33 z-12}{3(1-z)} \zeta_{3}-\frac{84 z^{3}-201 z^{2}+715 z-646}{54(1-z)} \\
& +\epsilon\left(-\frac{1188 z^{3}-2461 z^{2}+4884 z-3715}{54(1-z)}-\frac{18 z^{3}-31 z^{2}-35 z+24}{18(1-z) z} H_{1,1}\right. \\
& +\frac{22 z^{3}-34 z^{2}+35 z-11}{2 z} H_{1,1,1,1}-\frac{22 z^{3}-32 z^{2}+33 z-12}{1-z} H_{3,1} \\
& -\frac{525 z^{3}-1401 z^{2}+1208 z-808}{54(1-z) z} H_{2}+\frac{22 z^{4}-80 z^{3}+125 z^{2}-100 z+44}{2(1-z) z} H_{2,2} \\
& -\frac{22 z^{4}-20 z^{3}+10 z^{2}+10 z-11}{2(1-z) z} H_{2,1,1}-\frac{22 z^{4}-20 z^{3}+30 z^{2}-10 z+11}{3(1-z) z} H_{1,3} \\
& -\frac{22 z^{4}-8 z^{3}-33 z^{2}+52 z-44}{3(1-z) z} H_{4}+\frac{66 z^{4}-180 z^{3}+240 z^{2}-170 z+55}{6(1-z) z} H_{1,1,2} \\
& -\frac{132 z^{4}-252 z^{3}+333 z^{2}-202 z+77}{6(1-z) z} H_{1,2,1} \\
& -\frac{268 z^{4}-480 z^{3}+890 z^{2}-611 z+402}{6(1-z) z} H_{1,2} \\
& -\frac{402 z^{4}-876 z^{3}+1407 z^{2}-1000 z+536}{9(1-z) z} H_{3} \\
& -\frac{938 z^{4}-2038 z^{3}+3249 z^{2}-2283 z+1206}{18(1-z) z} H_{2,1} \\
& -\frac{938 z^{4}-1864 z^{3}+2901 z^{2}-1908 z+1005}{18(1-z) z} H_{1,1,1} \\
& -2 \frac{2230 z^{4}-4538 z^{3}+6870 z^{2}-4589 z+2383}{81(1-z) z} H_{1} \\
& -\frac{22 z^{3}-56 z^{2}+59 z-36}{2(1-z)} \zeta_{2} H_{2}+\frac{76 z^{3}+52 z^{2}-61 z+134}{6(1-z) z} \zeta_{2} H_{1} \\
& -\frac{66 z^{4}-228 z^{3}+252 z^{2}-178 z+11}{6(1-z) z} \zeta_{2} H_{1,1} \\
& +\frac{264 z^{4}-540 z^{3}+775 z^{2}-510 z+231}{2(1-z) z} \zeta_{3} H_{1} \\
& +\frac{952 z^{3}-1367 z^{2}+1605 z-714}{54(1-z)} \zeta_{2}-\frac{268 z^{3}-386 z^{2}+417 z-165}{18(1-z)} \zeta_{3} \\
& \left.\left.+53 \frac{22 z^{3}-32 z^{2}+33 z-12}{24(1-z)} \zeta_{4}\right)\right] \\
& +N^{2} N_{f}\left[\frac { 1 } { \epsilon } \left(\frac{3 z^{3}-6 z^{2}+5 z-4}{6(1-z) z} H_{2}+\frac{20 z^{4}-43 z^{3}+60 z^{2}-37 z+20}{9(1-z) z} H_{1}\right.\right. \\
& \left.-\frac{4 z^{3}-5 z^{2}+6 z-3}{6(1-z)} \zeta_{2}-\frac{6 z^{3}-9 z^{2}-17 z+26}{18(1-z)}\right)
\end{aligned}
$$




$$
\begin{aligned}
& +\frac{\left(2 z^{2}-3 z+3\right)\left(7 z^{2}-5 z+6\right)}{3(1-z) z} H_{2,1}+\frac{8 z^{4}-13 z^{3}+22 z^{2}-15 z+12}{2(1-z) z} H_{1,2} \\
& +\frac{28 z^{4}-53 z^{3}+81 z^{2}-54 z+30}{6(1-z) z} H_{1,1,1}+\frac{5-6 z}{6} H_{1,1}-\frac{3 z^{3}-2 z^{2}+z+4}{2(1-z) z} \zeta_{2} H_{1} \\
& +\frac{21 z^{3}-33 z^{2}+17 z-10}{9(1-z) z} H_{2}+\frac{12 z^{4}-27 z^{3}+42 z^{2}-29 z+16}{3(1-z) z} H_{3} \\
& +\frac{76 z^{4}-185 z^{3}+246 z^{2}-137 z+94}{27(1-z) z} H_{1}-\frac{10 z^{3}-5 z^{2}+12 z-12}{9(1-z)} \zeta_{2} \\
& +\frac{4 z^{3}-5 z^{2}+6 z-3}{3(1-z)} \zeta_{3}+\frac{90 z^{3}-222 z^{2}+869 z-803}{54(1-z)} \\
& +\epsilon\left(\frac{2872 z^{3}-5998 z^{2}+12421 z-9635}{108(1-z)}-\frac{4 z^{3}-7 z^{2}+8 z-2}{2 z} H_{1,1,1,1}\right. \\
& +\frac{4 z^{3}-5 z^{2}+6 z-3}{1-z} H_{3,1}+2 \frac{9 z^{3}-16 z^{2}-5 z+6}{9(1-z) z} H_{1,1}+\frac{273 z^{3}-465 z^{2}+176 z-112}{54(1-z) z} H_{2} \\
& -\frac{4 z^{4}-17 z^{3}+26 z^{2}-19 z+8}{2(1-z) z} H_{2,2}+\frac{4 z^{4}-2 z^{3}+z^{2}+z-2}{2(1-z) z} H_{2,1,1} \\
& +\frac{4 z^{4}-2 z^{3}+3 z^{2}-z+2}{3(1-z) z} H_{1,3}+\frac{4 z^{4}+z^{3}-6 z^{2}+7 z-8}{3(1-z) z} H_{4} \\
& -\frac{12 z^{4}-36 z^{3}+51 z^{2}-35 z+10}{6(1-z) z} H_{1,1,2}+\frac{24 z^{4}-45 z^{3}+63 z^{2}-40 z+14}{6(1-z) z} H_{1,2,1} \\
& +\frac{2\left(30 z^{4}-69 z^{3}+105 z^{2}-71 z+40\right)}{9(1-z) z} H_{3}+\frac{40 z^{4}-78 z^{3}+137 z^{2}-89 z+60}{6(1-z) z} H_{1,2} \\
& +\frac{70 z^{4}-161 z^{3}+249 z^{2}-153 z+75}{9(1-z) z} H_{1,1,1}+\frac{140 z^{4}-334 z^{3}+513 z^{2}-339 z+180}{18(1-z) z} H_{2,1} \\
& +\frac{152 z^{4}-559 z^{3}+870 z^{2}-517 z+512}{81(1-z) z} H_{1}+\frac{\left(4 z^{3}-11 z^{2}+14 z-9\right) \zeta_{2}}{2(1-z)} H_{2} \\
& -\frac{22 z^{3}-17 z^{2}+5 z+20}{6(1-z) z} \zeta_{2} H_{1}+\frac{12 z^{4}-48 z^{3}+63 z^{2}-43 z+2}{6(1-z) z} \zeta_{2} H_{1,1} \\
& -\frac{48 z^{4}-99 z^{3}+145 z^{2}-96 z+42}{2(1-z) z} \zeta_{3} H_{1}-\frac{256 z^{3}-263 z^{2}+597 z-462}{54(1-z)} \zeta_{2} \\
& \left.\left.+\frac{40 z^{3}-38 z^{2}+111 z-93}{18(1-z)} \zeta_{3}-53 \frac{4 z^{3}-5 z^{2}+6 z-3}{24(1-z)} \zeta_{4}\right)\right] \\
& +N N_{f}^{2}\left[\frac{2}{9 \epsilon}+\frac{2}{27}\left(6 z^{2}-6 z+19\right)+\epsilon\left(\frac{2}{27}\left(38 z^{2}-38 z+75\right)\right)\right] \\
& +N_{f}\left[-\frac{1}{2}+\epsilon\left(\zeta_{2}-2 \zeta_{3}+\frac{1}{12}\left(-12 z^{2}+12 z-37\right)\right)\right]+\mathcal{O}\left(\epsilon^{2}\right), \\
& V_{g q}^{(2,2)}(z, \epsilon)=N^{3}\left[\frac { 1 } { \epsilon } \left(\frac{13\left(z^{2}-2 z+2\right)}{24 z} H_{1,1}+\frac{13 z^{2}-20 z+8}{24 z} H_{2}+\frac{z-2}{8 z} H_{1}\right.\right. \\
& \left.-\frac{31 z^{2}-56 z+44}{24 z} \zeta_{2}-\frac{202 z^{2}-395 z+386}{108 z}\right)+\frac{4 z^{2}-11 z+26}{12 z} H_{1,2} \\
& -\frac{11 z^{2}-16 z+4}{12 z} H_{2,1}-\frac{16 z^{2}-32 z+29}{8 z} H_{1,1,1}+\frac{32 z^{2}-151 z+178}{72 z} H_{1,1} \\
& +\frac{31 z^{2}-59 z+53}{12 z} \zeta_{2} H_{1}+\frac{16 z^{2}-23 z-10}{36 z} H_{2}+\frac{17 z^{2}-28 z+16}{12 z} H_{3} \\
& +\frac{101 z^{2}-193 z+184}{27 z} H_{1}-\frac{52 z^{2}-167 z+134}{36 z} \zeta_{2}+\frac{79 z^{2}-164 z+176}{12 z} \zeta_{3} \\
& -\frac{152 z^{2}-589 z+571}{81 z}+\epsilon\left(-\frac{89 z^{2}-172 z+169}{24 z} \zeta_{2} H_{1,1}-\frac{4 z^{2}+46 z-115}{36 z} H_{1,2}\right.
\end{aligned}
$$




$$
\begin{aligned}
& +\frac{10 z^{2}-8 z-43}{24 z} H_{1,2,1}-\frac{17 z^{2}-28 z+16}{12 z} H_{3,1}-\frac{19 z^{2}-56 z+38}{24 z} H_{2,2} \\
& +\frac{22 z^{2}-14 z-19}{24 z} H_{2,1,1}+\frac{34 z^{2}-74 z+113}{12 z} H_{1,3}-\frac{35 z^{2}-76 z+79}{24 z} H_{1,1,2} \\
& -\frac{44 z^{2}-184 z+193}{24 z} H_{1,1,1}-\frac{68 z^{2}-127 z-20}{72 z} H_{2,1}+\frac{118 z^{2}-236 z+209}{24 z} H_{1,1,1,1} \\
& -\frac{352 z^{2}-548 z+503}{54 z} H_{1,1}+\frac{35 z^{2}-52 z+16}{24 z} \zeta_{2} H_{2}+\frac{104 z^{2}-355 z+304}{36 z} \zeta_{2} H_{1} \\
& -\frac{316 z^{2}-644 z+641}{24 z} \zeta_{3} H_{1}+\frac{17 z^{2}-28 z+16}{3 z} H_{4}+\frac{26 z^{2}-52 z-5}{27 z} H_{2} \\
& +\frac{28 z^{2}-11 z-40}{36 z} H_{3}+\frac{608 z^{2}-2311 z+2194}{162 z} H_{1}+3 \frac{89 z^{2}-236 z+352}{32 z} \zeta_{4} \\
& \left.\left.-\frac{151 z^{2}-464 z+332}{54 z} \zeta_{2}+\frac{472 z^{2}-2057 z+2144}{72 z} \zeta_{3}-\frac{1823 z^{2}-7054 z+6820}{486 z}\right)\right] \\
& +N^{2} N_{f}\left[\frac { 1 } { \epsilon } \left(-\frac{z^{2}-2 z+2}{6 z} H_{1,1}-\frac{z^{2}-2 z+2}{6 z} H_{2}+\frac{z^{2}-2 z+2}{6 z} \zeta_{2}\right.\right. \\
& \left.+\frac{28 z^{2}-47 z+38}{108 z}\right)-\frac{z^{2}-5 z+5}{9 z} H_{1,1}+\frac{z^{2}-2 z+2}{6 z} H_{2,1}-\frac{z^{2}-2 z+2}{3 z} H_{1,2} \\
& +\frac{z^{2}-2 z+2}{2 z} H_{1,1,1}-\frac{z^{2}-2 z+2}{3 z} \zeta_{2} H_{1}-\frac{2\left(z^{2}-2 z+2\right)}{3 z} H_{3} \\
& -\frac{4 z^{2}-17 z+20}{36 z} H_{2}-\frac{56 z^{2}-103 z+94}{108 z} H_{1}-\frac{4\left(z^{2}-2 z+2\right)}{3 z} \zeta_{3} \\
& +\frac{4 z^{2}-17 z+20}{36 z} \zeta_{2}+\frac{80 z^{2}-229 z+130}{324 z}+\epsilon\left(\frac{z^{2}-2 z+2}{3 z} \zeta_{2} H_{1,1}\right. \\
& +\frac{z^{2}-5 z+5}{3 z} H_{1,1,1}-\frac{z^{2}-2 z+2}{6 z} H_{2,1,1}-\frac{z^{2}-2 z+2}{3 z} H_{2,2} \\
& +\frac{z^{2}-2 z+2}{3 z} H_{1,2,1}+\frac{z^{2}-2 z+2}{3 z} H_{1,1,2}+\frac{2\left(z^{2}-2 z+2\right)}{3 z} H_{3,1} \\
& -\frac{7\left(z^{2}-2 z+2\right)}{6 z} H_{1,1,1,1}-\frac{4\left(z^{2}-2 z+2\right)}{3 z} H_{1,3}+\frac{4 z^{2}-17 z+20}{36 z} H_{2,1} \\
& -\frac{8 z^{2}-43 z+40}{36 z} H_{1,2}+\frac{77 z^{2}-49 z+40}{108 z} H_{1,1}-\frac{z^{2}-2 z+2}{3 z} \zeta_{2} H_{2} \\
& +\frac{8\left(z^{2}-2 z+2\right)}{3 z} \zeta_{3} H_{1}-\frac{8 z^{2}-37 z+40}{36 z} \zeta_{2} H_{1}-\frac{8\left(z^{2}-2 z+2\right)}{3 z} H_{4} \\
& -\frac{4 z^{2}-17 z+20}{9 z} H_{3}-\frac{35 z^{2}-151 z+148}{108 z} H_{2}-\frac{160 z^{2}-557 z+458}{324 z} H_{1} \\
& -\frac{3\left(z^{2}-2 z+2\right)}{z} \zeta_{4}+\frac{17 z^{2}-7 z-32}{108 z} \zeta_{2}-\frac{32 z^{2}-175 z+160}{36 z} \zeta_{3} \\
& \left.\left.+\frac{484 z^{2}-1187 z+422}{972 z}\right)\right] \\
& +N\left[\frac { 1 } { \epsilon } \left(\frac{z^{2}-8 z+5}{12 z} H_{1,1}+\frac{z^{2}-11 z+14}{12 z} H_{2}+\frac{1}{4 z} H_{1}+\frac{14 z^{2}-31 z+22}{12 z} \zeta_{2}\right.\right. \\
& \left.+\frac{485 z^{2}-952 z+772}{216 z}\right)+\frac{7 z^{2}-14 z+11}{8 z} H_{1,1,1}+\frac{11 z^{2}-16 z+4}{12 z} H_{2,1} \\
& +\frac{31 z^{2}-80 z+38}{24 z} H_{1,2}+\frac{67 z^{2}-254 z+155}{72 z} H_{1,1}-\frac{53 z^{2}-100 z+88}{24 z} \zeta_{2} H_{1} \\
& +\frac{8 z^{2}-25 z+28}{6 z} H_{3}+\frac{67 z^{2}-350 z+308}{72 z} H_{2}-\frac{485 z^{2}-961 z+736}{108 z} H_{1} \\
& -\frac{85 z^{2}-152 z+176}{12 z} \zeta_{3}+\frac{113 z^{2}-424 z+268}{72 z} \zeta_{2}+\frac{1337 z^{2}-3814 z+2284}{324 z}
\end{aligned}
$$




$$
\begin{aligned}
& +\epsilon\left(\frac{140 z^{2}-358 z+313}{24 z} \zeta_{2} H_{1,1}+\frac{7\left(5 z^{2}-16 z+7\right)}{12 z} H_{1,3}\right. \\
& +\frac{11 z^{2}-100 z+109}{24 z} H_{2,1,1}-\frac{13 z^{2}-20 z+38}{12 z} H_{3,1}-\frac{31 z^{2}-14 z+11}{24 z} H_{1,1,1,1} \\
& +\frac{43 z^{2}-146 z+143}{48 z} H_{1,1,1}+\frac{44 z^{2}-202 z+151}{24 z} H_{1,1,2}+\frac{50 z^{2}-163 z+127}{12 z} H_{2,2} \\
& -\frac{70 z^{2}-104 z+65}{24 z} H_{1,2,1}+\frac{181 z^{2}-146 z+50}{144 z} H_{2,1}+\frac{251 z^{2}-781 z+670}{72 z} H_{1,2} \\
& +\frac{4679 z^{2}-12079 z+9424}{432 z} H_{1,1}+\frac{17 z^{2}-85 z+82}{12 z} \zeta_{2} H_{2} \\
& -\frac{118 z^{2}-503 z+329}{72 z} \zeta_{2} H_{1}+\frac{352 z^{2}-716 z+713}{24 z} \zeta_{3} H_{1}+\frac{2\left(8 z^{2}-25 z+28\right)}{3 z} H_{4} \\
& +\frac{277 z^{2}-1310 z+1232}{72 z} H_{3}+\frac{799 z^{2}-5324 z+3536}{432 z} H_{2}-\frac{1337 z^{2}-3850 z+2194}{162 z} H_{1} \\
& -\frac{188 z^{2}-245 z+528}{16 z} \zeta_{4}-\frac{917 z^{2}-3466 z+4288}{144 z} \zeta_{3}+\frac{2207 z^{2}-7330 z+2656}{432 z} \zeta_{2} \\
& \left.+\frac{3463 z^{2}-8630 z+3410}{243 z}\right) \\
& +N_{f}\left[\frac{1}{\epsilon}\left(\frac{z^{2}-2 z+2}{6 z} H_{1,1}+\frac{z^{2}-2 z+2}{6 z} H_{2}-\frac{z^{2}-2 z+2}{6 z} \zeta_{2}-\frac{28 z^{2}-47 z+38}{108 z}\right)\right. \\
& +\frac{z^{2}-5 z+5}{9 z} H_{1,1}-\frac{z^{2}-2 z+2}{6 z} H_{2,1}+\frac{z^{2}-2 z+2}{3 z} H_{1,2}-\frac{z^{2}-2 z+2}{2 z} H_{1,1,1} \\
& +\frac{z^{2}-2 z+2}{3 z} \zeta_{2} H_{1}+\frac{2\left(z^{2}-2 z+2\right)}{3 z} H_{3}+\frac{4 z^{2}-17 z+20}{36 z} H_{2} \\
& +\frac{56 z^{2}-103 z+94}{108 z} H_{1}+\frac{4\left(z^{2}-2 z+2\right)}{3 z} \zeta_{3}-\frac{4 z^{2}-17 z+20}{36 z} \zeta_{2}-\frac{80 z^{2}-229 z+130}{324 z} \\
& +\epsilon\left(-\frac{z^{2}-2 z+2}{3 z} \zeta_{2} H_{1,1}-\frac{z^{2}-5 z+5}{3 z} H_{1,1,1}+\frac{z^{2}-2 z+2}{6 z} H_{2,1,1}\right. \\
& +\frac{z^{2}-2 z+2}{3 z} H_{2,2}-\frac{z^{2}-2 z+2}{3 z} H_{1,2,1}-\frac{z^{2}-2 z+2}{3 z} H_{1,1,2}-\frac{2\left(z^{2}-2 z+2\right)}{3 z} H_{3,1} \\
& +\frac{7\left(z^{2}-2 z+2\right)}{6 z} H_{1,1,1,1}+\frac{4\left(z^{2}-2 z+2\right)}{3 z} H_{1,3}-\frac{4 z^{2}-17 z+20}{36 z} H_{2,1} \\
& +\frac{8 z^{2}-43 z+40}{36 z} H_{1,2}-\frac{77 z^{2}-49 z+40}{108 z} H_{1,1}+\frac{z^{2}-2 z+2}{3 z} \zeta_{2} H_{2} \\
& -\frac{8\left(z^{2}-2 z+2\right)}{3 z} \zeta_{3} H_{1}+\frac{8 z^{2}-37 z+40}{36 z} \zeta_{2} H_{1}+\frac{8\left(z^{2}-2 z+2\right)}{3 z} H_{4} \\
& +\frac{4 z^{2}-17 z+20}{9 z} H_{3}+\frac{35 z^{2}-151 z+148}{108 z} H_{2}+\frac{160 z^{2}-557 z+458}{324 z} H_{1} \\
& +\frac{3\left(z^{2}-2 z+2\right)}{z} \zeta_{4}-\frac{17 z^{2}-7 z-32}{108 z} \zeta_{2}+\frac{32 z^{2}-175 z+160}{36 z} \zeta_{3} \\
& \left.\left.-\frac{484 z^{2}-1187 z+422}{972 z}\right)\right] \\
& +\frac{1}{N}\left[\frac { 1 } { \epsilon } \left(-\frac{3\left(z^{2}-2 z+2\right)}{8 z} H_{1,1}-\frac{3 z^{2}-10 z+12}{8 z} H_{2}-\frac{z-2}{8 z} H_{1}\right.\right. \\
& \left.+\frac{2-3 z}{8} \zeta_{2}\right)+\frac{3\left(2 z^{2}-4 z+5\right)}{8 z} H_{1,1,1}-\frac{3 z^{2}-5 z+3}{2 z} H_{1,2} \\
& -\frac{4 z^{2}-15 z+18}{8 z} H_{1,1}-\frac{2 z-3}{2 z} H_{2,1}+\frac{3 z^{2}-4 z+3}{4 z} \zeta_{2} H_{1}-\frac{2 z^{2}-9 z+12}{4 z} H_{2} \\
& -\frac{9 z^{2}-22 z+24}{4 z} H_{3}-\frac{z-2}{4 z} H_{1}+\frac{2-3 z}{4} \zeta_{3}+\frac{3-2 z}{4} \zeta_{2}
\end{aligned}
$$




$$
\begin{aligned}
& +\epsilon\left(-\frac{15 z^{2}-26 z+27}{8 z} \zeta_{2} H_{1,1}+\frac{3 z^{2}-2 z+3}{8 z} H_{1,1,2}\right. \\
& -\frac{3\left(4 z^{2}-8 z+11\right)}{8 z} H_{1,1,1,1}-\frac{3\left(7 z^{2}-18 z+14\right)}{8 z} H_{2,2}+\frac{8 z^{2}-34 z+45}{8 z} H_{1,1,1} \\
& -\frac{8 z^{2}-18 z+11}{4 z} H_{1,2}+\frac{9 z^{2}-22 z+24}{4 z} H_{3,1}-\frac{18 z^{2}-32 z+21}{4 z} H_{1,3} \\
& +\frac{18 z^{2}-28 z+15}{8 z} H_{1,2,1}-\frac{3(z-3)(z-2)}{4 z} H_{1,1}-\frac{13 z-24}{8 z} H_{2,1} \\
& +\frac{20 z-21}{8 z} H_{2,1,1}+\frac{4 z^{2}-11 z+10}{4 z} \zeta_{2} H_{1}+\frac{12 z^{2}-16 z+21}{8 z} \zeta_{3} H_{1} \\
& -\frac{9 z^{2}-22 z+24}{z} H_{4}-\frac{12 z^{2}-41 z+48}{4 z} H_{3}-\frac{3(z-4)(z-2)}{8 z} \zeta_{2} H_{2}-\frac{z-2}{2 z} H_{1} \\
& \left.\left.-\frac{3(z-4)(z-2)}{4 z} H_{2}+\frac{15-8 z}{8} \zeta_{3}-\frac{3(z-2)}{4} \zeta_{2}-\frac{87}{32}(3 z-2) \zeta_{4}\right)\right] \\
& +\frac{1}{N^{3}}\left[\frac{1}{\epsilon}\left(\frac{(1-z)(z-3)}{4 z} H_{1,1}+\frac{2-z}{4} H_{2}-\frac{1}{4 z} H_{1}+\frac{z}{2} \zeta_{2}-\frac{3}{8}(z-2)\right)\right. \\
& -\frac{z^{2}-14 z+18}{8 z} H_{1,2}-\frac{7 z^{2}-30 z+19}{8 z} H_{1,1}+\frac{3(1-z)^{2}}{8 z} H_{1,1,1}+\frac{2 z-3}{2 z} H_{2,1} \\
& -\frac{9 z^{2}-14 z+12}{8 z} \zeta_{2} H_{1}+\frac{3 z^{2}-6 z-2}{4 z} H_{1}-\frac{7 z^{2}-26 z+8}{8 z} H_{2}+\frac{2-z}{2} H_{3} \\
& +\frac{3 z+4}{8} \zeta_{2}+\frac{5 z+2}{4} \zeta_{3}-\frac{9}{4}(z-2)+\epsilon\left(-\frac{2 z^{2}-36 z+21}{8 z} \zeta_{2} H_{1,1}\right. \\
& +\frac{z^{2}+6 z-6}{4 z} H_{3,1}+\frac{2 z^{2}-4 z+21}{8 z} H_{1,2,1}-\frac{5 z^{2}-30 z+33}{4 z} H_{1,3} \\
& -\frac{5 z^{2}-14 z+58}{16 z} H_{2,1}-\frac{6 z^{2}-44 z+27}{8 z} H_{1,1,2}-\frac{11 z^{2}-18 z+9}{8 z} H_{2,1,1} \\
& -\frac{57 z^{2}-225 z+128}{16 z} H_{1,1}+\frac{3(1-z)(z-5)}{4 z} H_{2,2}-\frac{(z+3)^{2}}{16 z} H_{1,1,1} \\
& -\frac{(z-2)(11 z-39)}{8 z} H_{1,2}+\frac{(1-z)(17 z-33)}{8 z} H_{1,1,1,1}-\frac{3\left(6 z^{2}-15 z+17\right)}{8 z} \zeta_{2} H_{1} \\
& -\frac{24 z^{2}-40 z+45}{8 z} \zeta_{3} H_{1}+\frac{9 z^{2}-18 z-2}{2 z} H_{1}-\frac{13 z^{2}-66 z+32}{8 z} H_{3} \\
& -\frac{33 z^{2}-156 z+32}{16 z} H_{2}+\frac{(1-z)(5 z-9)}{2 z} \zeta_{2} H_{2}-2(z-2) H_{4}-\frac{5}{16}(5 z-22) \zeta_{2} \\
& \left.\left.+\left(\frac{1}{16}(13 z+42)\right) \zeta_{3}+\left(\frac{1}{16}(185 z+22)\right) \zeta_{4}-\frac{21}{2}(z-2)\right)\right]+\mathcal{O}\left(\epsilon^{2}\right), \\
& V_{g q}^{(2,1)}(z, \epsilon)=3 \epsilon \frac{N^{2}-1}{2 N} \zeta_{3}+\mathcal{O}\left(\epsilon^{2}\right), \\
& V_{g q}^{(2,0)}(z, \epsilon)=N^{3}\left[\frac { 1 } { \epsilon } \left(\frac{31\left(z^{2}-2 z+2\right)}{24 z} H_{1,1}+\frac{31 z^{2}-68 z+80}{24 z} H_{2}+\frac{3 z-4}{8} \zeta_{2}\right.\right. \\
& \left.-\frac{134 z^{2}-241 z+250}{72 z} H_{1}+\frac{z+10}{12}\right)-\frac{10 z^{2}-23 z+29}{6 z} H_{2,1} \\
& -\frac{35 z^{2}-73 z+88}{12 z} H_{1,2}-\frac{40 z^{2}-80 z+89}{24 z} H_{1,1,1}+\frac{104 z^{2}-367 z+358}{72 z} H_{1,1} \\
& -\frac{35 z^{2}-76 z+88}{12 z} H_{3}+\frac{52 z^{2}-227 z+260}{36 z} H_{2}-\frac{88 z^{2}-359 z+368}{54 z} H_{1} \\
& -\frac{z-3}{4 z} \zeta_{2} H_{1}+\frac{4-3 z}{4} \zeta_{3}+\frac{9 z-14}{12} \zeta_{2}-\frac{7}{144}(16 z-119) \\
& +\epsilon\left(\frac{3 z^{2}-4 z-3}{8 z} \zeta_{2} H_{1,1}+\frac{35 z^{2}-88 z+124}{12 z} H_{3,1}+\frac{44 z^{2}-94 z+115}{12 z} H_{1,3}\right.
\end{aligned}
$$




$$
\begin{aligned}
& +\frac{58 z^{2}-146 z+197}{24 z} H_{2,1,1}+\frac{58 z^{2}-116 z+143}{24 z} H_{1,1,1,1} \\
& +\frac{70 z^{2}-152 z+221}{24 z} H_{1,2,1}-\frac{100 z^{2}-422 z+473}{36 z} H_{1,2} \\
& +\frac{115 z^{2}-284 z+374}{24 z} H_{2,2}+\frac{115 z^{2}-236 z+257}{24 z} H_{1,1,2} \\
& -\frac{140 z^{2}-625 z+736}{72 z} H_{2,1}-\frac{140 z^{2}-502 z+493}{72 z} H_{1,1,1} \\
& +\frac{275 z^{2}-1042 z+1006}{108 z} H_{1,1}+\frac{80 z^{2}-156 z+121}{8 z} \zeta_{3} H_{1}+\frac{11 z^{2}-28 z+40}{3 z} H_{4} \\
& -\frac{25 z^{2}-107 z+125}{9 z} H_{3}+\frac{275 z^{2}-1282 z+1492}{108 z} H_{2} \\
& -\frac{527 z^{2}-2149 z+2194}{162 z} H_{1}+\frac{3(z-2)(z+2)}{8 z} \zeta_{2} H_{2}-\frac{5 z-9}{6 z} \zeta_{2} H_{1} \\
& \left.\left.+\frac{17-36 z}{24} \zeta_{3}+\frac{33}{32}(3 z-4) \zeta_{4}+\frac{24 z-53}{18} \zeta_{2}+\frac{22583-4966 z}{864}\right)\right] \\
& +N^{2} N_{f}\left[\frac { 1 } { \epsilon } \left(-\frac{z^{2}-2 z+2}{6 z} H_{1,1}-\frac{z^{2}-2 z+2}{6 z} H_{2}+\frac{5\left(z^{2}-2 z+2\right)}{18 z} H_{1}\right.\right. \\
& \left.-\frac{1+z}{12}\right)-\frac{z^{2}-5 z+5}{9 z} H_{1,1}+\frac{z^{2}-2 z+2}{6 z} H_{2,1}+\frac{z^{2}-2 z+2}{6 z} H_{1,1,1} \\
& +\frac{2\left(z^{2}-2 z+2\right)}{3 z} H_{1,2}-\frac{1}{12} \zeta_{2}+\frac{2\left(z^{2}-2 z+2\right)}{3 z} H_{3}-\frac{4 z^{2}-23 z+20}{36 z} H_{2} \\
& +\frac{26 z^{2}-103 z+94}{108 z} H_{1}-\frac{z+19}{18}+\epsilon\left(\frac{z^{2}-5 z+5}{9 z} H_{1,1,1}\right. \\
& -\frac{z^{2}-2 z+2}{6 z} H_{2,1,1}-\frac{z^{2}-2 z+2}{6 z} H_{1,1,1,1}-\frac{2\left(z^{2}-2 z+2\right)}{3 z} H_{3,1} \\
& -\frac{2\left(z^{2}-2 z+2\right)}{3 z} H_{2,2}-\frac{2\left(z^{2}-2 z+2\right)}{3 z} H_{1,3}-\frac{2\left(z^{2}-2 z+2\right)}{3 z} H_{1,2,1} \\
& -\frac{2\left(z^{2}-2 z+2\right)}{3 z} H_{1,1,2}+\frac{4 z^{2}-23 z+20}{36 z} H_{2,1}+\frac{16 z^{2}-83 z+80}{36 z} H_{1,2} \\
& -\frac{17 z^{2}-49 z+40}{108 z} H_{1,1}-\frac{1}{12} \zeta_{3}+\frac{1}{12} \zeta_{2} H_{1}-\frac{z^{2}-2 z+2}{z} \zeta_{3} H_{1} \\
& +\frac{4\left(z^{2}-5 z+5\right)}{9 z} H_{3}-\frac{2\left(z^{2}-2 z+2\right)}{3 z} H_{4}-\frac{17 z^{2}-55 z+40}{108 z} H_{2} \\
& \left.\left.+\frac{160 z^{2}-557 z+458}{324 z} H_{1}+\left(\frac{1}{36}(13-3 z)\right) \zeta_{2}+\frac{1}{216}(190 z-1331)\right)\right] \\
& +N\left[\frac { 1 } { \epsilon } \left(-\frac{z^{2}-8 z+5}{12 z} H_{1,1}-\frac{z^{2}-11 z+14}{12 z} H_{2}+\frac{134 z^{2}-259 z+259}{36 z} H_{1}\right.\right. \\
& \left.+\frac{3-z}{4} \zeta_{2}+\frac{20-11 z}{24}\right)-\frac{67 z^{2}-272 z+155}{72 z} H_{1,1}+\frac{79 z^{2}-176 z+182}{12 z} H_{2,1} \\
& +\frac{143 z^{2}-316 z+370}{24 z} H_{1,2}+\frac{155 z^{2}-310 z+319}{24 z} H_{1,1,1}-\frac{67 z^{2}-386 z+272}{72 z} H_{2} \\
& +\frac{73 z^{2}-176 z+176}{12 z} H_{3}+\frac{379 z^{2}-1472 z+1382}{108 z} H_{1}-\frac{(z-6)^{2}}{8 z} \zeta_{2} H_{1} \\
& +\frac{52-21 z}{24} \zeta_{2}+\frac{z-3}{2} \zeta_{3}+\frac{1}{144}(635-344 z)+\epsilon\left(\frac{34 z^{2}-42 z+15}{8 z} \zeta_{2} H_{1,1}\right. \\
& +\frac{8 z^{2}-10 z+25}{12 z} H_{1,3}-\frac{28 z^{2}-101 z+65}{12 z} H_{2,2}+\frac{31 z^{2}-14 z+11}{24 z} H_{1,1,1,1} \\
& +\frac{31 z^{2}+16 z-43}{24 z} H_{2,1,1}+\frac{47 z^{2}-64 z+34}{12 z} H_{3,1}-\frac{50 z^{2}-166 z+85}{24 z} H_{1,1,2}
\end{aligned}
$$




$$
\begin{aligned}
& +\frac{100 z^{2}-212 z+203}{24 z} H_{1,2,1}+\frac{535 z^{2}-2321 z+2216}{72 z} H_{1,2} \\
& +\frac{959 z^{2}-3814 z+3859}{144 z} H_{1,1,1}+\frac{1175 z^{2}-5050 z+4858}{144 z} H_{2,1} \\
& -\frac{2203 z^{2}-7775 z+4256}{432 z} H_{1,1}-\frac{36 z^{2}-227 z+327}{24 z} \zeta_{2} H_{1} \\
& -\frac{194 z^{2}-368 z+365}{8 z} \zeta_{3} H_{1}+\frac{z^{2}+25 z-28}{6 z} H_{4}+\frac{643 z^{2}-2468 z+2072}{72 z} H_{3} \\
& +\frac{1135 z^{2}-3929 z+3164}{162 z} H_{1}-\frac{2203 z^{2}-8924 z+4256}{432 z} H_{2} \\
& +\frac{3(2 z-1)(3 z-2)}{4 z} \zeta_{2} H_{2}+\frac{1}{144}(1126-309 z) \zeta_{2}-\frac{5}{16}(19 z-31) \zeta_{4} \\
& \left.\left.+\frac{1}{48}(39 z-346) \zeta_{3}+\frac{1}{864}(16733-9026 z)\right)\right] \\
& +N_{f}\left[\frac{1}{\epsilon}\left(-\frac{z^{2}-2 z+2}{6 z} H_{1,1}-\frac{z^{2}-2 z+2}{6 z} H_{2}-\frac{5\left(z^{2}-2 z+2\right)}{9 z} H_{1}+\frac{z-1}{12}\right)\right. \\
& -\frac{z^{2}-5 z+5}{9 z} H_{1,1}-\frac{5\left(z^{2}-2 z+2\right)}{6 z} H_{2,1}-\frac{5\left(z^{2}-2 z+2\right)}{6 z} H_{1,1,1} \\
& -\frac{4\left(z^{2}-2 z+2\right)}{3 z} H_{1,2}+\frac{1}{12} \zeta_{2}-\frac{4\left(z^{2}-2 z+2\right)}{3 z} H_{3}-\frac{4 z^{2}-17 z+20}{36 z} H_{2} \\
& -\frac{52 z^{2}-215 z+206}{108 z} H_{1}+\frac{1}{36}(8 z+7)+\epsilon\left(-\frac{5\left(z^{2}-5 z+5\right)}{9 z} H_{1,1,1}\right. \\
& -\frac{2\left(z^{2}-2 z+2\right)}{3 z} H_{3,1}-\frac{2\left(z^{2}-2 z+2\right)}{3 z} H_{2,2}-\frac{2\left(z^{2}-2 z+2\right)}{3 z} H_{1,3} \\
& -\frac{2\left(z^{2}-2 z+2\right)}{3 z} H_{1,2,1}-\frac{2\left(z^{2}-2 z+2\right)}{3 z} H_{1,1,2}-\frac{7\left(z^{2}-2 z+2\right)}{6 z} H_{2,1,1} \\
& -\frac{7\left(z^{2}-2 z+2\right)}{6 z} H_{1,1,1,1}-\frac{20 z^{2}-103 z+100}{36 z} H_{2,1}-\frac{32 z^{2}-163 z+160}{36 z} H_{1,2} \\
& -\frac{35 z^{2}-175 z+184}{108 z} H_{1,1}+\frac{1}{12} \zeta_{3}-\frac{1}{12} \zeta_{2} H_{1}+\frac{2\left(z^{2}-2 z+2\right)}{z} \zeta_{3} H_{1} \\
& -\frac{8\left(z^{2}-5 z+5\right)}{9 z} H_{3}-\frac{2\left(z^{2}-2 z+2\right)}{3 z} H_{4}-\frac{35 z^{2}-169 z+184}{108 z} H_{2} \\
& \left.\left.-\frac{320 z^{2}-1213 z+1114}{324 z} H_{1}+\left(\frac{1}{36}(3 z-13)\right) \zeta_{2}+\frac{151-z}{54}\right)\right] \\
& +\frac{1}{N}\left[\frac { 1 } { \epsilon } \left(-\frac{35\left(z^{2}-2 z+2\right)}{24 z} H_{1,1}-\frac{35 z^{2}-58 z+52}{24 z} H_{2}\right.\right. \\
& \left.-\frac{134 z^{2}-295 z+286}{72 z} H_{1}+\frac{1}{8}(3 z-2) \zeta_{2}-\frac{11}{12}\right)-\frac{35 z^{2}-76 z+106}{12 z} H_{1,2} \\
& -\frac{53 z^{2}-118 z+124}{12 z} H_{2,1}-\frac{100 z^{2}-383 z+374}{72 z} H_{1,1} \\
& -\frac{106 z^{2}-212 z+221}{24 z} H_{1,1,1}-\frac{35 z^{2}-82 z+88}{12 z} H_{3}-\frac{50 z^{2}-169 z+142}{36 z} H_{2} \\
& -\frac{230 z^{2}-889 z+862}{108 z} H_{1}-\frac{2 z-15}{4 z} \zeta_{2} H_{1}+\frac{1}{4}(2-3 z) \zeta_{3}+\frac{z-2}{4} \zeta_{2} \\
& +\frac{1}{144}(132 z-793)+\epsilon\left(-\frac{21 z^{2}-46 z+3}{8 z} \zeta_{2} H_{1,1}-\frac{70 z^{2}-128 z+149}{12 z} H_{1,3}\right. \\
& -\frac{77 z^{2}-142 z+199}{24 z} H_{1,1,2}-\frac{77 z^{2}-118 z+190}{24 z} H_{2,2}-\frac{97 z^{2}-158 z+140}{12 z} H_{3,1} \\
& -\frac{100 z^{2}-572 z+725}{36 z} H_{1,2}-\frac{140 z^{2}-280 z+253}{24 z} H_{1,1,1,1}
\end{aligned}
$$




$$
\begin{aligned}
& -\frac{140 z^{2}-220 z+199}{24 z} H_{2,1,1}-\frac{194 z^{2}-364 z+379}{24 z} H_{1,2,1} \\
& -\frac{298 z^{2}-1157 z+1130}{108 z} H_{1,1}-\frac{344 z^{2}-1477 z+1612}{72 z} H_{2,1} \\
& -\frac{344 z^{2}-1396 z+1477}{72 z} H_{1,1,1}-\frac{3\left(7 z^{2}-10 z-4\right)}{8 z} \zeta_{2} H_{2} \\
& +\frac{152 z^{2}-296 z+289}{8 z} \zeta_{3} H_{1}-\frac{35 z^{2}-55 z+52}{6 z} H_{4}-\frac{50 z^{2}-259 z+286}{18 z} H_{3} \\
& -\frac{149 z^{2}-511 z+430}{54 z} H_{2}-\frac{689 z^{2}-2671 z+2590}{162 z} H_{1}-\frac{4 z-9}{z} \zeta_{2} H_{1} \\
& \left.\left.+\frac{1}{8}(23-4 z) \zeta_{3}+\frac{z-2}{2} \zeta_{2}+\left(\frac{3}{32}(73 z-62)\right) \zeta_{4}+\frac{1}{864}(4758 z-20335)\right)\right] \\
& +\frac{N_{f}}{N^{2}}\left[\frac{1}{\epsilon}\left(\frac{z^{2}-2 z+2}{3 z} H_{1,1}+\frac{5\left(z^{2}-2 z+2\right)}{18 z} H_{1}+\frac{z^{2}-2 z+2}{3 z} H_{2}+\frac{1}{6}\right)\right. \\
& +\frac{2\left(z^{2}-5 z+5\right)}{9 z} H_{1,1}+\frac{2\left(z^{2}-2 z+2\right)}{3 z} H_{2,1}+\frac{2\left(z^{2}-2 z+2\right)}{3 z} H_{1,2} \\
& +\frac{2\left(z^{2}-2 z+2\right)}{3 z} H_{1,1,1}+\frac{2\left(z^{2}-5 z+5\right)}{9 z} H_{2}+\frac{2\left(z^{2}-2 z+2\right)}{3 z} H_{3} \\
& +\frac{13 z^{2}-56 z+56}{54 z} H_{1}+\frac{1}{36}(31-6 z)+\epsilon\left(\frac{4\left(z^{2}-5 z+5\right)}{9 z} H_{2,1}\right. \\
& +\frac{4\left(z^{2}-5 z+5\right)}{9 z} H_{1,2}+\frac{4\left(z^{2}-5 z+5\right)}{9 z} H_{1,1,1}+\frac{4\left(z^{2}-2 z+2\right)}{3 z} H_{3,1} \\
& +\frac{4\left(z^{2}-2 z+2\right)}{3 z} H_{2,2}+\frac{4\left(z^{2}-2 z+2\right)}{3 z} H_{2,1,1}+\frac{4\left(z^{2}-2 z+2\right)}{3 z} H_{1,3} \\
& +\frac{4\left(z^{2}-2 z+2\right)}{3 z} H_{1,2,1}+\frac{4\left(z^{2}-2 z+2\right)}{3 z} H_{1,1,2}+\frac{4\left(z^{2}-2 z+2\right)}{3 z} H_{1,1,1,1} \\
& +\frac{13 z^{2}-56 z+56}{27 z} H_{1,1}-\frac{z^{2}-2 z+2}{z} \zeta_{3} H_{1}+\frac{4\left(z^{2}-5 z+5\right)}{9 z} H_{3} \\
& +\frac{4\left(z^{2}-2 z+2\right)}{3 z} H_{4}+\frac{4\left(10 z^{2}-41 z+41\right)}{81 z} H_{1} \\
& \left.\left.+\frac{13 z^{2}-56 z+56}{27 z} H_{2}+\frac{1}{216}(727-186 z)\right)\right] \\
& +\frac{1}{N^{3}}\left[\frac{1}{\epsilon}\left(-\frac{(1-z)(z-3)}{4 z} H_{1,1}+\frac{z-2}{4} H_{2}+\frac{1-z}{4 z} H_{1}-\frac{z}{2} \zeta_{2}+\frac{3(z-2)}{8}\right)\right. \\
& -\frac{z^{2}-6 z-6}{8 z} H_{1,2}+\frac{7 z^{2}-32 z+19}{8 z} H_{1,1}+\frac{2-z}{2} H_{2,1}-\frac{3(1-z)^{2}}{8 z} H_{1,1,1} \\
& +\frac{z^{2}-5 z+8}{4 z} H_{1}+\frac{7 z^{2}-30 z+4}{8 z} H_{2}+\frac{z-6}{8} \zeta_{2} H_{1}+\frac{6-z}{4} H_{3} \\
& -\frac{1}{8}(z+4) \zeta_{2}+z \zeta_{3}+\frac{3}{16}(12 z-25)+\epsilon\left(-\frac{16 z^{2}+9}{8 z} \zeta_{2} H_{1,1}\right. \\
& +\frac{z^{2}+2 z+9}{16 z} H_{1,1,1}+\frac{4 z^{2}-24 z+9}{8 z} H_{1,1,2}+\frac{5 z^{2}-2 z-6}{4 z} H_{3,1} \\
& +\frac{6 z^{2}-8 z+3}{4 z} H_{1,3}+\frac{8 z^{2}-15}{8 z} H_{1,2,1}-\frac{15 z^{2}-37 z-20}{8 z} H_{1,2} \\
& +\frac{17 z^{2}-30 z+15}{8 z} H_{2,1,1}-\frac{23 z^{2}-94 z+18}{16 z} H_{2,1}+\frac{85 z^{2}-305 z+176}{16 z} H_{1,1} \\
& +\frac{3(z-3)(z+1)}{4 z} H_{2,2}-\frac{(1-z)(17 z-33)}{8 z} H_{1,1,1,1}-\frac{3\left(3 z^{2}-2 z+2\right)}{4 z} \zeta_{2} H_{2} \\
& -\frac{38 z^{2}-84 z+45}{8 z} \zeta_{3} H_{1}+\frac{z^{2}-11 z+20}{2 z} H_{1}-\frac{27 z^{2}-64 z-8}{8 z} H_{3}
\end{aligned}
$$




$$
\begin{aligned}
& -\frac{(1-z)(12 z-25)}{8 z} \zeta_{2} H_{1}+(2(z-2)) H_{4}+\frac{(5 z-16)(17 z-4)}{16 z} H_{2} \\
& \left.\left.+\frac{1}{4}(1-16 z) \zeta_{4}+\frac{1}{16}(5 z-62) \zeta_{2}+\frac{1}{16}(19 z+58) \zeta_{3}+\frac{19}{32}(18 z-37)\right)\right]+\mathcal{O}\left(\epsilon^{2}\right), \\
& V_{q q}^{(2,2)}(z, \epsilon)=N^{2}\left[\frac { 1 } { \epsilon } \left(\frac{1-z}{8} H_{1}+\frac{1}{8}(4 z-3) H_{2}+\frac{1}{24}\left(-88 z^{2}+76 z-35\right) \zeta_{2}\right.\right. \\
& \left.-\frac{101}{54}\left(2 z^{2}-2 z+1\right)\right)+\frac{1}{8}\left(-6 z^{2}-2 z+3\right) H_{2,1}+\frac{1}{8}(3 z-2) H_{1,1} \\
& +\frac{1}{4}(1-z)(3 z-2) H_{1,1,1}-\frac{1}{8}(2 z-1)(6 z-5) H_{1,2} \\
& +\frac{1}{24}\left(176 z^{2}-164 z+79\right) \zeta_{2} H_{1}+\frac{1}{2}\left(-3 z^{2}+5 z-3\right) H_{3} \\
& +\frac{1}{216}\left(1616 z^{2}-1697 z+862\right) H_{1}+\frac{1}{8}(11 z-5) H_{2} \\
& +\frac{1}{72}\left(-800 z^{2}+701 z-205\right) \zeta_{2}+\frac{1}{8}\left(62 z^{2}-70 z+37\right) \zeta_{3} \\
& +\frac{1}{162}\left(-1820 z^{2}+1820 z-607\right)+\epsilon\left(\frac{1}{24}\left(-334 z^{2}+310 z-155\right) \zeta_{2} H_{1,1}\right. \\
& +\frac{1}{216}\left(-3232 z^{2}+3475 z-1751\right) H_{1,1}+\frac{1}{8}\left(-18 z^{2}-5 z+5\right) H_{2,1} \\
& +\frac{1}{8}\left(-18 z^{2}+19 z-4\right) H_{1,1,1}+\frac{1}{2}\left(-6 z^{2}+10 z-3\right) H_{1,3} \\
& +\frac{1}{8}\left(-6 z^{2}+14 z-7\right) H_{1,1,2}+\frac{1}{2}\left(3 z^{2}-5 z+3\right) H_{3,1}-\frac{3}{4}\left(5 z^{2}-11 z+5\right) H_{2,2} \\
& +\frac{1}{8}\left(6 z^{2}+26 z-15\right) H_{2,1,1}+\frac{1}{8}\left(24 z^{2}-28 z+9\right) H_{1,2,1} \\
& -\frac{3}{4}(1-z)(3 z-2) H_{1,1,1,1}-\frac{1}{8}(4 z-1)(9 z-8) H_{1,2} \\
& +\frac{1}{8}\left(-124 z^{2}+144 z-71\right) \zeta_{3} H_{1}+\frac{3}{4}\left(z^{2}+z-1\right) \zeta_{2} H_{2} \\
& +\frac{1}{72}\left(1600 z^{2}-1537 z+500\right) \zeta_{2} H_{1}-2\left(3 z^{2}-5 z+3\right) H_{4} \\
& +\frac{7}{648}\left(2080 z^{2}-2161 z+740\right) H_{1}+\frac{1}{2}(1-z)(9 z-5) H_{3}+\frac{1}{8}(25 z-12) H_{2} \\
& +\frac{1}{216}\left(-5632 z^{2}+4957 z-1238\right) \zeta_{2}+\frac{1}{48}\left(-629 z^{2}+107 z+77\right) \zeta_{4} \\
& \left.\left.+\frac{1}{24}\left(562 z^{2}-619 z+239\right) \zeta_{3}-\frac{2}{243}\left(3187 z^{2}-3187 z+911\right)\right)\right] \\
& +N N_{f}\left[\frac{1}{\epsilon}\left(\frac{1}{3}\left(2 z^{2}-2 z+1\right) \zeta_{2}+\frac{7}{27}\left(2 z^{2}-2 z+1\right)\right)\right. \\
& -\frac{2}{3}\left(2 z^{2}-2 z+1\right) \zeta_{2} H_{1}-\frac{14}{27}\left(2 z^{2}-2 z+1\right) H_{1}+\frac{1}{2}\left(-2 z^{2}+2 z-1\right) \zeta_{3} \\
& +\frac{1}{9}\left(16 z^{2}-16 z+5\right) \zeta_{2}+\frac{1}{81}\left(124 z^{2}-124 z+41\right)+\epsilon\left(\frac{4}{3}\left(2 z^{2}-2 z+1\right) \zeta_{2} H_{1,1}\right. \\
& +\frac{28}{27}\left(2 z^{2}-2 z+1\right) H_{1,1}+\left(2 z^{2}-2 z+1\right) \zeta_{3} H_{1}-\frac{2}{9}\left(16 z^{2}-16 z+5\right) \zeta_{2} H_{1} \\
& -\frac{2}{81}\left(124 z^{2}-124 z+41\right) H_{1}+\frac{1}{6}\left(-16 z^{2}+16 z-5\right) \zeta_{3}+\frac{19}{12}\left(2 z^{2}-2 z+1\right) \zeta_{4} \\
& \left.\left.+\frac{4}{27}\left(26 z^{2}-26 z+7\right) \zeta_{2}+\frac{4}{243}\left(215 z^{2}-215 z+61\right)\right)\right] \\
& +\frac{1}{\epsilon}\left(\frac{1}{8}(3-2 z) H_{2}+\frac{1-z}{8} H_{1}+\frac{1}{8}(2 z-3) \zeta_{2}\right)+\frac{1}{8}\left(6 z^{2}-2 z-3\right) H_{2,1}
\end{aligned}
$$




$$
\begin{aligned}
& +\frac{1}{8}\left(12 z^{2}-14 z+1\right) H_{1,2}+\frac{1}{8}(3 z-2) H_{1,1}-\frac{1}{4}(1-z)(3 z-1) H_{1,1,1} \\
& +\frac{1}{2}\left(3 z^{2}-4 z+3\right) H_{3}+\frac{1}{8}(3-2 z) \zeta_{2} H_{1}+\frac{1}{8}(2-3 z) H_{1}+\frac{9(1-z)}{8} H_{2} \\
& +\frac{1}{8}\left(-18 z^{2}+22 z-15\right) \zeta_{3}+\frac{1}{8}(9 z-7) \zeta_{2}+\epsilon\left(\frac{1}{8}\left(-6 z^{2}+10 z-5\right) \zeta_{2} H_{1,1}\right. \\
& +\frac{1}{8}\left(-24 z^{2}+26 z-3\right) H_{1,2,1}+\frac{1}{8}\left(-6 z^{2}-10 z+9\right) H_{2,1,1} \\
& +\frac{1}{2}\left(-3 z^{2}+4 z-3\right) H_{3,1}+\frac{1}{8}\left(6 z^{2}-10 z+5\right) H_{1,1,2} \\
& +\frac{1}{8}\left(22 z^{2}-41 z+16\right) H_{1,1,1}+\frac{1}{8}\left(22 z^{2}-5 z-9\right) H_{2,1} \\
& +\frac{1}{8}\left(44 z^{2}-59 z+18\right) H_{1,2}+z(3 z-4) H_{1,3}+\frac{3}{4}(1-z)(3 z-1) H_{1,1,1,1} \\
& -\frac{3}{4}(1-z)(5 z-3) H_{2,2}+\frac{1}{8}(9 z-5) H_{1,1}+\frac{1}{8}\left(36 z^{2}-46 z+21\right) \zeta_{3} H_{1} \\
& +2\left(3 z^{2}-4 z+3\right) H_{4}+\frac{1}{2}\left(11 z^{2}-16 z+9\right) H_{3}+\frac{1}{8}(10-13 z) \zeta_{2} H_{1} \\
& +\frac{3}{4}\left(1-z^{2}\right) \zeta_{2} H_{2}+\frac{1}{8}(16-23 z) H_{2}+\frac{1}{8}(4-7 z) H_{1}+\frac{1}{8}\left(-66 z^{2}+87 z-35\right) \zeta_{3} \\
& \left.-\frac{3}{16}\left(23 z^{2}-52 z+55\right) \zeta_{4}+\frac{1}{8}(23 z-14) \zeta_{2}\right)+\mathcal{O}\left(\epsilon^{2}\right) \\
& V_{q q}^{(2,1)}(z, \epsilon)=N^{2}\left[\frac{13}{2}\left(2 z^{2}-2 z+1\right) \zeta_{3}+\epsilon\left(-\frac{13}{2}\left(2 z^{2}-2 z+1\right) \zeta_{3} H_{1}\right.\right. \\
& \left.\left.+\frac{39}{4}\left(2 z^{2}-2 z+1\right) \zeta_{4}+\frac{1}{3}\left(119 z^{2}-119 z+40\right) \zeta_{3}\right)\right] \\
& +N N_{f}\left[-2\left(2 z^{2}-2 z+1\right) \zeta_{3}+\epsilon\left(2\left(2 z^{2}-2 z+1\right) \zeta_{3} H_{1}\right.\right. \\
& \left.\left.-3\left(2 z^{2}-2 z+1\right) \zeta_{4}-\frac{2}{3}\left(16 z^{2}-16 z+5\right) \zeta_{3}\right)\right] \\
& +\frac{9}{2}\left(2 z^{2}-2 z+1\right) \zeta_{3}+\epsilon\left(-\frac{9}{2}\left(2 z^{2}-2 z+1\right) \zeta_{3} H_{1}+\frac{27}{4}\left(2 z^{2}-2 z+1\right) \zeta_{4}\right. \\
& \left.+3\left(11 z^{2}-11 z+4\right) \zeta_{3}\right)+\mathcal{O}\left(\epsilon^{2}\right), \\
& V_{q q}^{(2,0)}(z, \epsilon)=N^{2}\left[\frac{37}{24 \epsilon^{3}}\left(2 z^{2}-2 z+1\right)+\frac{1}{\epsilon^{2}}\left(\frac{1}{48}\left(754 z^{2}-754 z+303\right)\right.\right. \\
& \left.-\frac{13}{6}\left(2 z^{2}-2 z+1\right) H_{1}\right)+\frac{1}{\epsilon}\left(\frac{1}{24}\left(-496 z^{2}+499 z-199\right) H_{1}+\frac{1}{8}(3-4 z) H_{2}\right. \\
& \left.+\frac{1}{24}\left(-52 z^{2}+64 z-35\right) \zeta_{2}+\frac{1}{864}\left(50926 z^{2}-50926 z+18677\right)\right) \\
& +\frac{1}{8}\left(-146 z^{2}+154 z-79\right) H_{2,1}+\frac{1}{8}\left(-140 z^{2}+128 z-69\right) H_{1,2} \\
& +\frac{1}{4}\left(-73 z^{2}+71 z-36\right) H_{1,1,1}+\frac{1}{8}(2-3 z) H_{1,1} \\
& +\frac{1}{72}\left(-3856 z^{2}+3883 z-1202\right) H_{1}+\frac{1}{2}\left(-35 z^{2}+37 z-19\right) H_{3} \\
& +\frac{1}{8}(12 z-1) \zeta_{2} H_{1}+\frac{1}{8}(7-15 z) H_{2}+\frac{1}{72}\left(-782 z^{2}+899 z-358\right) \zeta_{2} \\
& +\frac{1}{24}\left(-550 z^{2}+526 z-257\right) \zeta_{3}+\frac{1016318 z^{2}-1016318 z+355381}{5184} \\
& +\epsilon\left(\frac{1}{8}\left(-18 z^{2}+50 z-19\right) \zeta_{2} H_{1,1}+\frac{1}{24}\left(-1330 z^{2}+1327 z-452\right) H_{1,1,1}\right.
\end{aligned}
$$




$$
\begin{aligned}
& +\frac{1}{24}\left(-1330 z^{2}+1405 z-491\right) H_{2,1}+\frac{1}{24}\left(-1276 z^{2}+1201 z-416\right) H_{1,2} \\
& +\frac{1}{8}\left(-36 z^{2}+32 z-13\right) H_{1,2,1}+\frac{1}{8}\left(-18 z^{2}-6 z+7\right) H_{2,1,1} \\
& +\frac{1}{4}\left(-6 z^{2}-2 z+1\right) H_{1,3}+\frac{1}{8}\left(18 z^{2}-34 z+15\right) H_{1,1,2}+\frac{1}{8}(5-9 z) H_{1,1} \\
& +\frac{3}{4}(1-z)(3 z-2) H_{1,1,1,1}+\frac{1}{4}(3 z-7)(3 z-2) H_{2,2}-\frac{3}{2} z(3 z-1) H_{3,1} \\
& +\frac{1}{4}\left(-9 z^{2}+15 z-5\right) \zeta_{2} H_{2}+\frac{1}{8}\left(528 z^{2}-540 z+263\right) \zeta_{3} H_{1} \\
& +\frac{1}{216}\left(-25808 z^{2}+25997 z-7228\right) H_{1}+\frac{1}{12}\left(-638 z^{2}+671 z-235\right) H_{3} \\
& -\frac{3}{2}\left(z^{2}+z-1\right) H_{4}+\frac{1}{8}(35 z-12) \zeta_{2} H_{1}+\frac{1}{8}(18-37 z) H_{2} \\
& +\frac{1}{432}\left(-17402 z^{2}+19076 z-7111\right) \zeta_{2}+\frac{1}{72}\left(-9758 z^{2}+9551 z-3991\right) \zeta_{3} \\
& \left.\left.+\frac{1}{16}\left(77 z^{2}+29 z-41\right) \zeta_{4}+\frac{6380090 z^{2}-6380090 z+2173727}{10368}\right)\right] \\
& +N N_{f}\left[-\frac{5}{12 \epsilon^{3}}\left(2 z^{2}-2 z+1\right)+\frac{1}{\epsilon^{2}}\left(\frac{2}{3}\left(2 z^{2}-2 z+1\right) H_{1}\right.\right. \\
& \left.+\frac{1}{18}\left(-101 z^{2}+101 z-43\right)\right)+\frac{1}{\epsilon}\left(\frac{1}{3}\left(14 z^{2}-14 z+5\right) H_{1}\right. \\
& \left.+\frac{1}{3}\left(2 z^{2}-2 z+1\right) \zeta_{2}+\frac{1}{72}\left(-1622 z^{2}+1622 z-609\right)\right)+2\left(2 z^{2}-2 z+1\right) H_{2,1} \\
& +2\left(2 z^{2}-2 z+1\right) H_{1,2}+2\left(2 z^{2}-2 z+1\right) H_{1,1,1}+2\left(2 z^{2}-2 z+1\right) H_{3} \\
& +\frac{14}{9}\left(7 z^{2}-7 z+2\right) H_{1}+\frac{5}{6}\left(2 z^{2}-2 z+1\right) \zeta_{3}+\frac{1}{18}\left(50 z^{2}-50 z+19\right) \zeta_{2} \\
& +\frac{-97742 z^{2}+97742 z-34273}{1296}+\epsilon\left(\frac{2}{3}\left(16 z^{2}-16 z+5\right) H_{2,1}\right. \\
& +\frac{2}{3}\left(16 z^{2}-16 z+5\right) H_{1,2}+\frac{2}{3}\left(16 z^{2}-16 z+5\right) H_{1,1,1}-6\left(2 z^{2}-2 z+1\right) \zeta_{3} H_{1} \\
& +\frac{2}{3}\left(16 z^{2}-16 z+5\right) H_{3}+\frac{2}{27}\left(311 z^{2}-311 z+82\right) H_{1}-\frac{13}{4}\left(2 z^{2}-2 z+1\right) \zeta_{4} \\
& +\frac{1}{18}\left(464 z^{2}-464 z+217\right) \zeta_{3}+\frac{1}{108}\left(1046 z^{2}-1046 z+373\right) \zeta_{2} \\
& \left.\left.+\frac{-1816750 z^{2}+1816750 z-615149}{7776}\right)\right] \\
& +N_{f}\left[\frac{2}{9 \epsilon^{2}}\left(2 z^{2}-2 z+1\right)+\frac{4}{27 \epsilon}\left(13 z^{2}-13 z+5\right)+\frac{2}{27}\left(80 z^{2}-80 z+27\right)\right. \\
& \left.+\frac{32 \epsilon}{243}\left(121 z^{2}-121 z+38\right)\right] \\
& +\frac{55}{24 \epsilon^{3}}\left(2 z^{2}-2 z+1\right)+\frac{1}{\epsilon^{2}}\left(\frac{1}{72}\left(1504 z^{2}-1504 z+587\right)-\frac{3}{2}\left(2 z^{2}-2 z+1\right) H_{1}\right) \\
& +\frac{1}{\epsilon}\left(\frac{1}{8}\left(-88 z^{2}+89 z-33\right) H_{1}+\frac{1}{8}(2 z-3) H_{2}+\frac{1}{24}\left(-28 z^{2}+22 z-5\right) \zeta_{2}\right. \\
& \left.+\frac{47}{216}\left(334 z^{2}-334 z+119\right)\right)+\frac{1}{8}\left(-36 z^{2}+42 z-11\right) H_{1,2} \\
& +\frac{1}{8}\left(-30 z^{2}+26 z-9\right) H_{2,1}+\frac{1}{4}\left(-15 z^{2}+16 z-7\right) H_{1,1,1}+\frac{1}{8}(2-3 z) H_{1,1} \\
& +\frac{1}{2}\left(-9 z^{2}+8 z-3\right) H_{3}-\frac{3}{8}\left(72 z^{2}-73 z+22\right) H_{1}-\frac{1}{8}(6 z+7) \zeta_{2} H_{1}
\end{aligned}
$$




$$
\begin{aligned}
& +\frac{1}{8}(5 z-7) H_{2}+\frac{1}{24}\left(-538 z^{2}+550 z-287\right) \zeta_{3}+\frac{1}{72}\left(-248 z^{2}+185 z-1\right) \zeta_{2} \\
& +\frac{292994 z^{2}-292994 z+99403}{1296}+\epsilon\left(\frac{1}{8}\left(18 z^{2}-34 z+5\right) \zeta_{2} H_{1,1}\right. \\
& +\frac{1}{8}\left(-132 z^{2}+167 z-54\right) H_{1,2}+\frac{1}{8}\left(-110 z^{2}+95 z-27\right) H_{2,1} \\
& +\frac{1}{8}\left(-110 z^{2}+129 z-48\right) H_{1,1,1}+\frac{1}{8}\left(-18 z^{2}+26 z-9\right) H_{1,1,2} \\
& +\frac{1}{4}\left(-9 z^{2}+18 z-10\right) H_{2,2}+\frac{1}{4}\left(6 z^{2}-2 z+1\right) H_{1,3}+\frac{1}{8}\left(18 z^{2}-6 z-5\right) H_{2,1,1} \\
& +\frac{1}{8}\left(36 z^{2}-34 z+11\right) H_{1,2,1}+\frac{1}{8}(5-9 z) H_{1,1}+\frac{3}{2} z(3 z-2) H_{3,1} \\
& -\frac{3}{4}(1-z)(3 z-1) H_{1,1,1,1}+\frac{1}{4}\left(9 z^{2}-12 z+1\right) \zeta_{2} H_{2} \\
& +\frac{1}{8}\left(-472 z^{2}+479 z-132\right) H_{1}+\frac{1}{4}\left(-66 z^{2}+57 z-17\right) H_{3}-\frac{1}{8}(25 z+2) \zeta_{2} H_{1} \\
& +\frac{1}{8}(6 z+11) \zeta_{3} H_{1}-\frac{3}{2}\left(1-z^{2}\right) H_{4}+\frac{1}{8}(11 z-10) H_{2} \\
& +\frac{1}{72}\left(-10466 z^{2}+10619 z-4615\right) \zeta_{3}+\frac{1}{216}\left(-640 z^{2}+181 z+430\right) \zeta_{2} \\
& \left.+\frac{1}{16}\left(-405 z^{2}+352 z-123\right) \zeta_{4}+\frac{5179370 z^{2}-5179370 z+1710703}{7776}\right) \\
& +\frac{N_{f}}{N}\left[-\frac{5}{12 \epsilon^{3}}\left(2 z^{2}-2 z+1\right)+\frac{1}{18 \epsilon^{2}}\left(-67 z^{2}+67 z-26\right)\right. \\
& +\frac{1}{\epsilon}\left(\frac{1}{6}\left(-2 z^{2}+2 z-1\right) \zeta_{2}+\frac{1}{216}\left(-2786 z^{2}+2786 z-991\right)\right) \\
& +\frac{1}{9}\left(-17 z^{2}+17 z-7\right) \zeta_{2}-\frac{5}{3}\left(2 z^{2}-2 z+1\right) \zeta_{3}-\frac{7\left(6830 z^{2}-6830 z+2221\right)}{1296} \\
& +\epsilon\left(\frac{1}{108}\left(-910 z^{2}+910 z-353\right) \zeta_{2}+\frac{1}{9}\left(-116 z^{2}+116 z-43\right) \zeta_{3}\right. \\
& \left.\left.-3\left(2 z^{2}-2 z+1\right) \zeta_{4}+\frac{-728594 z^{2}+728594 z-220867}{7776}\right)\right] \\
& +\frac{1}{N^{2}}\left[\frac{3}{4 \epsilon^{3}}\left(2 z^{2}-2 z+1\right)+\frac{1}{16 \epsilon^{2}}\left(106 z^{2}-106 z+41\right)\right. \\
& +\frac{1}{\epsilon}\left(\frac{3}{4}\left(2 z^{2}-2 z+1\right) \zeta_{2}+\frac{1}{32}\left(654 z^{2}-654 z+221\right)\right)-\frac{15}{4}\left(2 z^{2}-2 z+1\right) \zeta_{3} \\
& +\frac{1}{8}\left(70 z^{2}-70 z+29\right) \zeta_{2}+\frac{1}{64}\left(3610 z^{2}-3610 z+1151\right) \\
& +\epsilon\left(\frac{1}{2}\left(-107 z^{2}+107 z-46\right) \zeta_{3}-\frac{39}{8}\left(2 z^{2}-2 z+1\right) \zeta_{4}\right. \\
& \left.\left.+\frac{7}{16}\left(82 z^{2}-82 z+31\right) \zeta_{2}+\frac{1}{128}\left(18702 z^{2}-18702 z+5741\right)\right)\right]+\mathcal{O}\left(\epsilon^{2}\right) \text {. }
\end{aligned}
$$

\section{B The CDR matrix elements for $H$ to three partons}

\section{B.1 The matrix element for $H \rightarrow g g g$}

In this section we show how to obtain the two-loop matrix element in CDR from the $D$ dimensional tensor coefficients of ref. [24]. The amplitude for $H \rightarrow g g g$ can be written as

$$
\left|\mathcal{M}_{H \rightarrow g g g}\right\rangle=S_{\mu \nu \rho} \epsilon_{1}^{\mu} \epsilon_{2}^{\nu} \epsilon_{3}^{\rho},
$$


where the gluon tensor is given by,

$$
S_{\mu \nu \rho}=\lambda_{0} \sqrt{4 \pi \alpha_{0}} f^{a_{1} a_{2} a_{3}} \sum_{\ell=0}^{\infty}\left(\frac{\alpha_{0}}{2 \pi}\right)^{\ell}\left(\frac{S_{\epsilon} c_{\Gamma}}{2}\right)^{\ell} e^{i \pi \ell \epsilon}\left(m_{H}^{2}\right)^{-\ell \epsilon} S_{\mu \nu \rho}^{(\ell)},
$$

where $\lambda_{0}$ and $\alpha_{0}$ denote the bare coupling constants, and

$$
S_{\epsilon}=(4 \pi)^{\epsilon} e^{-\gamma_{E} \epsilon} \text { and } c_{\Gamma}=e^{\gamma_{E} \epsilon} \frac{\Gamma(1-\epsilon)^{2} \Gamma(1+\epsilon)}{\Gamma(1-2 \epsilon)},
$$

where $\gamma_{E}=-\Gamma^{\prime}(1)$ denotes the Euler-Mascheroni constant. After tensor decomposition, we get

$$
S_{\mu \nu \rho}^{(\ell)} \epsilon_{1}^{\mu} \epsilon_{2}^{\nu} \epsilon_{3}^{\rho}=\frac{1}{m_{H}^{2}}\left[T_{232} A_{232}^{(\ell)}+T_{211} A_{211}^{(\ell)}+T_{311} A_{311}^{(\ell)}+T_{312} A_{312}^{(\ell)}\right]
$$

where $T_{i j k}$ are the tensors given in eq. (3.7) of ref. [24], and the $A_{i j k}^{(\ell)}$ are scalars. ${ }^{3}$

Our goal is to compute the interference $\left\langle\mathcal{M}_{H \rightarrow g g g}^{(0)} \mid \mathcal{M}_{H \rightarrow g g g}^{(\ell)}\right\rangle$ in $\mathrm{CDR}$, summed over colours and spins. The colour sum is trivial, and gives

$$
f^{a_{1} a_{2} a_{3}} f^{a_{1} a_{2} a_{3}}=V C_{A} .
$$

The polarisation sum read,

$$
\sum_{\text {pol. }} \epsilon_{i \mu}^{*} \epsilon_{i \nu}=-\eta_{\mu \nu}+\text { gauge dependent terms. }
$$

The gauge dependent terms are proportional to the gluon momentum. The tensors $T_{i j k} \equiv$ $T_{i j k}^{\mu \nu \rho} \epsilon_{1}^{\mu} \epsilon_{2}^{\nu} \epsilon_{3}^{\rho}$ of ref. [24] are transverse, i.e., they vanish whenever they are contracted with an external gluon momentum,

$$
T_{i j k}^{\mu \nu \rho} p_{1 \mu}=T_{i j k}^{\mu \nu \rho} p_{2 \nu}=T_{i j k}^{\mu \nu \rho} p_{3 \rho}=0 .
$$

As a consequence, the gauge dependent terms will always drop out, and we can use the 'naive' polarisation sum

$$
\sum_{\text {pol. }} \epsilon_{i \mu}^{*} \epsilon_{i \nu} \rightarrow-\eta_{\mu \nu}
$$

We get

$$
\begin{aligned}
\left\langle\mathcal{M}_{H \rightarrow g g g}^{(0)} \mid \mathcal{M}_{H \rightarrow g g g}^{(\ell)}\right\rangle= & 8 \pi^{2}\left|\lambda_{0}\right|^{2}\left(\frac{\alpha_{0}}{2 \pi}\right)^{\ell+1}\left(\frac{S_{\epsilon} c_{\Gamma}}{2}\right)^{\ell} e^{i \pi \ell \epsilon}\left(m_{H}^{2}\right)^{1-\ell \epsilon} V C_{A} \\
& \times M_{g g g}^{(\ell)}\left(x_{12}, x_{13}, x_{23} ; \epsilon\right)
\end{aligned}
$$

\footnotetext{
${ }^{3}$ We slightly changed the normalisation of the $A_{i j k}^{(\ell)}$ compared to ref. [24], in order to have the colour tensor and the overall coupling constant explicit.
} 
with

$$
\begin{aligned}
M_{g g g}^{(\ell)} & \left(x_{12}, x_{13}, x_{23} ; \epsilon\right) \\
= & A_{211}^{(\ell)} \frac{x_{12}}{2 x_{23}}\left[(D-2)\left(x_{12}^{2}+x_{12} x_{13}+x_{12} x_{23}+x_{13} x_{23}\right)+x_{13}^{2}+x_{23}^{2}\right] \\
& -A_{311}^{(\ell)} \frac{x_{13}}{2 x_{23}}\left[(D-2)\left(x_{13}^{2}+x_{12} x_{13}+x_{12} x_{23}+x_{13} x_{23}\right)+x_{12}^{2}+x_{23}^{2}\right] \\
& +A_{232}^{(\ell)} \frac{x_{23}}{2 x_{13}}\left[(D-2)\left(x_{23}^{2}+x_{12} x_{13}+x_{12} x_{23}+x_{13} x_{23}\right)+x_{12}^{2}+x_{13}^{2}\right] \\
& -\frac{1}{2} A_{312}^{(\ell)}\left[(D-2)\left(x_{12}^{2}+x_{23}^{2}+x_{13}^{2}\right)+(3 D-8)\left(x_{12} x_{13}+x_{12} x_{23}+x_{13} x_{23}\right)\right],
\end{aligned}
$$

where $x_{i j}=s_{i j} / m_{H}^{2}$ and $x_{12}+x_{13}+x_{23}=1$, and where we have used the fact that

$$
\begin{array}{ll}
A_{211}^{(0)}=\frac{2}{x_{13}}, & A_{311}^{(0)}=-\frac{2}{x_{12}}, \\
A_{232}^{(0)}=\frac{2}{x_{12}}, & A_{312}^{(0)}=-\frac{2}{x_{12}}-\frac{2}{x_{13}}-\frac{2}{x_{23}} .
\end{array}
$$

\section{B.2 The matrix element for $H \rightarrow q \bar{q} g$}

The amplitude for $H \rightarrow q \bar{q} g$ can be written as

$$
\begin{aligned}
\left|\mathcal{M}_{H \rightarrow q \bar{q} g}\right\rangle & =\lambda_{0} \sqrt{4 \pi \alpha_{0}} T_{i j}^{a} \sum_{\ell=0}^{\infty}\left(\frac{\alpha_{0}}{2 \pi}\right)^{\ell}\left(\frac{S_{\epsilon} c_{\Gamma}}{2}\right)^{\ell} e^{i \pi \ell \epsilon}\left(m_{H}^{2}\right)^{-\ell \epsilon} T_{\rho}^{(\ell)} \epsilon_{3}^{\rho} \\
& =\lambda_{0} \sqrt{4 \pi \alpha_{0}} T_{i j}^{a}\left[T_{1} A_{1}+T_{2} A_{2}\right]
\end{aligned}
$$

where as in the gluon case we have factored out explicitly the overall coupling and colour structure. We have

$$
T_{i}=\bar{u}_{1} \gamma_{\mu} v_{2}\left[p_{3}^{\mu} \epsilon_{3} \cdot p_{i}-\epsilon_{3}^{\mu} p_{3} \cdot p_{i}\right] .
$$

The colour sum is trivial and the quake spin sum give

$$
\sum_{\text {spins }}\left[\bar{u}_{1} \gamma_{\mu} v_{2}\right]^{\dagger} \bar{u}_{1} \gamma_{\nu} v_{2}=\operatorname{Tr}\left[\not p / \gamma_{\nu} \not p_{2} \gamma_{\mu}\right]=4\left(p_{1 \mu} p_{2 \nu}+p_{1 \nu} p_{2 \mu}-\frac{1}{2} s_{12} \eta_{\mu \nu}\right)
$$

The tensors $T_{i} \equiv T_{i \rho} \epsilon_{3}^{\rho}$ are transverse, $T_{i \rho} p_{3}^{\rho}=0$, and so we can again use the 'naive' polarisation sum for the gluons. We get,

$$
\begin{aligned}
\left\langle\mathcal{M}_{H \rightarrow q \bar{q} g}^{(0)} \mid \mathcal{M}_{H \rightarrow q \bar{q} g}^{(\ell)}\right\rangle= & 8 \pi^{2}\left|\lambda_{0}\right|^{2}\left(\frac{\alpha_{0}}{2 \pi}\right)^{\ell+1}\left(\frac{S_{\epsilon} c_{\Gamma}}{2}\right)^{\ell} C_{F} C_{A} e^{i \pi \ell \epsilon}\left(m_{H}^{2}\right)^{1-\ell \epsilon} \\
& \times M_{q \bar{q} g}^{(\ell)}\left(x_{12}, x_{13}, x_{23} ; \epsilon\right)
\end{aligned}
$$

where we used the fact that $A_{i}^{(0)}=1 / x_{12}$ and where we defined

$$
\begin{aligned}
M_{q \bar{q} g}^{(\ell)}\left(x_{12}, x_{13}, x_{23} ; \epsilon\right)= & \frac{1}{2} A_{1}^{(\ell)} x_{13}\left[(D-2) x_{13}+(D-4) x_{23}\right] \\
& +\frac{1}{2} A_{2}^{(\ell)} x_{23}\left[(D-4) x_{13}+(D-2) x_{23}\right]
\end{aligned}
$$




\section{Two-loop single-real contributions to Higgs production at $\mathrm{N}^{3} \mathrm{LO}$}

In this appendix we present the result for the contributions of the two-loop amplitude for $H+3$ partons to the inclusive gluon-fusion cross section at $\mathrm{N}^{3} \mathrm{LO}$ computed in section 4 . The results are expressed in terms of harmonic polylogarithms up to weight five, and we use the shorthand $H_{i, \ldots, j} \equiv H_{i, \ldots, j}(z)$, where $z=m_{H}^{2} / s$. The results for the three different initial states are given in the subsequent sections. In all cases we have factored out the leading-order inclusive cross section,

$$
\sigma_{0}=\frac{\pi \lambda_{0}^{2}}{8 V v^{2}}
$$

where $v \simeq 246 \mathrm{GeV}$ is the vacuum expectation value of the Higgs field.

\section{C.1 The $g g$ initial state}

The contribution of the two-loop amplitude for $g g \rightarrow H g$ to the inclusive Higgs cross section at $\mathrm{N}^{3} \mathrm{LO}$ can be written as

$$
\hat{\sigma}_{g g \rightarrow H g}^{(3)}=2\left(\frac{\alpha_{0}}{2 \pi}\right)^{3} s^{-1-3 \epsilon} \sigma_{0}\left(\hat{\sigma}_{g g \rightarrow H g}^{(3), s i n g}+\hat{\sigma}_{g g \rightarrow H g}^{(3), r e g}\right) .
$$

The first term represents the soft-virtual term, which is entirely determined by the QCD soft current,

$$
\hat{\sigma}_{g g \rightarrow H g}^{(3), s i n g}=\sum_{k=0}^{2} \bar{z}^{-1-2(k+1) \epsilon} \frac{\Gamma(-(k+1) \epsilon)^{2}}{\Gamma(-2(k+1) \epsilon)} \mathbf{S}_{g}^{(2, k)}(\epsilon),
$$

where $\mathbf{S}_{g}^{(2, k)}(\epsilon)$ is defined in eq. (4.19). The second term is regular as $z \rightarrow 1$, and can be written as

$$
\hat{\sigma}_{g g \rightarrow H g}^{(3), r e g}=\sum_{k=-5}^{0} \epsilon^{k}\left[N^{3} A_{g g}^{(k)}+N^{2} N_{f} B_{g g}^{(k)}+N N_{f}^{2} C_{g g}^{(k)}+N_{f} D_{g g}^{(k)}\right],
$$

with

$$
\begin{aligned}
A_{g g}^{(-5)}= & \frac{19}{6} z\left(z^{2}-z+2\right), \\
B_{g g}^{(-5)}= & 0, \\
C_{g g}^{(-5)}= & 0, \\
D_{g g}^{(-5)}= & 0, \\
A_{g g}^{(-4)}= & \frac{7\left(z^{2}-z+1\right)^{2}}{3(1-z)} H_{0}+9 z\left(z^{2}-z+2\right) H_{1} \\
& +\frac{1}{72}\left(371 z^{3}-965 z^{2}+1039 z-297\right), \\
B_{g g}^{(-4)}= & \frac{7}{18} z\left(z^{2}-z+2\right), \\
C_{g g}^{(-4)}= & 0, \\
D_{g g}^{(-4)}= & 0,
\end{aligned}
$$




$$
\begin{aligned}
A_{g g}^{(-3)}= & \frac{8\left(z^{2}-z+1\right)^{2}}{3(1-z)} H_{1,0}-\frac{19\left(z^{2}-z+1\right)^{2}}{3(1-z)} H_{0,0}+30 z\left(z^{2}-z+2\right) H_{1,1} \\
& -\frac{\left(z^{2}-z+1\right)^{2}}{9(1-z)} H_{0}+\frac{8\left(z^{2}-z+1\right)^{2}}{3(1-z)} H_{2}+\frac{1}{4}\left(69 z^{3}-179 z^{2}+193 z-55\right) H_{1} \\
& -\frac{143}{4} z\left(z^{2}-z+2\right) \zeta_{2}+\frac{1}{432}\left(5291 z^{3}-16889 z^{2}+15361 z-6723\right) \\
B_{g g}^{(-3)}= & \frac{4\left(z^{2}-z+1\right)^{2}}{9(1-z)} H_{0}+z\left(z^{2}-z+2\right) H_{1} \\
& +\frac{1}{216}\left(323 z^{3}-473 z^{2}+745 z-99\right) \\
C_{g g}^{(-3)}= & 0 \\
D_{g g}^{(-3)}= & 0
\end{aligned}
$$

$$
\begin{aligned}
A_{g g}^{(-2)}= & -\frac{8\left(z^{2}-z+1\right)^{2}}{3(1-z)} H_{2,1}-\frac{8\left(z^{2}-z+1\right)^{2}}{3(1-z)} H_{1,2}-\frac{16\left(z^{2}-z+1\right)^{2}}{3(1-z)} H_{1,1,0} \\
& -\frac{40\left(z^{2}-z+1\right)^{2}}{3(1-z)} H_{2,0}-\frac{40\left(z^{2}-z+1\right)^{2}}{3(1-z)} H_{1,0,0}+\frac{17\left(z^{2}-z+1\right)^{2}}{1-z} H_{0,0,0} \\
& +116 z\left(z^{2}-z+2\right) H_{1,1,1}+\frac{1}{6}\left(389 z^{3}-1027 z^{2}+1097 z-319\right) H_{1,1} \\
& -\frac{10 z^{4}-30 z^{3}+30 z^{2}-10 z-1}{1-z} H_{0,0}-\frac{95 z^{4}-388 z^{3}+582 z^{2}-388 z+95}{18(1-z)} H_{1,0} \\
& -\frac{287\left(z^{2}-z+1\right)^{2}}{6(1-z)} \zeta_{2} H_{0}-\frac{22\left(z^{2}-z+1\right)^{2}}{3(1-z)} H_{3} \\
& +\frac{1}{72}\left(2915 z^{3}-8969 z^{2}+8361 z-3467\right) H_{1}-\frac{97 z^{4}-284 z^{3}+291 z^{2}-104 z-2}{9(1-z)} H_{2} \\
& +\frac{545 z^{4}-1090 z^{3}+1635 z^{2}-1090 z-16}{6(1-z)} \zeta_{2} H_{1} \\
& -\frac{274 z^{4}-653 z^{3}+1035 z^{2}-656 z+274}{54(1-z)} H_{0} \\
& +\frac{57601 z^{3}-149875 z^{2}+147119 z-58485}{1296} \\
& +\frac{1}{144}\left(-6853 z^{3}+19051 z^{2}-17645 z+5379\right) \zeta_{2} \\
& +\frac{121 z^{4}-242 z^{3}+363 z^{2}-242 z-10}{3(1-z)} \zeta_{3}, \\
C_{g g}^{(-2)}= & \frac{z^{2}}{27}, \quad \frac{239}{36} z\left(z^{2}-z+2\right) \zeta_{2}, \\
& +\frac{4\left(z^{2}-z+1\right)^{2}}{9(1-z)} H_{1,0}-\frac{4\left(z^{2}-z+1\right)^{2}}{3(1-z)} H_{0,0}+\frac{10}{3} z\left(z^{2}-z+2\right) H_{1,1} \\
& +\frac{64 z^{4}-125 z^{3}+189 z^{2}-128 z+64}{54(1-z)} H_{0}+\frac{1}{648}\left(1435 z^{3}-4207 z^{2}+4541 z-1509\right) \\
B_{g g}^{(-2)}= & \left.151 z^{3}-233 z^{2}+357 z-55\right) H_{1} \\
& \\
& \\
&
\end{aligned}
$$




$$
\begin{aligned}
& D_{g g}^{(-2)}=\frac{1}{2} z\left(z^{2}-z+2\right) \\
& A_{g g}^{(-1)}=\frac{324799 z^{3}-729868 z^{2}+781709 z-239112}{1944}+\frac{14\left(z^{2}-z+1\right)^{2}}{3(1-z)} H_{3,1} \\
& -\frac{34\left(z^{2}-z+1\right)^{2}}{3(1-z)} H_{1,3}+\frac{18\left(z^{2}-z+1\right)^{2}}{1-z} H_{2,0,0}+\frac{20\left(z^{2}-z+1\right)^{2}}{1-z} H_{4} \\
& -\frac{32\left(z^{2}-z+1\right)^{2}}{1-z} H_{1,1,0,0}-\frac{98\left(z^{2}-z+1\right)^{2}}{3(1-z)} H_{2,1,0}-\frac{98\left(z^{2}-z+1\right)^{2}}{3(1-z)} H_{1,2,0} \\
& -\frac{100\left(z^{2}-z+1\right)^{2}}{3(1-z)} H_{2,2}+\frac{34\left(z^{2}-z+1\right)^{2}}{1-z} H_{3,0}+\frac{40\left(z^{2}-z+1\right)^{2}}{1-z} H_{1,0,0,0} \\
& -\frac{124\left(z^{2}-z+1\right)^{2}}{3(1-z)} H_{2,1,1}-\frac{124\left(z^{2}-z+1\right)^{2}}{3(1-z)} H_{1,2,1}-\frac{45\left(z^{2}-z+1\right)^{2}}{1-z} H_{0,0,0,0} \\
& -\frac{152\left(z^{2}-z+1\right)^{2}}{3(1-z)} H_{1,1,2}-\frac{208\left(z^{2}-z+1\right)^{2}}{3(1-z)} H_{1,1,1,0}+504 z\left(z^{2}-z+2\right) H_{1,1,1,1} \\
& +\frac{1}{864}\left(-101659 z^{3}+339529 z^{2}-262625 z+133107\right) \zeta_{2} \\
& -\frac{z\left(77 z^{3}-190 z^{2}+195 z-60\right)}{18(1-z)} H_{3}+\frac{1}{3}\left(799 z^{3}-2185 z^{2}+2291 z-693\right) H_{1,1,1} \\
& +\frac{1}{36}\left(5559 z^{3}-16881 z^{2}+15857 z-6479\right) H_{1,1} \\
& +\frac{1}{216}\left(27345 z^{3}-74861 z^{2}+71343 z-26931\right) H_{1} \\
& -\frac{152 z^{4}-610 z^{3}+915 z^{2}-600 z+141}{18(1-z)} H_{1,0,0} \\
& +\frac{179 z^{4}-570 z^{3}+560 z^{2}-180 z-30}{6(1-z)} H_{0,0,0} \\
& -\frac{323 z^{4}-1372 z^{3}+2058 z^{2}-1372 z+323}{9(1-z)} H_{1,1,0} \\
& -\frac{407 z^{4}-1143 z^{3}+956 z^{2}-220 z-286}{18(1-z)} H_{0,0} \\
& -\frac{499 z^{4}-2030 z^{3}+3045 z^{2}-2020 z+488}{18(1-z)} H_{1,2} \\
& -\frac{526 z^{4}-1520 z^{3}+1545 z^{2}-540 z-24}{18(1-z)} H_{2,0} \\
& +\frac{541 z^{4}+1513 z^{3}-2448 z^{2}-614 z+1630}{162(1-z)} H_{0} \\
& -\frac{607 z^{4}-1784 z^{3}+1821 z^{2}-644 z-20}{9(1-z)} H_{2,1} \\
& -\frac{1597 z^{4}-5681 z^{3}+8427 z^{2}-5684 z+1597}{54(1-z)} H_{1,0} \\
& -\frac{2335 z^{4}-6035 z^{3}+6978 z^{2}-3278 z+256}{54(1-z)} H_{2} \\
& +\frac{3443 z^{4}-12394 z^{3}+17931 z^{2}-11554 z+2498}{36(1-z)} \zeta_{3}
\end{aligned}
$$




$$
\begin{aligned}
& -\frac{16783 z^{4}-33566 z^{3}+50349 z^{2}-33566 z-2976}{96(1-z)} \zeta_{4}-\frac{11\left(z^{2}-z+1\right)^{2}}{1-z} \zeta_{3} H_{0} \\
& -\frac{184\left(z^{2}-z+1\right)^{2}}{3(1-z)} \zeta_{2} H_{2}-\frac{250\left(z^{2}-z+1\right)^{2}}{3(1-z)} \zeta_{2} H_{1,0}+\frac{265\left(z^{2}-z+1\right)^{2}}{2(1-z)} \zeta_{2} H_{0,0} \\
& +\frac{7\left(121 z^{4}-242 z^{3}+363 z^{2}-242 z-8\right)}{3(1-z)} \zeta_{2} H_{1,1} \\
& +\frac{2\left(233 z^{4}-466 z^{3}+699 z^{2}-466 z-19\right)}{3(1-z)} \zeta_{3} H_{1} \\
& -\frac{331 z^{4}-1010 z^{3}+960 z^{2}-270 z-87}{6(1-z)} \zeta_{2} H_{0} \\
& +\frac{9059 z^{4}-36400 z^{3}+54600 z^{2}-36400 z+9389}{72(1-z)} \zeta_{2} H_{1}, \\
& B_{g g}^{(-1)}=-\frac{8\left(z^{2}-z+1\right)^{2}}{9(1-z)} H_{2,1}-\frac{16\left(z^{2}-z+1\right)^{2}}{9(1-z)} H_{1,1,0}+\frac{44}{3} z\left(z^{2}-z+2\right) H_{1,1,1} \\
& +\frac{1}{18}\left(281 z^{3}-471 z^{2}+683 z-121\right) H_{1,1}-\frac{2 z^{4}-133 z^{3}+195 z^{2}-130 z+2}{54(1-z)} H_{1,0} \\
& -\frac{14 z^{4}-34 z^{3}+51 z^{2}-35 z+16}{18(1-z)} H_{1,2}+\frac{26 z^{4}-49 z^{3}+73 z^{2}-48 z+24}{6(1-z)} H_{0,0,0} \\
& -\frac{44 z^{4}-91 z^{3}+138 z^{2}-93 z+48}{18(1-z)} H_{2,0}-\frac{46 z^{4}-98 z^{3}+147 z^{2}-99 z+48}{18(1-z)} H_{1,0,0} \\
& -\frac{108 z^{4}-251 z^{3}+319 z^{2}-176 z+64}{18(1-z)} H_{0,0}+\frac{1}{36}\left(299 z^{3}-772 z^{2}+889 z-260\right) H_{1} \\
& -\frac{64 z^{4}-131 z^{3}+198 z^{2}-133 z+68}{6(1-z)} \zeta_{2} H_{0} \\
& +\frac{301 z^{4}-602 z^{3}+903 z^{2}-602 z-20}{18(1-z)} \zeta_{2} H_{1}-\frac{22 z^{4}-50 z^{3}+75 z^{2}-51 z+24}{18(1-z)} H_{3} \\
& -\frac{68 z^{4}-259 z^{3}+213 z^{2}-22 z-64}{54(1-z)} H_{2} \\
& -\frac{2\left(94 z^{4}-221 z^{3}+342 z^{2}-224 z+103\right)}{81(1-z)} H_{0} \\
& +\frac{1}{432}\left(-11707 z^{3}+18121 z^{2}-25601 z+3003\right) \zeta_{2} \\
& +\frac{-16744 z^{3}-8897 z^{2}-20960 z-11313}{1944}+\frac{161 z^{4}-304 z^{3}+453 z^{2}-298 z-16}{18(1-z)} \zeta_{3}, \\
& C_{g g}^{(-1)}=\frac{4 z^{2}}{27} H_{1}+\frac{1}{162}\left(-9 z^{3}+44 z^{2}-3 z+12\right) \\
& D_{g g}^{(-1)}=\frac{\left(z^{2}-z+1\right)^{2}}{1-z} H_{0}+z\left(z^{2}-z+2\right) H_{1}-4 z\left(z^{2}-z+2\right) \zeta_{3} \\
& +\frac{1}{24}\left(157 z^{3}-181 z^{2}+325 z-11\right), \\
& A_{g g}^{(0)}=\frac{6917536 z^{3}-14883745 z^{2}+16560434 z-3796749}{11664} \\
& -\frac{4\left(z^{2}-z+1\right)^{2}}{1-z} H_{4,1}-\frac{32\left(z^{2}-z+1\right)^{2}}{3(1-z)} H_{1,1,3}+\frac{94\left(z^{2}-z+1\right)^{2}}{3(1-z)} H_{2,2,0}
\end{aligned}
$$




$$
\begin{aligned}
& +\frac{32\left(z^{2}-z+1\right)^{2}}{1-z} H_{2,1,0,0}+\frac{104\left(z^{2}-z+1\right)^{2}}{3(1-z)} H_{2,3}-\frac{36\left(z^{2}-z+1\right)^{2}}{1-z} H_{2,0,0,0} \\
& +\frac{110\left(z^{2}-z+1\right)^{2}}{3(1-z)} H_{1,3,1}+\frac{40\left(z^{2}-z+1\right)^{2}}{1-z} H_{1,4}-\frac{48\left(z^{2}-z+1\right)^{2}}{1-z} H_{3,0,0} \\
& -\frac{54\left(z^{2}-z+1\right)^{2}}{1-z} H_{5}+\frac{80\left(z^{2}-z+1\right)^{2}}{1-z} H_{3,1,0}+\frac{82\left(z^{2}-z+1\right)^{2}}{1-z} H_{3,2} \\
& -\frac{84\left(z^{2}-z+1\right)^{2}}{1-z} H_{4,0}+\frac{88\left(z^{2}-z+1\right)^{2}}{1-z} H_{1,3,0}+\frac{278\left(z^{2}-z+1\right)^{2}}{3(1-z)} H_{3,1,1} \\
& -\frac{286\left(z^{2}-z+1\right)^{2}}{3(1-z)} H_{2,1,2}-\frac{96\left(z^{2}-z+1\right)^{2}}{1-z} H_{1,2,2}-\frac{292\left(z^{2}-z+1\right)^{2}}{3(1-z)} H_{2,2,1} \\
& -\frac{296\left(z^{2}-z+1\right)^{2}}{3(1-z)} H_{1,1,1,0,0}-\frac{102\left(z^{2}-z+1\right)^{2}}{1-z} H_{1,1,2,0}-\frac{308\left(z^{2}-z+1\right)^{2}}{3(1-z)} H_{2,1,1,0} \\
& -\frac{308\left(z^{2}-z+1\right)^{2}}{3(1-z)} H_{1,2,1,0}-\frac{108\left(z^{2}-z+1\right)^{2}}{1-z} H_{1,0,0,0,0}+\frac{114\left(z^{2}-z+1\right)^{2}}{1-z} H_{1,1,0,0,0} \\
& +\frac{117\left(z^{2}-z+1\right)^{2}}{1-z} H_{0,0,0,0,0}-\frac{776\left(z^{2}-z+1\right)^{2}}{3(1-z)} H_{2,1,1,1}-\frac{776\left(z^{2}-z+1\right)^{2}}{3(1-z)} H_{1,2,1,1} \\
& -\frac{880\left(z^{2}-z+1\right)^{2}}{3(1-z)} H_{1,1,2,1}-\frac{360\left(z^{2}-z+1\right)^{2}}{1-z} H_{1,1,1,2}-\frac{1408\left(z^{2}-z+1\right)^{2}}{3(1-z)} H_{1,1,1,1,0} \\
& +2384 z\left(z^{2}-z+2\right) H_{1,1,1,1,1}+\frac{2}{3}\left(1757 z^{3}-5035 z^{2}+5153 z-1639\right) H_{1,1,1,1} \\
& +\frac{1}{9}\left(5835 z^{3}-17949 z^{2}+16690 z-6991\right) H_{1,1,1}+\frac{94\left(z^{2}-z+1\right)^{2}}{3(1-z)} H_{1,2,0,0} \\
& -\frac{67 z^{4}-236 z^{3}+224 z^{2}-66 z-21}{1-z} H_{0,0,0,0}-\frac{2\left(73 z^{4}-56 z^{3}+84 z^{2}-46 z+62\right)}{9(1-z)} H_{1,3} \\
& +\frac{1}{216}\left(96 z^{4}+88650 z^{3}-222173 z^{2}+221368 z-61997\right) H_{1} \\
& +\frac{115 z^{4}-512 z^{3}+213 z^{2}+228 z-336}{18(1-z)} H_{2,0,0} \\
& +\frac{170 z^{4}-1834 z^{3}+2751 z^{2}-1824 z+192}{18(1-z)} H_{1,0,0,0} \\
& -\frac{287 z^{4}-831 z^{3}+889 z^{2}-356 z+34}{3(1-z)} H_{2,2} \\
& +\frac{515 z^{4}-1648 z^{3}+1617 z^{2}-528 z-90}{9(1-z)} H_{3,1} \\
& +\frac{569 z^{4}-1654 z^{3}+1671 z^{2}-564 z-36}{18(1-z)} H_{4} \\
& -\frac{2\left(637 z^{4}-2504 z^{3}+3756 z^{2}-2494 z+626\right)}{9(1-z)} H_{1,2,1} \\
& -\frac{730 z^{4}-2462 z^{3}+3693 z^{2}-2412 z+675}{18(1-z)} H_{1,1,0,0} \\
& -\frac{797 z^{4}-2674 z^{3}+4011 z^{2}-2684 z+775}{18(1-z)} H_{1,2,0} \\
& +\frac{889 z^{4}-2886 z^{3}+1911 z^{2}-114 z-1788}{36(1-z)} H_{0,0,0}
\end{aligned}
$$




$$
\begin{aligned}
& +\frac{1415 z^{4}-4246 z^{3}+4209 z^{2}-1356 z-180}{18(1-z)} H_{3,0} \\
& +\frac{1586 z^{4}-3172 z^{3}+4758 z^{2}-3172 z-5225}{15(1-z)} \zeta_{5} \\
& -\frac{2051 z^{4}-5686 z^{3}+5919 z^{2}-2196 z+60}{18(1-z)} H_{2,1,0} \\
& -\frac{2939 z^{4}-16984 z^{3}+27480 z^{2}-21564 z+5169}{108(1-z)} H_{1,0,0} \\
& -\frac{3079 z^{4}-12596 z^{3}+18894 z^{2}-12556 z+3035}{18(1-z)} H_{1,1,2} \\
& -\frac{3199 z^{4}-9368 z^{3}+9597 z^{2}-3428 z-68}{9(1-z)} H_{2,1,1} \\
& -\frac{3461 z^{4}-9748 z^{3}+8103 z^{2}-1416 z-1608}{108(1-z)} H_{3} \\
& -\frac{3657 z^{4}-16002 z^{3}+24003 z^{2}-15992 z+3646}{18(1-z)} H_{1,1,1,0} \\
& -\frac{4006 z^{4}-16085 z^{3}+23490 z^{2}-16073 z+4006}{27(1-z)} H_{1,1,0} \\
& -\frac{5006 z^{4}-13312 z^{3}+12237 z^{2}-3831 z-1680}{54(1-z)} H_{2,0} \\
& -\frac{12580 z^{4}-32927 z^{3}+35517 z^{2}-15170 z-656}{54(1-z)} H_{2,1} \\
& -\frac{14311 z^{4}-56954 z^{3}+86754 z^{2}-61564 z+16541}{108(1-z)} H_{1,2} \\
& +\frac{117637 z^{4}-412340 z^{3}+574362 z^{2}-369668 z+90169}{432(1-z)} \zeta_{3} \\
& +\frac{148347 z^{4}-240328 z^{3}+138972 z^{2}+57432 z-92695}{384(1-z)} \zeta_{4} \\
& +\frac{1}{108}\left(-48 z^{5}-495 z^{4}+48370 z^{3}-137141 z^{2}+126868 z-47810\right) H_{1,1} \\
& -\frac{216 z^{5}-54056 z^{4}+88189 z^{3}-113004 z^{2}+84694 z-51428}{486(1-z)} H_{0} \\
& +\frac{48 z^{6}+447 z^{5}-11754 z^{4}+25186 z^{3}-28256 z^{2}+17101 z-2540}{108(1-z)} H_{0,0} \\
& +\frac{144 z^{6}+1341 z^{5}-32230 z^{4}+88754 z^{3}-104124 z^{2}+47015 z+4340}{324(1-z)} H_{2} \\
& +\frac{144 z^{6}+1341 z^{5}-23266 z^{4}+95600 z^{3}-136668 z^{2}+86531 z-16354}{324(1-z)} H_{1,0} \\
& -\frac{3456 z^{6}+32184 z^{5}-1450121 z^{4}+4685548 z^{3}-6395130 z^{2}+4498564 z-1391205}{2592(1-z)} \zeta_{2} \\
& +\frac{70\left(z^{2}-z+1\right)^{2}}{3(1-z)} \zeta_{3} H_{1,0}+\frac{89\left(z^{2}-z+1\right)^{2}}{3(1-z)} \zeta_{3} H_{0,0}-\frac{116\left(z^{2}-z+1\right)^{2}}{3(1-z)} \zeta_{2} H_{2,1} \\
& -\frac{116\left(z^{2}-z+1\right)^{2}}{3(1-z)} \zeta_{2} H_{1,2}+\frac{154\left(z^{2}-z+1\right)^{2}}{3(1-z)} \zeta_{3} H_{2}-\frac{310\left(z^{2}-z+1\right)^{2}}{3(1-z)} \zeta_{2} H_{1,1,0}
\end{aligned}
$$




$$
\begin{aligned}
& +\frac{7463\left(z^{2}-z+1\right)^{2}}{48(1-z)} \zeta_{4} H_{0}+\frac{168\left(z^{2}-z+1\right)^{2}}{1-z} \zeta_{2} H_{2,0}+\frac{169\left(z^{2}-z+1\right)^{2}}{1-z} \zeta_{2} H_{3} \\
& +\frac{318\left(z^{2}-z+1\right)^{2}}{1-z} \zeta_{2} H_{1,0,0}-\frac{729\left(z^{2}-z+1\right)^{2}}{2(1-z)} \zeta_{2} H_{0,0,0} \\
& +\frac{4\left(171 z^{4}-342 z^{3}+513 z^{2}-342 z-13\right)}{1-z} \zeta_{3} H_{1,1} \\
& +\frac{583 z^{4}-1772 z^{3}+1098 z^{2}+388 z-770}{18(1-z)} \zeta_{3} H_{0} \\
& +\frac{667 z^{4}-2018 z^{3}+2847 z^{2}-1848 z+810}{18(1-z)} \zeta_{2} H_{2} \\
& +\frac{1037 z^{4}-1024 z^{3}+1536 z^{2}-1164 z+1389}{36(1-z)} \zeta_{2} H_{1,0} \\
& +\frac{1433 z^{4}-4492 z^{3}+4328 z^{2}-1302 z-327}{6(1-z)} \zeta_{2} H_{0,0} \\
& -\frac{1549 z^{4}-3098 z^{3}+4647 z^{2}-3098 z+141}{3(1-z)} \zeta_{2} \zeta_{3} \\
& +\frac{2\left(1657 z^{4}-3314 z^{3}+4971 z^{2}-3314 z-164\right)}{3(1-z)} \zeta_{2} H_{1,1,1} \\
& -\frac{3016 z^{4}-8611 z^{3}+4033 z^{2}+1762 z-5078}{36(1-z)} \zeta_{2} H_{0} \\
& +\frac{6008 z^{4}-23614 z^{3}+35421 z^{2}-23634 z+5745}{18(1-z)} \zeta_{3} H_{1} \\
& -\frac{11359 z^{4}-22718 z^{3}+34077 z^{2}-22718 z-7040}{48(1-z)} \zeta_{4} H_{1} \\
& +\frac{17457 z^{4}-70956 z^{3}+106434 z^{2}-71096 z+19249}{36(1-z)} \zeta_{2} H_{1,1} \\
& +\frac{128729 z^{4}-615292 z^{3}+899310 z^{2}-632356 z+226457}{432(1-z)} \zeta_{2} H_{1} \\
& B_{g g}^{(0)}=\frac{-9697813 z^{3}+6173047 z^{2}-15914939 z-2575755}{116640} \\
& -\frac{80\left(z^{2}-z+1\right)^{2}}{9(1-z)} H_{2,1,1}+\frac{232}{3} z\left(z^{2}-z+2\right) H_{1,1,1,1} \\
& +\frac{1}{9}\left(671 z^{3}-1206 z^{2}+1661 z-319\right) H_{1,1,1} \\
& +\frac{-3168 z^{4}-11889 z^{3}-411311 z^{2}+373833 z-287025}{6480} H_{1} \\
& +\frac{2\left(10 z^{4}-14 z^{3}+21 z^{2}-13 z+8\right)}{9(1-z)} H_{1,3}-\frac{14 z^{4}-25 z^{3}+37 z^{2}-24 z+12}{1-z} H_{0,0,0,0} \\
& +\frac{2\left(14 z^{4}-22 z^{3}+33 z^{2}-21 z+12\right)}{9(1-z)} H_{3,1}-\frac{28 z^{4}-49 z^{3}+73 z^{2}-48 z+24}{6(1-z)} H_{2,2} \\
& -\frac{2\left(32 z^{4}-70 z^{3}+105 z^{2}-71 z+34\right)}{9(1-z)} H_{1,2,1}-\frac{58 z^{4}-110 z^{3}+165 z^{2}-109 z+62}{18(1-z)} H_{1,2,0} \\
& -\frac{68 z^{4}-154 z^{3}+231 z^{2}-159 z+78}{18(1-z)} H_{1,1,0,0}-\frac{70 z^{4}-164 z^{3}+255 z^{2}-177 z+96}{18(1-z)} H_{2,1,0}
\end{aligned}
$$




$$
\begin{aligned}
& +\frac{70 z^{4}-146 z^{3}+219 z^{2}-147 z+72}{18(1-z)} H_{4}-\frac{97 z^{4}-200 z^{3}+300 z^{2}-202 z+101}{9(1-z)} H_{1,1,2} \\
& +\frac{110 z^{4}-235 z^{3}+354 z^{2}-237 z+120}{18(1-z)} H_{2,0,0}+\frac{121 z^{4}-431 z^{3}+222 z^{2}+120 z-192}{54(1-z)} H_{3} \\
& +\frac{142 z^{4}-290 z^{3}+435 z^{2}-291 z+144}{18(1-z)} H_{3,0} \\
& +\frac{238 z^{4}-482 z^{3}+723 z^{2}-483 z+234}{18(1-z)} H_{1,0,0,0} \\
& -\frac{269 z^{4}-13 z^{3}-1113 z^{2}+1896 z-687}{54(1-z)} H_{1,0,0} \\
& -\frac{277 z^{4}-566 z^{3}-294 z^{2}+1358 z-679}{54(1-z)} H_{1,2} \\
& -\frac{354 z^{4}-714 z^{3}+1071 z^{2}-715 z+356}{18(1-z)} H_{1,1,1,0} \\
& -\frac{392 z^{4}-1285 z^{3}+1926 z^{2}-1297 z+392}{27(1-z)} H_{1,1,0} \\
& -\frac{854 z^{4}-2389 z^{3}+2643 z^{2}-1108 z+128}{54(1-z)} H_{2,1} \\
& -\frac{968 z^{4}-2287 z^{3}+3147 z^{2}-1860 z+768}{108(1-z)} H_{2,0} \\
& +\frac{1010 z^{4}-2427 z^{3}+2829 z^{2}-1380 z+384}{36(1-z)} H_{0,0,0} \\
& -\frac{6405 z^{4}-12286 z^{3}+18219 z^{2}-11946 z-400}{96(1-z)} \zeta_{4} \\
& +\frac{9409 z^{4}-26048 z^{3}+43110 z^{2}-33572 z+6973}{216(1-z)} \zeta_{3} \\
& +\frac{1}{540}\left(264 z^{5}+900 z^{4}+23560 z^{3}-60085 z^{2}+68650 z-18689\right) H_{1,1} \\
& +\frac{9504 z^{5}-867377 z^{4}+2107411 z^{3}-3141027 z^{2}+1899229 z-749000}{19440(1-z)} H_{0} \\
& -\frac{528 z^{6}+1272 z^{5}+11805 z^{4}-30200 z^{3}+3475 z^{2}+15820 z-8240}{1080(1-z)} H_{0,0} \\
& -\frac{1584 z^{6}+3816 z^{5}+48155 z^{4}-184075 z^{3}+275505 z^{2}-177889 z+54824}{3240(1-z)} H_{1,0} \\
& -\frac{1584 z^{6}+3816 z^{5}+63740 z^{4}-159985 z^{3}+122820 z^{2}-26755 z+8240}{3240(1-z)} H_{2} \\
& +\frac{9504 z^{6}+22896 z^{5}+363505 z^{4}-1459070 z^{3}+1999530 z^{2}-1225646 z+272361}{6480(1-z)} \zeta_{2} \\
& -\frac{47 z^{4}-52 z^{3}+72 z^{2}-40 z+20}{9(1-z)} \zeta_{3} H_{0}+\frac{202 z^{4}-395 z^{3}+595 z^{2}-396 z+204}{6(1-z)} \zeta_{2} H_{0,0} \\
& -\frac{268 z^{4}-635 z^{3}+975 z^{2}-672 z+348}{18(1-z)} \zeta_{2} H_{2}+\frac{308 z^{4}-616 z^{3}+924 z^{2}-615 z-15}{9(1-z)} \zeta_{3} H_{1} \\
& -\frac{430 z^{4}-902 z^{3}+1353 z^{2}-909 z+462}{18(1-z)} \zeta_{2} H_{1,0} \\
& -\frac{465 z^{4}-1128 z^{3}+1421 z^{2}-766 z+272}{9(1-z)} \zeta_{2} H_{0}
\end{aligned}
$$




$$
\begin{aligned}
& +\frac{954 z^{4}-1854 z^{3}+2781 z^{2}-1847 z-194}{18(1-z)} \zeta_{2} H_{1,1} \\
& +\frac{5\left(2857 z^{4}-7088 z^{3}+9834 z^{2}-5756 z+25\right)}{216(1-z)} \zeta_{2} H_{1} \\
& C_{g g}^{(0)}=\frac{16 z^{2}}{27} H_{1,1}+\frac{1}{81}\left(-9 z^{3}+79 z^{2}+3 z+15\right) H_{1}-\frac{z^{2}}{6} \zeta_{2} \\
& +\frac{1}{162}\left(-81 z^{3}+230 z^{2}-67 z+112\right) \\
& D_{g g}^{(0)}=\frac{1}{45}\left(2 z^{5}-30 z^{4}+165 z^{3}-140 z^{2}+210 z-27\right) H_{1,1} \\
& -\frac{2 z^{6}-32 z^{5}+15 z^{4}+55 z^{3}-190 z^{2}+123 z-63}{45(1-z)} H_{1,0} \\
& -\frac{4 z^{6}-64 z^{5}+390 z^{4}-525 z^{3}+720 z^{2}-540 z+270}{90(1-z)} H_{0,0} \\
& -\frac{1}{3} z(4 z-3) H_{1,0,0}-\frac{1}{3} z(4 z-3) H_{1,2}-\frac{8\left(z^{2}-z+1\right)^{2}}{1-z} \zeta_{3} H_{0}-8 z\left(z^{2}-z+2\right) \zeta_{3} H_{1} \\
& +\frac{1}{180}\left(-8 z^{4}+2201 z^{3}-2796 z^{2}+5028 z-255\right) H_{1} \\
& +\frac{8 z^{5}+2501 z^{4}-4843 z^{3}+6921 z^{2}-4632 z+2370}{180(1-z)} H_{0} \\
& -\frac{4 z^{6}-64 z^{5}+30 z^{4}+195 z^{3}-360 z^{2}+180 z-90}{90(1-z)} H_{2}+\frac{1}{3} z(4 z-3) \zeta_{2} H_{1} \\
& -\frac{20}{3} z\left(z^{2}-z+2\right) \zeta_{4}+\frac{1}{3}\left(-69 z^{3}+86 z^{2}-146 z+11\right) \zeta_{3} \\
& +\frac{1}{720}\left(29441 z^{3}-39619 z^{2}+64213 z-5225\right) \\
& +\frac{24 z^{6}-384 z^{5}+2685 z^{4}-4210 z^{3}+5305 z^{2}-3666 z+396}{180(1-z)} \zeta_{2} .
\end{aligned}
$$

\section{C.2 The $g q$ initial state}

The contribution of the two-loop amplitude for $g q \rightarrow H q$ to the inclusive Higgs cross section at $\mathrm{N}^{3} \mathrm{LO}$ can be written as

$$
\hat{\sigma}_{g q \rightarrow H q}^{(3)}=2\left(\frac{\alpha_{0}}{2 \pi}\right)^{3} s^{-1-3 \epsilon} \sigma_{0} \hat{\sigma}_{g q \rightarrow H q}^{(3)} .
$$

Note that, unlike for the pure-gluon initial state, the result is regular in the limit $z \rightarrow 1$, and so we do not need to separate off the contribution from the soft limit. The coefficient $\hat{\sigma}_{g q \rightarrow H q}^{(3)}$ can be written as

$$
\begin{gathered}
\hat{\sigma}_{g q \rightarrow H q}^{(3)}=\sum_{k=-5}^{0} \epsilon^{k}[ \\
N^{3} A_{g q}^{(k)}+N^{2} N_{f} B_{g q}^{(k)}+N N_{f}^{2} C_{g q}^{(k)}+N_{f} D_{g q}^{(k)}+N E_{g q}^{(k)} \\
\left.+\frac{N_{f}^{2}}{N} F_{g q}^{(k)}+\frac{1}{N} G_{g q}^{(k)}+\frac{N_{f}}{N^{2}} H_{g q}^{(k)}+\frac{1}{N^{3}} I_{g q}^{(k)}\right]
\end{gathered}
$$


with

$$
\begin{aligned}
& A_{g q}^{(-5)}=-\frac{1}{4}\left(z^{2}-2 z+2\right), \\
& B_{g q}^{(-5)}=0 \text {, } \\
& C_{g q}^{(-5)}=0 \text {, } \\
& D_{g q}^{(-5)}=0 \text {, } \\
& E_{g q}^{(-5)}=\frac{3}{8}\left(z^{2}-2 z+2\right), \\
& F_{g q}^{(-5)}=0 \text {, } \\
& G_{g q}^{(-5)}=-\frac{7}{48}\left(z^{2}-2 z+2\right), \\
& H_{g q}^{(-5)}=0 \text {, } \\
& I_{g q}^{(-5)}=\frac{1}{48}\left(z^{2}-2 z+2\right), \\
& A_{g q}^{(-4)}=\frac{1}{4}\left(z^{2}-2 z+2\right) H_{0}-\frac{5}{8}\left(z^{2}-2 z+2\right) H_{1}+\frac{1}{8}(z+1)^{2}, \\
& B_{g q}^{(-4)}=-\frac{1}{8}\left(z^{2}-2 z+2\right) \text {, } \\
& C_{g q}^{(-4)}=0 \text {, } \\
& D_{g q}^{(-4)}=\frac{23}{144}\left(z^{2}-2 z+2\right), \\
& E_{g q}^{(-4)}=-\frac{7}{24}\left(z^{2}-2 z+2\right) H_{0}+\frac{25}{24}\left(z^{2}-2 z+2\right) H_{1} \\
& +\frac{1}{288}\left(89 z^{2}-394 z+178\right) \text {, } \\
& F_{g q}^{(-4)}=0 \text {, } \\
& G_{g q}^{(-4)}=-\frac{1}{2}\left(z^{2}-2 z+2\right) H_{1}+\frac{1}{24}\left(z^{2}-2 z+2\right) H_{0} \\
& +\frac{1}{288}\left(-170 z^{2}+424 z-289\right) \text {, } \\
& H_{g q}^{(-4)}=-\frac{5}{144}\left(z^{2}-2 z+2\right), \\
& I_{g q}^{(-4)}=\frac{1}{12}\left(z^{2}-2 z+2\right) H_{1}+\frac{1}{96}\left(15 z^{2}-34 z+25\right), \\
& A_{g q}^{(-3)}=\frac{7}{24}\left(z^{2}-2 z+2\right) H_{1,0}-\frac{2}{3}\left(z^{2}-2 z+2\right) H_{0,0}-\frac{23}{12}\left(z^{2}-2 z+2\right) H_{1,1} \\
& +\frac{1}{48}\left(-19 z^{2}+14 z-14\right) H_{0}+\frac{7}{24}\left(z^{2}-2 z+2\right) H_{2}+\frac{1}{16}\left(5 z^{2}+10 z+8\right) H_{1} \\
& +\frac{27}{8}\left(z^{2}-2 z+2\right) \zeta_{2}+\frac{1471 z^{2}-2942 z+2780}{1728}, \\
& B_{g q}^{(-3)}=\frac{1}{12}\left(z^{2}-2 z+2\right) H_{0}-\frac{3}{8}\left(z^{2}-2 z+2\right) H_{1} \\
& +\frac{1}{432}\left(-164 z^{2}+436 z-301\right) \text {, } \\
& C_{g q}^{(-3)}=-\frac{1}{54}\left(z^{2}-2 z+2\right), \\
& D_{g q}^{(-3)}=-\frac{1}{12}\left(z^{2}-2 z+2\right) H_{0}+\frac{37}{72}\left(z^{2}-2 z+2\right) H_{1} \\
& +\frac{1}{288}\left(189 z^{2}-470 z+335\right) \text {, }
\end{aligned}
$$




$$
\begin{aligned}
& E_{g q}^{(-3)}=-\frac{1}{3}\left(z^{2}-2 z+2\right) H_{1,0}+\frac{19}{24}\left(z^{2}-2 z+2\right) H_{0,0}+\frac{41}{12}\left(z^{2}-2 z+2\right) H_{1,1} \\
& -\frac{1}{3}\left(z^{2}-2 z+2\right) H_{2}+\frac{1}{12}\left(4 z^{2}-z+1\right) H_{0}+\frac{1}{144}\left(160 z^{2}-620 z+269\right) H_{1} \\
& -\frac{35}{8}\left(z^{2}-2 z+2\right) \zeta_{2}+\frac{802 z^{2}-3104 z+971}{1728}, \\
& F_{g q}^{(-3)}=\frac{1}{54}\left(z^{2}-2 z+2\right) \text {, } \\
& G_{g q}^{(-3)}=-\frac{1}{8}\left(z^{2}-2 z+2\right) H_{0,0}+\frac{1}{24}\left(z^{2}-2 z+2\right) H_{1,0}-\frac{11}{6}\left(z^{2}-2 z+2\right) H_{1,1} \\
& +\frac{1}{144}\left(-295 z^{2}+734 z-491\right) H_{1}+\frac{1}{24}\left(z^{2}-2 z+2\right) H_{2} \\
& +\frac{1}{48}\left(3 z^{2}-10 z+10\right) H_{0}+\frac{35}{32}\left(z^{2}-2 z+2\right) \zeta_{2}+\frac{-3830 z^{2}+9664 z-6181}{1728}, \\
& H_{g q}^{(-3)}=\frac{1}{864}\left(-239 z^{2}+538 z-403\right)-\frac{5}{36}\left(z^{2}-2 z+2\right) H_{1}, \\
& I_{g q}^{(-3)}=\frac{1}{3}\left(z^{2}-2 z+2\right) H_{1,1}+\frac{1}{24}\left(15 z^{2}-34 z+25\right) H_{1}-\frac{3}{32}\left(z^{2}-2 z+2\right) \zeta_{2} \\
& +\frac{1}{192}\left(173 z^{2}-402 z+270\right) \text {, } \\
& A_{g q}^{(-2)}=-\frac{1}{48}\left(55 z^{2}-82 z+46\right) H_{1,0}-\frac{1}{8}\left(z^{2}-2 z+2\right) H_{2,1} \\
& +\frac{1}{8}\left(z^{2}-2 z+2\right) H_{1,2}-\frac{3}{8}\left(z^{2}-2 z+2\right) H_{1,1,0}-\frac{25}{24}\left(z^{2}-2 z+2\right) H_{1,0,0} \\
& -\frac{31}{24}\left(z^{2}-2 z+2\right) H_{2,0}+\frac{7}{4}\left(z^{2}-2 z+2\right) H_{0,0,0}-\frac{29}{4}\left(z^{2}-2 z+2\right) H_{1,1,1} \\
& +\frac{1}{48}\left(3 z^{2}+10 z+50\right) H_{0,0}+\frac{1}{144}\left(89 z^{2}+374 z+247\right) H_{1,1} \\
& -\frac{127}{24}\left(z^{2}-2 z+2\right) \zeta_{2} H_{0}+\frac{347}{48}\left(z^{2}-2 z+2\right) \zeta_{2} H_{1}+\frac{1}{48}\left(-73 z^{2}+70 z-10\right) H_{2} \\
& +\frac{1}{72}\left(-67 z^{2}+146 z-137\right) H_{0}-\frac{19}{24}\left(z^{2}-2 z+2\right) H_{3} \\
& +\frac{1}{864}\left(2357 z^{2}-4930 z+4201\right) H_{1}+\frac{1}{16}\left(-16 z^{2}-60 z-29\right) \zeta_{2} \\
& +\frac{23}{8}\left(z^{2}-2 z+2\right) \zeta_{3}+\frac{18356 z^{2}-52852 z+29659}{10368} \\
& B_{g q}^{(-2)}=-\frac{1}{4}\left(z^{2}-2 z+2\right) H_{0,0}+\frac{1}{12}\left(z^{2}-2 z+2\right) H_{1,0}-\frac{47}{36}\left(z^{2}-2 z+2\right) H_{1,1} \\
& +\frac{1}{432}\left(-485 z^{2}+1294 z-835\right) H_{1}+\frac{1}{12}\left(z^{2}-2 z+2\right) H_{2} \\
& +\frac{1}{36}\left(5 z^{2}-16 z+16\right) H_{0}+\frac{23}{16}\left(z^{2}-2 z+2\right) \zeta_{2}+\frac{-1135 z^{2}+4346 z-1862}{2592}, \\
& C_{g q}^{(-2)}=\frac{1}{324}\left(-47 z^{2}+106 z-79\right)-\frac{2}{27}\left(z^{2}-2 z+2\right) H_{1}, \\
& D_{g q}^{(-2)}=-\frac{1}{12}\left(z^{2}-2 z+2\right) H_{1,0}+\frac{1}{4}\left(z^{2}-2 z+2\right) H_{0,0}+\frac{67}{36}\left(z^{2}-2 z+2\right) H_{1,1} \\
& +\frac{1}{36}\left(-5 z^{2}+16 z-16\right) H_{0}-\frac{1}{12}\left(z^{2}-2 z+2\right) H_{2} \\
& +\frac{1}{144}\left(321 z^{2}-790 z+547\right) H_{1}-\frac{455}{288}\left(z^{2}-2 z+2\right) \zeta_{2}
\end{aligned}
$$




$$
\begin{aligned}
& +\frac{5123 z^{2}-13666 z+8023}{2592} \\
& E_{g q}^{(-2)}=\frac{5}{24}\left(z^{2}-2 z+2\right) H_{1,2}+\frac{1}{3}\left(z^{2}-2 z+2\right) H_{2,1}+\frac{2}{3}\left(z^{2}-2 z+2\right) H_{1,1,0} \\
& +\frac{37}{24}\left(z^{2}-2 z+2\right) H_{1,0,0}+\frac{5}{3}\left(z^{2}-2 z+2\right) H_{2,0}-\frac{17}{8}\left(z^{2}-2 z+2\right) H_{0,0,0} \\
& +\frac{79}{6}\left(z^{2}-2 z+2\right) H_{1,1,1}+\frac{1}{24}\left(35 z^{2}-54 z+27\right) H_{1,0} \\
& +\frac{1}{48}\left(51 z^{2}-82 z-20\right) H_{0,0}+\frac{1}{72}\left(334 z^{2}-1160 z+485\right) H_{1,1} \\
& +\frac{287}{48}\left(z^{2}-2 z+2\right) \zeta_{2} H_{0}-\frac{521}{48}\left(z^{2}-2 z+2\right) \zeta_{2} H_{1}+\frac{11}{12}\left(z^{2}-2 z+2\right) H_{3} \\
& +\frac{1}{144}\left(101 z^{2}-205 z+196\right) H_{0}+\frac{1}{432}\left(316 z^{2}-1763 z+467\right) H_{1} \\
& +\frac{1}{48} z(115 z-102) H_{2}+\frac{1}{576}\left(-2159 z^{2}+8206 z-2824\right) \zeta_{2}-\frac{75}{16}\left(z^{2}-2 z+2\right) \zeta_{3} \\
& +\frac{1778 z^{2}-7064 z+4481}{3456} \\
& F_{g q}^{(-2)}=\frac{2}{27}\left(z^{2}-2 z+2\right) H_{1}+\frac{1}{324}\left(47 z^{2}-106 z+79\right) \\
& G_{g q}^{(-2)}=\frac{1}{144}\left(-1117 z^{2}+2762 z-1817\right) H_{1,1}+\frac{1}{16}\left(-21 z^{2}+26 z-10\right) H_{0,0} \\
& -\frac{5}{24}\left(z^{2}-2 z+2\right) H_{2,1}-\frac{7}{24}\left(z^{2}-2 z+2\right) H_{1,1,0}+\frac{3}{8}\left(z^{2}-2 z+2\right) H_{0,0,0} \\
& -\frac{3}{8}\left(z^{2}-2 z+2\right) H_{2,0}-\frac{11}{24}\left(z^{2}-2 z+2\right) H_{1,2}-\frac{5}{8}\left(z^{2}-2 z+2\right) H_{1,0,0} \\
& -\frac{29}{4}\left(z^{2}-2 z+2\right) H_{1,1,1}-\frac{1}{48}(3 z-4)(5 z-2) H_{1,0}-\frac{11}{16}\left(z^{2}-2 z+2\right) \zeta_{2} H_{0} \\
& +\frac{33}{8}\left(z^{2}-2 z+2\right) \zeta_{2} H_{1}+\frac{1}{432}\left(-2957 z^{2}+7684 z-4930\right) H_{1} \\
& +\frac{1}{48}\left(-51 z^{2}+38 z+10\right) H_{2}+\frac{1}{8}\left(-z^{2}+2 z-2\right) H_{3}+\frac{1}{24}\left(7 z^{2}-16 z+13\right) H_{0} \\
& +\frac{13}{6}\left(z^{2}-2 z+2\right) \zeta_{3}+\frac{1}{576}\left(3212 z^{2}-6964 z+4471\right) \zeta_{2} \\
& +\frac{-67970 z^{2}+179128 z-109873}{10368} \\
& H_{g q}^{(-2)}=-\frac{5}{9}\left(z^{2}-2 z+2\right) H_{1,1}+\frac{1}{216}\left(-239 z^{2}+538 z-403\right) H_{1} \\
& +\frac{41}{288}\left(z^{2}-2 z+2\right) \zeta_{2}+\frac{-3988 z^{2}+9320 z-6161}{2592}, \\
& I_{g q}^{(-2)}=\frac{1}{8}\left(z^{2}-2 z+2\right) H_{1,0,0}+\frac{1}{8}\left(z^{2}-2 z+2\right) H_{1,2}+\frac{4}{3}\left(z^{2}-2 z+2\right) H_{1,1,1} \\
& +\frac{1}{6}\left(15 z^{2}-34 z+25\right) H_{1,1}+\frac{1}{16} z(3 z-2) H_{0,0}-\frac{1}{2}\left(z^{2}-2 z+2\right) \zeta_{2} H_{1} \\
& +\frac{1}{96}\left(325 z^{2}-768 z+525\right) H_{1}+\frac{1}{16}(1-z) z H_{0}+\frac{1}{16} z(3 z-2) H_{2} \\
& +\frac{1}{64}\left(-53 z^{2}+102 z-67\right) \zeta_{2}-\frac{17}{48}\left(z^{2}-2 z+2\right) \zeta_{3}+\frac{1}{384}\left(1640 z^{2}-3892 z+2473\right), \\
& A_{g q}^{(-1)}=\frac{-41678 z^{2}-16840 z-79505}{20736}+\frac{1}{384}\left(-3871 z^{2}+6334 z-8048\right) \zeta_{2}
\end{aligned}
$$




$$
\begin{aligned}
& +\frac{1}{144}\left(-206 z^{2}+751 z-262\right) H_{2}+\frac{1}{48}\left(-163 z^{2}+312 z-136\right) H_{1,2} \\
& +\frac{1}{48}\left(-163 z^{2}+362 z-110\right) H_{1,1,0}+\frac{1}{72}\left(-127 z^{2}+353 z-284\right) H_{1,0} \\
& +\frac{1}{16}\left(-91 z^{2}+90 z+30\right) H_{2,1}+\frac{1}{144}\left(-56 z^{2}-560 z-421\right) \zeta_{3} \\
& +\frac{1}{432}\left(-43 z^{2}+161 z+154\right) H_{0}+\frac{1}{48}\left(-11 z^{2}+24 z+130\right) H_{2,0} \\
& +\frac{1}{24}\left(-z^{2}-6 z-87\right) H_{0,0,0}+\frac{1}{24}\left(-z^{2}+2 z-2\right) H_{3,1}-\frac{13}{12}\left(z^{2}-2 z+2\right) H_{1,3} \\
& -\frac{41}{24}\left(z^{2}-2 z+2\right) H_{1,2,1}+\frac{15}{8}\left(z^{2}-2 z+2\right) H_{2,0,0}+\frac{17}{8}\left(z^{2}-2 z+2\right) H_{4} \\
& -\frac{55}{24}\left(z^{2}-2 z+2\right) H_{1,2,0}-\frac{19}{8}\left(z^{2}-2 z+2\right) H_{1,1,0,0}-\frac{71}{24}\left(z^{2}-2 z+2\right) H_{2,1,0} \\
& +3\left(z^{2}-2 z+2\right) H_{1,0,0,0}-\frac{73}{24}\left(z^{2}-2 z+2\right) H_{2,2}+\frac{25}{8}\left(z^{2}-2 z+2\right) H_{3,0} \\
& -\frac{77}{24}\left(z^{2}-2 z+2\right) H_{2,1,1}-\frac{7}{2}\left(z^{2}-2 z+2\right) H_{1,1,2}-\frac{9}{2}\left(z^{2}-2 z+2\right) H_{0,0,0,0} \\
& -\frac{23}{4}\left(z^{2}-2 z+2\right) H_{1,1,1,0}-\frac{1087}{64}\left(z^{2}-2 z+2\right) \zeta_{4}-\frac{193}{6}\left(z^{2}-2 z+2\right) H_{1,1,1,1} \\
& +\frac{1}{24}\left(20 z^{2}-9 z+19\right) H_{3}+\frac{1}{48}\left(37 z^{2}+622 z+323\right) H_{1,1,1} \\
& +\frac{1}{48}\left(39 z^{2}+20 z+30\right) H_{1,0,0}+\frac{1}{48}\left(106 z^{2}-139 z+282\right) H_{0,0} \\
& +\frac{1}{432}\left(4181 z^{2}-8590 z+6943\right) H_{1,1}+\frac{15026 z^{2}-47650 z+27967}{2592} H_{1} \\
& +\frac{1}{96}\left(-443 z^{2}-502 z-704\right) \zeta_{2} H_{1}-\frac{7}{6}\left(z^{2}-2 z+2\right) \zeta_{3} H_{0} \\
& -\frac{323}{48}\left(z^{2}-2 z+2\right) \zeta_{2} H_{2}-\frac{335}{48}\left(z^{2}-2 z+2\right) \zeta_{2} H_{1,0}+\frac{235}{24}\left(z^{2}-2 z+2\right) \zeta_{3} H_{1} \\
& +\frac{29}{2}\left(z^{2}-2 z+2\right) \zeta_{2} H_{0,0}+20\left(z^{2}-2 z+2\right) \zeta_{2} H_{1,1} \\
& +\frac{1}{96}\left(135 z^{2}+62 z+766\right) \zeta_{2} H_{0} \\
& B_{g q}^{(-1)}=\frac{1}{36}\left(-150 z^{2}+394 z-241\right) H_{1,1}+\frac{1}{36}\left(-13 z^{2}+20 z-2\right) H_{1,0} \\
& +\frac{1}{6}\left(-7 z^{2}+13 z-8\right) H_{0,0}+\frac{1}{12}\left(-z^{2}+2 z-2\right) H_{1,2}+\frac{1}{4}\left(-z^{2}+2 z-2\right) H_{2,1} \\
& -\frac{5}{12}\left(z^{2}-2 z+2\right) H_{1,0,0}-\frac{5}{12}\left(z^{2}-2 z+2\right) H_{1,1,0}-\frac{7}{12}\left(z^{2}-2 z+2\right) H_{2,0} \\
& +\frac{3}{4}\left(z^{2}-2 z+2\right) H_{0,0,0}-\frac{16}{3}\left(z^{2}-2 z+2\right) H_{1,1,1}-\frac{15}{8}\left(z^{2}-2 z+2\right) \zeta_{2} H_{0} \\
& +\frac{193}{48}\left(z^{2}-2 z+2\right) \zeta_{2} H_{1}+\frac{1}{648}\left(-613 z^{2}+2654 z-845\right) H_{1} \\
& +\frac{1}{216}\left(-97 z^{2}+80 z-62\right) H_{0}+\frac{1}{18}\left(-11 z^{2}+7 z+8\right) H_{2}+\frac{1}{4}\left(-z^{2}+2 z-2\right) H_{3} \\
& +\frac{89}{36}\left(z^{2}-2 z+2\right) \zeta_{3}+\frac{1}{96}\left(534 z^{2}-1280 z+903\right) \zeta_{2}+\frac{15476 z^{2}-25644 z+27525}{5184}, \\
& C_{g q}^{(-1)}=-\frac{8}{27}\left(z^{2}-2 z+2\right) H_{1,1}+\frac{1}{81}\left(-47 z^{2}+106 z-79\right) H_{1} \\
& +\frac{1}{12}\left(z^{2}-2 z+2\right) \zeta_{2}+\frac{1}{324}\left(-261 z^{2}+610 z-403\right),
\end{aligned}
$$




$$
\begin{aligned}
& D_{g q}^{(-1)}=\frac{1}{4}\left(z^{2}-2 z+2\right) H_{2,1}+\frac{1}{4}\left(z^{2}-2 z+2\right) H_{1,2}+\frac{5}{12}\left(z^{2}-2 z+2\right) H_{1,1,0} \\
& +\frac{7}{12}\left(z^{2}-2 z+2\right) H_{2,0}+\frac{7}{12}\left(z^{2}-2 z+2\right) H_{1,0,0}-\frac{3}{4}\left(z^{2}-2 z+2\right) H_{0,0,0} \\
& +\frac{68}{9}\left(z^{2}-2 z+2\right) H_{1,1,1}+\frac{1}{36}\left(13 z^{2}-20 z+2\right) H_{1,0}+\frac{1}{12}\left(17 z^{2}-28 z+16\right) H_{0,0} \\
& +\frac{1}{108}\left(928 z^{2}-2258 z+1529\right) H_{1,1}+\frac{15}{8}\left(z^{2}-2 z+2\right) \zeta_{2} H_{0} \\
& -\frac{685}{144}\left(z^{2}-2 z+2\right) \zeta_{2} H_{1}+\frac{1}{4}\left(z^{2}-2 z+2\right) H_{3}+\frac{1}{36}\left(31 z^{2}-20 z-16\right) H_{2} \\
& +\frac{1}{108}\left(53 z^{2}-58 z+58\right) H_{0}+\frac{1}{648}\left(4493 z^{2}-11812 z+7033\right) H_{1} \\
& +\frac{-11975 z^{2}+27682 z-19501}{1728} \zeta_{2}-\frac{325}{144}\left(z^{2}-2 z+2\right) \zeta_{3} \\
& +\frac{30122 z^{2}-89308 z+35497}{7776} \\
& E_{g q}^{(-1)}=\frac{-18400 z^{2}+44972 z+190765}{62208}+\frac{-32990 z^{2}+103120 z-18259}{3456} \zeta_{2} \\
& +\frac{-4079 z^{2}+9530 z-8549}{1728} H_{1}+\frac{1}{288}\left(-2428 z^{2}+6980 z-2759\right) \zeta_{3} \\
& +\frac{1}{16}\left(-61 z^{2}+86 z+28\right) H_{0,0,0}+\frac{1}{48}\left(-28 z^{2}-86 z+11\right) H_{1,0,0} \\
& +\frac{1}{48}\left(-21 z^{2}-16 z-8\right) H_{3}-\frac{7}{12}\left(z^{2}-2 z+2\right) H_{3,1}+\frac{31}{24}\left(z^{2}-2 z+2\right) H_{1,3} \\
& -\frac{9}{4}\left(z^{2}-2 z+2\right) H_{2,0,0}-\frac{5}{2}\left(z^{2}-2 z+2\right) H_{4}+\frac{29}{8}\left(z^{2}-2 z+2\right) H_{1,1,0,0} \\
& +\frac{89}{24}\left(z^{2}-2 z+2\right) H_{1,2,0}+\frac{49}{12}\left(z^{2}-2 z+2\right) H_{2,1,0}+\frac{25}{6}\left(z^{2}-2 z+2\right) H_{2,2} \\
& -\frac{17}{4}\left(z^{2}-2 z+2\right) H_{3,0}+\frac{103}{24}\left(z^{2}-2 z+2\right) H_{1,2,1}-\frac{37}{8}\left(z^{2}-2 z+2\right) H_{1,0,0,0} \\
& +\frac{31}{6}\left(z^{2}-2 z+2\right) H_{2,1,1}+\frac{45}{8}\left(z^{2}-2 z+2\right) H_{0,0,0,0}+\frac{143}{24}\left(z^{2}-2 z+2\right) H_{1,1,2} \\
& +\frac{26}{3}\left(z^{2}-2 z+2\right) H_{1,1,1,0}+\frac{2681}{128}\left(z^{2}-2 z+2\right) \zeta_{4}+\frac{173}{3}\left(z^{2}-2 z+2\right) H_{1,1,1,1} \\
& +\frac{1}{48}\left(52 z^{2}-177 z-204\right) H_{0,0}+\frac{1}{12}\left(82 z^{2}-180 z+81\right) H_{1,1,0} \\
& +\frac{1}{432}\left(151 z^{2}+379 z-1000\right) H_{0}+\frac{1}{48}\left(167 z^{2}-236 z-40\right) H_{2,0} \\
& +\frac{1}{48}\left(234 z^{2}-482 z+209\right) H_{1,2}+\frac{1}{288}\left(511 z^{2}-3952 z+1117\right) H_{1,1} \\
& +\frac{1}{144}\left(533 z^{2}-1363 z+922\right) H_{1,0}+\frac{1}{144}\left(560 z^{2}-1363 z+184\right) H_{2} \\
& +\frac{1}{48}\left(585 z^{2}-602 z-40\right) H_{2,1}+\frac{1}{36}\left(772 z^{2}-2492 z+1007\right) H_{1,1,1} \\
& +\frac{1}{288}\left(-2554 z^{2}+11360 z-4421\right) \zeta_{2} H_{1}+\frac{11}{8}\left(z^{2}-2 z+2\right) \zeta_{3} H_{0} \\
& +\frac{23}{3}\left(z^{2}-2 z+2\right) \zeta_{2} H_{2}+\frac{241}{24}\left(z^{2}-2 z+2\right) \zeta_{2} H_{1,0}-\frac{265}{16}\left(z^{2}-2 z+2\right) \zeta_{2} H_{0,0} \\
& -\frac{141}{8}\left(z^{2}-2 z+2\right) \zeta_{3} H_{1}-\frac{401}{12}\left(z^{2}-2 z+2\right) \zeta_{2} H_{1,1} \\
& +\frac{1}{48}\left(243 z^{2}-430 z-218\right) \zeta_{2} H_{0} \text {, }
\end{aligned}
$$




$$
\begin{aligned}
& F_{g q}^{(-1)}=\frac{8}{27}\left(z^{2}-2 z+2\right) H_{1,1}+\frac{1}{81}\left(47 z^{2}-106 z+79\right) H_{1} \\
& +\frac{1}{12}\left(-z^{2}+2 z-2\right) \zeta_{2}+\frac{1}{324}\left(261 z^{2}-610 z+403\right) \text {, } \\
& G_{g q}^{(-1)}=\frac{-980846 z^{2}+2724232 z-1639885}{62208}+\frac{-49097 z^{2}+130744 z-78310}{2592} H_{1} \\
& +\frac{1}{216}\left(-5257 z^{2}+13928 z-8933\right) H_{1,1}+\frac{1}{144}\left(-4639 z^{2}+11366 z-7397\right) H_{1,1,1} \\
& +\frac{1}{48}\left(-375 z^{2}+374 z-50\right) H_{2,1}+\frac{1}{48}\left(-200 z^{2}+355 z-78\right) H_{0,0} \\
& +\frac{1}{48}\left(-183 z^{2}+230 z-90\right) H_{2,0}+\frac{1}{48}\left(-165 z^{2}+358 z-214\right) H_{1,1,0} \\
& +\frac{1}{48}\left(-148 z^{2}+231 z+26\right) H_{2}+\frac{1}{48}\left(-89 z^{2}+218 z-112\right) H_{1,2} \\
& +\frac{1}{24}\left(-42 z^{2}+105 z-59\right) H_{1,0}+\frac{1}{48}\left(-29 z^{2}+114 z-80\right) H_{1,0,0} \\
& +\frac{1}{24}\left(-14 z^{2}+20 z-15\right) H_{3}+\frac{1}{3}\left(-z^{2}+2 z-2\right) H_{1,3}+\frac{3}{8}\left(z^{2}-2 z+2\right) H_{4} \\
& +\frac{3}{8}\left(z^{2}-2 z+2\right) H_{2,0,0}+\frac{5}{8}\left(z^{2}-2 z+2\right) H_{3,1}-\frac{9}{8}\left(z^{2}-2 z+2\right) H_{0,0,0,0} \\
& +\frac{9}{8}\left(z^{2}-2 z+2\right) H_{3,0}-\frac{9}{8}\left(z^{2}-2 z+2\right) H_{2,2}-\frac{9}{8}\left(z^{2}-2 z+2\right) H_{2,1,0} \\
& -\frac{13}{8}\left(z^{2}-2 z+2\right) H_{1,1,0,0}-\frac{43}{24}\left(z^{2}-2 z+2\right) H_{1,2,0}-\frac{47}{24}\left(z^{2}-2 z+2\right) H_{2,1,1} \\
& +2\left(z^{2}-2 z+2\right) H_{1,0,0,0}-\frac{17}{6}\left(z^{2}-2 z+2\right) H_{1,1,2}-\frac{35}{12}\left(z^{2}-2 z+2\right) H_{1,1,1,0} \\
& -\frac{83}{24}\left(z^{2}-2 z+2\right) H_{1,2,1}-\frac{3739}{768}\left(z^{2}-2 z+2\right) \zeta_{4}-\frac{185}{6}\left(z^{2}-2 z+2\right) H_{1,1,1,1} \\
& +\frac{1}{48}\left(3 z^{2}-75 z+94\right) H_{0}+\frac{1}{24}\left(106 z^{2}-132 z+45\right) H_{0,0,0} \\
& +\frac{1}{288}\left(3557 z^{2}-7954 z+5056\right) \zeta_{3}+\frac{83390 z^{2}-192112 z+109609}{3456} \zeta_{2} \\
& +\frac{1}{32}\left(-225 z^{2}+278 z-110\right) \zeta_{2} H_{0}-\frac{5}{24}\left(z^{2}-2 z+2\right) \zeta_{3} H_{0} \\
& -\frac{15}{16}\left(z^{2}-2 z+2\right) \zeta_{2} H_{2}+\frac{33}{16}\left(z^{2}-2 z+2\right) \zeta_{2} H_{0,0}-\frac{55}{16}\left(z^{2}-2 z+2\right) \zeta_{2} H_{1,0} \\
& +\frac{77}{8}\left(z^{2}-2 z+2\right) \zeta_{3} H_{1}+\frac{367}{24}\left(z^{2}-2 z+2\right) \zeta_{2} H_{1,1} \\
& +\frac{1}{288}\left(4729 z^{2}-11834 z+7973\right) \zeta_{2} H_{1} \text {, } \\
& H_{g q}^{(-1)}=\frac{1}{54}\left(-239 z^{2}+538 z-403\right) H_{1,1}+\frac{1}{6}\left(-z^{2}+2 z-2\right) H_{1,0,0} \\
& +\frac{1}{6}\left(-z^{2}+2 z-2\right) H_{1,2}-\frac{20}{9}\left(z^{2}-2 z+2\right) H_{1,1,1}-\frac{1}{12} z(3 z-2) H_{0,0} \\
& +\frac{53}{72}\left(z^{2}-2 z+2\right) \zeta_{2} H_{1}+\frac{1}{324}\left(-1940 z^{2}+4579 z-3094\right) H_{1} \\
& +\frac{1}{24}\left(-z^{2}+4 z-6\right) H_{0}-\frac{1}{12} z(3 z-2) H_{2}-\frac{31}{144}\left(z^{2}-2 z+2\right) \zeta_{3} \\
& +\frac{2363 z^{2}-4642 z+3247}{1728} \zeta_{2}+\frac{-106672 z^{2}+255548 z-153569}{15552}, \\
& I_{g q}^{(-1)}=\frac{3}{8}\left(z^{2}-2 z+2\right) \zeta_{2} H_{1,0}-\frac{15}{8}\left(z^{2}-2 z+2\right) \zeta_{2} H_{1,1}
\end{aligned}
$$




$$
\begin{aligned}
& -\frac{3}{8}\left(z^{2}-2 z+2\right) H_{1,0,0,0}+\frac{3}{8}\left(z^{2}-2 z+2\right) H_{1,2,0}+\frac{3}{8}\left(z^{2}-2 z+2\right) H_{1,1,0,0} \\
& +\frac{3}{8}\left(z^{2}-2 z+2\right) H_{1,1,2}+\frac{7}{8}\left(z^{2}-2 z+2\right) H_{1,2,1}+\frac{16}{3}\left(z^{2}-2 z+2\right) H_{1,1,1,1} \\
& +\frac{1}{16}\left(6 z^{2}-16 z+13\right) H_{1,0,0}+\frac{1}{16}\left(6 z^{2}-16 z+13\right) H_{1,2} \\
& +\frac{2}{3}\left(15 z^{2}-34 z+25\right) H_{1,1,1}+\frac{1}{96}\left(1237 z^{2}-2964 z+2055\right) H_{1,1} \\
& +\frac{1}{8}\left(z^{2}-2 z+2\right) H_{1,3}+\frac{3}{16}(1-z) z H_{1,0}-\frac{3}{16} z(3 z-2) H_{0,0,0} \\
& +\frac{3}{16} z(3 z-2) H_{2,0}+\frac{7}{16} z(3 z-2) H_{2,1}+\frac{1}{16} z(14 z-13) H_{0,0} \\
& +\frac{1}{16}\left(-47 z^{2}+110 z-80\right) \zeta_{2} H_{1}-\frac{43}{24}\left(z^{2}-2 z+2\right) \zeta_{3} H_{1} \\
& +\frac{1}{192}\left(2977 z^{2}-7214 z+4679\right) H_{1}+\frac{3}{16} z(3 z-2) \zeta_{2} H_{0}+\frac{5}{16}(1-z) z H_{0} \\
& +\frac{1}{16} z(3 z-2) H_{3}+\frac{1}{16} z(10 z-9) H_{2}+\frac{1}{384}\left(-1729 z^{2}+3554 z-2102\right) \zeta_{2} \\
& +\frac{1}{96}\left(-339 z^{2}+698 z-485\right) \zeta_{3}+\frac{697}{768}\left(z^{2}-2 z+2\right) \zeta_{4} \\
& +\frac{1}{768}\left(13880 z^{2}-33564 z+20835\right) \text {, } \\
& A_{g q}^{(0)}=\frac{-16397336 z^{2}+30450460 z-25185649}{373248} \\
& +\frac{1}{576}\left(-5541 z^{2}+10102 z-8938\right) \zeta_{3}+\frac{1}{288}\left(-1286 z^{2}+4288 z-3079\right) H_{1,2} \\
& +\frac{1}{48}\left(-1177 z^{2}+1174 z+686\right) H_{2,1,1}+\frac{1}{48}\left(-677 z^{2}+833 z-870\right) H_{0,0,0} \\
& +\frac{1}{48}\left(-621 z^{2}+1398 z-550\right) H_{1,2,1}+\frac{1}{48}\left(-575 z^{2}+1478 z-387\right) H_{1,1,2} \\
& +\frac{1}{48}\left(-546 z^{2}+1642 z-143\right) H_{1,1,1,0}+\frac{1}{48}\left(-219 z^{2}+276 z+302\right) H_{2,1,0} \\
& +\frac{1}{144}\left(-187 z^{2}+9638 z+4753\right) H_{1,1,1,1}+\frac{1}{48}\left(-175 z^{2}+244 z-338\right) H_{2,0,0} \\
& +\frac{1}{48}\left(-122 z^{2}-42 z-197\right) H_{1,0,0,0}+\frac{1}{24}\left(-86 z^{2}+128 z+103\right) H_{2,2} \\
& +\frac{1}{48}\left(-55 z^{2}-8 z-138\right) H_{4}+\frac{1}{24}\left(-54 z^{2}+20 z+135\right) H_{3} \\
& +\frac{1}{48}\left(-31 z^{2}+88 z-462\right) H_{3,0}+\frac{1}{48}\left(-27 z^{2}+276 z-74\right) H_{1,2,0} \\
& +\frac{1}{24}\left(-18 z^{2}+145 z-47\right) H_{1,1,0,0}+\frac{13}{24}\left(z^{2}-2 z+2\right) H_{1,3,1} \\
& +\frac{11}{8}\left(z^{2}-2 z+2\right) H_{4,1}-\frac{13}{8}\left(z^{2}-2 z+2\right) H_{1,1,3}+\frac{19}{8}\left(z^{2}-2 z+2\right) H_{1,2,0,0} \\
& +\frac{61}{24}\left(z^{2}-2 z+2\right) H_{1,4}+\frac{67}{24}\left(z^{2}-2 z+2\right) H_{2,2,0}+\frac{23}{8}\left(z^{2}-2 z+2\right) H_{2,1,0,0} \\
& +\frac{77}{24}\left(z^{2}-2 z+2\right) H_{2,3}-\frac{33}{8}\left(z^{2}-2 z+2\right) H_{2,0,0,0}-\frac{39}{8}\left(z^{2}-2 z+2\right) H_{3,0,0} \\
& -\frac{45}{8}\left(z^{2}-2 z+2\right) H_{5}+\frac{137}{24}\left(z^{2}-2 z+2\right) H_{3,1,1}+\frac{139}{24}\left(z^{2}-2 z+2\right) H_{1,3,0} \\
& +\frac{53}{8}\left(z^{2}-2 z+2\right) H_{3,1,0}-\frac{53}{8}\left(z^{2}-2 z+2\right) H_{1,2,1,0}+\frac{55}{8}\left(z^{2}-2 z+2\right) H_{3,2}
\end{aligned}
$$




$$
\begin{aligned}
& -7\left(z^{2}-2 z+2\right) H_{1,2,2}-\frac{57}{8}\left(z^{2}-2 z+2\right) H_{4,0}-\frac{15}{2}\left(z^{2}-2 z+2\right) H_{1,1,2,0} \\
& -\frac{97}{12}\left(z^{2}-2 z+2\right) H_{1,1,1,0,0}+\frac{49}{6}\left(z^{2}-2 z+2\right) H_{1,1,0,0,0} \\
& -\frac{33}{4}\left(z^{2}-2 z+2\right) H_{1,0,0,0,0}+\frac{42}{5}\left(z^{2}-2 z+2\right) \zeta_{5}-\frac{205}{24}\left(z^{2}-2 z+2\right) H_{2,1,2} \\
& -\frac{211}{24}\left(z^{2}-2 z+2\right) H_{2,2,1}-\frac{227}{24}\left(z^{2}-2 z+2\right) H_{2,1,1,0}+\frac{45}{4}\left(z^{2}-2 z+2\right) H_{0,0,0,0,0} \\
& -\frac{107}{8}\left(z^{2}-2 z+2\right) H_{1,2,1,1}-\frac{497}{24}\left(z^{2}-2 z+2\right) H_{1,1,2,1}-\frac{169}{8}\left(z^{2}-2 z+2\right) H_{2,1,1,1} \\
& -\frac{229}{8}\left(z^{2}-2 z+2\right) H_{1,1,1,2}-\frac{479}{12}\left(z^{2}-2 z+2\right) H_{1,1,1,1,0} \\
& -\frac{635}{4}\left(z^{2}-2 z+2\right) H_{1,1,1,1,1}+\frac{1}{24}\left(4 z^{2}+269 z+149\right) H_{2,1} \\
& +\frac{1}{8}\left(14 z^{2}-22 z+99\right) H_{0,0,0,0}+\frac{1}{48}\left(38 z^{2}+20 z-63\right) H_{1,3} \\
& +\frac{1}{288}\left(74 z^{2}+98 z+673\right) H_{1,0,0}+\frac{1}{48}\left(85 z^{2}+54 z-310\right) H_{3,1} \\
& +\frac{1}{288}\left(89 z^{2}+1358 z-365\right) H_{1,1,0}+\frac{1}{72}\left(190 z^{2}-341 z+971\right) H_{2,0} \\
& +\frac{32851 z^{2}+8134 z-5641}{1152} \zeta_{4}+\frac{1}{864}\left(36545 z^{2}-72694 z+57709\right) H_{1,1,1} \\
& +\frac{-540 z^{3}-91615 z^{2}-20078 z-85789}{10368} H_{1}+\frac{-270 z^{3}+47581 z^{2}-97775 z+97568}{5184} H_{0} \\
& +\frac{-360 z^{4}-1584 z^{3}-82700 z^{2}+147900 z-72535}{2304} \zeta_{2} \\
& +\frac{1}{288}\left(15 z^{4}+66 z^{3}+2498 z^{2}-2009 z+172\right) H_{0,0} \\
& +\frac{1}{864}\left(45 z^{4}+198 z^{3}+7672 z^{2}-4667 z+1028\right) H_{2} \\
& +\frac{90 z^{4}+396 z^{3}+7973 z^{2}-12622 z+8959}{1728} H_{1,0} \\
& +\frac{90 z^{4}+396 z^{3}+42239 z^{2}-128522 z+75557}{1728} H_{1,1} \\
& +\frac{1}{576}\left(-21455 z^{2}+43270 z-38449\right) \zeta_{2} H_{1}+\frac{1}{288}\left(-3095 z^{2}-5414 z-8389\right) \zeta_{2} H_{1,1} \\
& +\frac{1}{144}\left(-659 z^{2}-1514 z-2344\right) \zeta_{3} H_{1}+\frac{1}{8}\left(z^{2}-2 z+2\right) \zeta_{3} H_{1,0} \\
& +\frac{37}{12}\left(z^{2}-2 z+2\right) \zeta_{3} H_{0,0}-\frac{55}{16}\left(z^{2}-2 z+2\right) \zeta_{2} H_{1,2}-\frac{169}{48}\left(z^{2}-2 z+2\right) \zeta_{2} H_{2,1} \\
& -\frac{215}{48}\left(z^{2}-2 z+2\right) \zeta_{2} H_{1,1,0}+\frac{39}{8}\left(z^{2}-2 z+2\right) \zeta_{3} H_{2}+\frac{293}{16}\left(z^{2}-2 z+2\right) \zeta_{2} H_{3} \\
& +\frac{297}{16}\left(z^{2}-2 z+2\right) \zeta_{2} H_{2,0}+\frac{435}{16}\left(z^{2}-2 z+2\right) \zeta_{2} H_{1,0,0}+\frac{5539}{192}\left(z^{2}-2 z+2\right) \zeta_{4} H_{0} \\
& -\frac{11843}{384}\left(z^{2}-2 z+2\right) \zeta_{4} H_{1}-\frac{315}{8}\left(z^{2}-2 z+2\right) \zeta_{2} H_{0,0,0}-\frac{1927}{48}\left(z^{2}-2 z+2\right) \zeta_{2} \zeta_{3} \\
& +\frac{177}{4}\left(z^{2}-2 z+2\right) \zeta_{3} H_{1,1}+\frac{611}{8}\left(z^{2}-2 z+2\right) \zeta_{2} H_{1,1,1} \\
& +\frac{3}{16}\left(17 z^{2}+10 z-50\right) \zeta_{3} H_{0}+\frac{1}{96}\left(881 z^{2}-1266 z-2562\right) \zeta_{2} H_{0,0} \\
& +\frac{1}{48}\left(911 z^{2}-1288 z+2195\right) \zeta_{2} H_{0}+\frac{1}{96}\left(1599 z^{2}-1886 z+1794\right) \zeta_{2} H_{1,0}
\end{aligned}
$$




$$
\begin{aligned}
& +\frac{1}{96}\left(1791 z^{2}-1994 z+1650\right) \zeta_{2} H_{2}, \\
& B_{g q}^{(0)}=\frac{7\left(380164 z^{2}-827612 z+637397\right)}{93312} \\
& +\frac{1}{216}\left(-4207 z^{2}+10718 z-6479\right) H_{1,1,1}+\frac{1}{72}\left(-187 z^{2}+362 z-224\right) H_{2,0} \\
& +\frac{1}{72}\left(-172 z^{2}+338 z-167\right) H_{1,2}+\frac{1}{36}\left(-169 z^{2}+368 z-224\right) H_{1,1,0} \\
& +\frac{1}{24}\left(-121 z^{2}+158 z-32\right) H_{2,1}+\frac{1}{72}\left(-68 z^{2}+178 z-151\right) H_{1,0,0} \\
& +\frac{1}{12}\left(-z^{2}+2 z-2\right) H_{1,3}+\frac{3}{4}\left(z^{2}-2 z+2\right) H_{4}+\frac{3}{4}\left(z^{2}-2 z+2\right) H_{3,1} \\
& -\frac{5}{6}\left(z^{2}-2 z+2\right) H_{1,2,0}-\frac{5}{6}\left(z^{2}-2 z+2\right) H_{1,1,0,0}+\frac{13}{12}\left(z^{2}-2 z+2\right) H_{2,0,0} \\
& -\frac{7}{6}\left(z^{2}-2 z+2\right) H_{1,2,1}-\frac{5}{4}\left(z^{2}-2 z+2\right) H_{2,2}-\frac{17}{12}\left(z^{2}-2 z+2\right) H_{2,1,0} \\
& +\frac{19}{12}\left(z^{2}-2 z+2\right) H_{1,0,0,0}+\frac{7}{4}\left(z^{2}-2 z+2\right) H_{3,0}-\frac{9}{4}\left(z^{2}-2 z+2\right) H_{0,0,0,0} \\
& -\frac{29}{12}\left(z^{2}-2 z+2\right) H_{2,1,1}-\frac{5}{2}\left(z^{2}-2 z+2\right) H_{1,1,2}-\frac{53}{12}\left(z^{2}-2 z+2\right) H_{1,1,1,0} \\
& -\frac{5269\left(z^{2}-2 z+2\right)}{1152} \zeta_{4}-\frac{224}{9}\left(z^{2}-2 z+2\right) H_{1,1,1,1}+\frac{1}{6}\left(2 z^{2}+3 z-8\right) H_{3} \\
& +\frac{1}{6}\left(30 z^{2}-49 z+24\right) H_{0,0,0}+\frac{1}{432}\left(3923 z^{2}-9802 z+6310\right) \zeta_{3} \\
& +\frac{27 z^{3}-6409 z^{2}+12065 z-10778}{1296} H_{0}+\frac{54 z^{3}+31507 z^{2}-58986 z+52929}{2592} H_{1} \\
& +\frac{-27 z^{4}-6604 z^{2}+22910 z-7547}{1296} H_{1,1}+\frac{1}{432}\left(-9 z^{4}-2552 z^{2}+2938 z-124\right) H_{2} \\
& +\frac{1}{432}\left(-9 z^{4}-2012 z^{2}+3994 z-2401\right) H_{1,0} \\
& +\frac{1}{144}\left(-3 z^{4}-592 z^{2}+766 z+124\right) H_{0,0}+\frac{1}{576}\left(36 z^{4}+9511 z^{2}-22666 z+10514\right) \zeta_{2} \\
& +\frac{1}{12}\left(-108 z^{2}+197 z-120\right) \zeta_{2} H_{0}-\frac{5}{12}\left(z^{2}-2 z+2\right) \zeta_{3} H_{0} \\
& -\frac{73}{24}\left(z^{2}-2 z+2\right) \zeta_{2} H_{2}-\frac{79}{24}\left(z^{2}-2 z+2\right) \zeta_{2} H_{1,0}+\frac{45}{8}\left(z^{2}-2 z+2\right) \zeta_{2} H_{0,0} \\
& +\frac{691}{72}\left(z^{2}-2 z+2\right) \zeta_{3} H_{1}+\frac{1007}{72}\left(z^{2}-2 z+2\right) \zeta_{2} H_{1,1} \\
& +\frac{1}{288}\left(4399 z^{2}-11042 z+7757\right) \zeta_{2} H_{1}, \\
& C_{g q}^{(0)}=-\frac{32}{27}\left(z^{2}-2 z+2\right) H_{1,1,1}-\frac{4}{81}\left(47 z^{2}-106 z+79\right) H_{1,1} \\
& +\frac{1}{3}\left(z^{2}-2 z+2\right) \zeta_{2} H_{1}+\frac{1}{81}\left(-261 z^{2}+610 z-403\right) H_{1}+\frac{17}{54}\left(z^{2}-2 z+2\right) \zeta_{3} \\
& +\frac{1}{72}\left(47 z^{2}-106 z+79\right) \zeta_{2}+\frac{-11287 z^{2}+26714 z-16643}{2916}, \\
& D_{g q}^{(0)}=\frac{-7127 z^{2}-90310 z-142088}{15552}+\frac{1}{288}\left(-2899 z^{2}+6834 z-4065\right) \zeta_{3} \\
& +\frac{1}{4}\left(-25 z^{2}+36 z-16\right) H_{0,0,0}+\frac{1}{12}\left(-7 z^{2}-4 z+16\right) H_{3}+\frac{1}{12}\left(-z^{2}+2 z-2\right) H_{1,3} \\
& -\frac{3}{4}\left(z^{2}-2 z+2\right) H_{4}-\frac{3}{4}\left(z^{2}-2 z+2\right) H_{3,1}-\frac{13}{12}\left(z^{2}-2 z+2\right) H_{2,0,0}
\end{aligned}
$$




$$
\begin{aligned}
& +\frac{13}{12}\left(z^{2}-2 z+2\right) H_{1,2,0}+\frac{5}{4}\left(z^{2}-2 z+2\right) H_{2,2}+\frac{5}{4}\left(z^{2}-2 z+2\right) H_{1,1,0,0} \\
& +\frac{17}{12}\left(z^{2}-2 z+2\right) H_{2,1,0}-\frac{7}{4}\left(z^{2}-2 z+2\right) H_{3,0}+\frac{25}{12}\left(z^{2}-2 z+2\right) H_{1,2,1} \\
& +\frac{9}{4}\left(z^{2}-2 z+2\right) H_{0,0,0,0}+\frac{29}{12}\left(z^{2}-2 z+2\right) H_{2,1,1}-\frac{29}{12}\left(z^{2}-2 z+2\right) H_{1,0,0,0} \\
& +\frac{35}{12}\left(z^{2}-2 z+2\right) H_{1,1,2}+\frac{53}{12}\left(z^{2}-2 z+2\right) H_{1,1,1,0}+\frac{19127\left(z^{2}-2 z+2\right)}{2304} \zeta_{4} \\
& +\frac{304}{9}\left(z^{2}-2 z+2\right) H_{1,1,1,1}+\frac{1}{24}\left(67 z^{2}-140 z+83\right) H_{1,2} \\
& +\frac{1}{12}\left(77 z^{2}-90 z+16\right) H_{2,1}+\frac{1}{72}\left(97 z^{2}-260 z+233\right) H_{1,0,0} \\
& +\frac{1}{36}\left(107 z^{2}-190 z+112\right) H_{2,0}+\frac{1}{36}\left(169 z^{2}-368 z+224\right) H_{1,1,0} \\
& +\frac{1}{72}\left(2677 z^{2}-6442 z+4309\right) H_{1,1,1}+\frac{-162 z^{3}+114881 z^{2}-332356 z+161365}{7776} H_{1} \\
& +\frac{-27 z^{3}+7660 z^{2}-15773 z+15044}{1296} H_{0}+\frac{-324 z^{4}-127855 z^{2}+288266 z-139847}{5184} \zeta_{2} \\
& +\frac{1}{144}\left(3 z^{4}+763 z^{2}-842 z-232\right) H_{0,0}+\frac{1}{432}\left(9 z^{4}+2156 z^{2}-4336 z+2707\right) H_{1,0} \\
& +\frac{1}{432}\left(9 z^{4}+3137 z^{2}-3454 z+232\right) H_{2} \\
& +\frac{1}{432}\left(9 z^{4}+12467 z^{2}-31950 z+19098\right) H_{1,1} \\
& +\frac{1}{864}\left(-17407 z^{2}+42818 z-30749\right) \zeta_{2} H_{1}-\frac{7}{12}\left(z^{2}-2 z+2\right) \zeta_{3} H_{0} \\
& +\frac{73}{24}\left(z^{2}-2 z+2\right) \zeta_{2} H_{2}+\frac{113}{24}\left(z^{2}-2 z+2\right) \zeta_{2} H_{1,0}-\frac{45}{8}\left(z^{2}-2 z+2\right) \zeta_{2} H_{0,0} \\
& -\frac{737}{72}\left(z^{2}-2 z+2\right) \zeta_{3} H_{1}-\frac{1201}{72}\left(z^{2}-2 z+2\right) \zeta_{2} H_{1,1} \\
& +\frac{1}{24}\left(267 z^{2}-428 z+240\right) \zeta_{2} H_{0} \\
& E_{g q}^{(0)}=\frac{-556382 z^{2}+5813728 z+1746191}{373248}+\frac{1}{48}(31 z-36)(91 z+6) H_{2,1,1} \\
& +\frac{-365539 z^{2}+164798 z-12626}{4608} \zeta_{4}+\frac{-15752 z^{2}+63484 z-25099}{1728} \zeta_{3} \\
& +\frac{1}{48}\left(-549 z^{2}+530 z+160\right) H_{3,1}+\frac{1}{16}\left(-167 z^{2}+208 z+64\right) H_{3,0} \\
& +\frac{1}{8}\left(-21 z^{2}+39 z+8\right) H_{4}+\frac{1}{48}\left(-20 z^{2}-60 z+147\right) H_{1,3} \\
& +\frac{1}{48}\left(z^{2}-32 z+248\right) H_{2,0,0}+\frac{1}{2}\left(z^{2}-2 z+2\right) H_{4,1}+\frac{13}{12}\left(z^{2}-2 z+2\right) H_{1,1,3} \\
& -\frac{47}{12}\left(z^{2}-2 z+2\right) H_{2,2,0}-\frac{47}{12}\left(z^{2}-2 z+2\right) H_{1,2,0,0}-4\left(z^{2}-2 z+2\right) H_{2,1,0,0} \\
& -\frac{101}{24}\left(z^{2}-2 z+2\right) H_{1,3,1}-\frac{17}{4}\left(z^{2}-2 z+2\right) H_{1,4}-\frac{13}{3}\left(z^{2}-2 z+2\right) H_{2,3} \\
& +\frac{9}{2}\left(z^{2}-2 z+2\right) H_{2,0,0,0}+6\left(z^{2}-2 z+2\right) H_{3,0,0}+\frac{27}{4}\left(z^{2}-2 z+2\right) H_{5} \\
& -10\left(z^{2}-2 z+2\right) H_{3,1,0}-\frac{81}{8}\left(z^{2}-2 z+2\right) H_{1,3,0}-\frac{41}{4}\left(z^{2}-2 z+2\right) H_{3,2} \\
& +\frac{21}{2}\left(z^{2}-2 z+2\right) H_{4,0}+\frac{85}{8}\left(z^{2}-2 z+2\right) H_{1,2,2}+\frac{65}{6}\left(z^{2}-2 z+2\right) H_{1,2,1,0}
\end{aligned}
$$




$$
\begin{aligned}
& +\frac{133}{12}\left(z^{2}-2 z+2\right) H_{1,1,1,0,0}-\frac{139}{12}\left(z^{2}-2 z+2\right) H_{3,1,1}+\frac{93}{8}\left(z^{2}-2 z+2\right) H_{1,1,2,0} \\
& +\frac{143}{12}\left(z^{2}-2 z+2\right) H_{2,1,2}+\frac{73}{6}\left(z^{2}-2 z+2\right) H_{2,2,1}+\frac{51}{4}\left(z^{2}-2 z+2\right) H_{1,0,0,0,0} \\
& +\frac{77}{6}\left(z^{2}-2 z+2\right) H_{2,1,1,0}-\frac{105}{8}\left(z^{2}-2 z+2\right) H_{1,1,0,0,0} \\
& -\frac{117}{8}\left(z^{2}-2 z+2\right) H_{0,0,0,0,0}-\frac{1937}{120}\left(z^{2}-2 z+2\right) \zeta_{5}+\frac{647}{24}\left(z^{2}-2 z+2\right) H_{1,2,1,1} \\
& +\frac{97}{3}\left(z^{2}-2 z+2\right) H_{2,1,1,1}+\frac{205}{6}\left(z^{2}-2 z+2\right) H_{1,1,2,1}+\frac{175}{4}\left(z^{2}-2 z+2\right) H_{1,1,1,2} \\
& +\frac{176}{3}\left(z^{2}-2 z+2\right) H_{1,1,1,1,0}+\frac{830}{3}\left(z^{2}-2 z+2\right) H_{1,1,1,1,1} \\
& +\frac{1}{16}\left(19 z^{2}-144 z+64\right) H_{1,1,0,0}+\frac{3}{8}\left(25 z^{2}-34 z-18\right) H_{0,0,0,0} \\
& +\frac{1}{24}\left(58 z^{2}-297 z+150\right) H_{1,2,0}+\frac{1}{48}\left(125 z^{2}+188 z+166\right) H_{1,0,0,0} \\
& +\frac{1}{48}\left(247 z^{2}-196 z-192\right) H_{3}+\frac{1}{96}\left(517 z^{2}+426 z+1272\right) H_{0,0,0} \\
& +\frac{1}{48}\left(591 z^{2}-862 z+64\right) H_{2,2}+\frac{1}{48}\left(679 z^{2}-916 z-32\right) H_{2,1,0} \\
& +\frac{1}{24}\left(815 z^{2}-2043 z+858\right) H_{1,1,1,0}+\frac{1}{48}\left(923 z^{2}-2293 z-168\right) H_{2,1} \\
& +\frac{1}{288}\left(950 z^{2}-2746 z+823\right) H_{1,0,0}+\frac{1}{48}\left(965 z^{2}-2318 z+1019\right) H_{1,2,1} \\
& +\frac{1}{48}\left(1267 z^{2}-3082 z+1360\right) H_{1,1,2}+\frac{1}{96}\left(1507 z^{2}-4052 z+2466\right) H_{1,2} \\
& +\frac{1}{18}\left(1912 z^{2}-5900 z+2309\right) H_{1,1,1,1}+\frac{1}{288}\left(2375 z^{2}-4576 z-2480\right) H_{2,0} \\
& +\frac{1}{144}\left(2702 z^{2}-7429 z+4351\right) H_{1,1,0}+\frac{1}{432}\left(4384 z^{2}-29357 z+10241\right) H_{1,1,1} \\
& +\frac{243 z^{3}-29689 z^{2}+105344 z-132128}{5184} H_{0} \\
& +\frac{729 z^{3}-239143 z^{2}+815777 z-506546}{15552} H_{1} \\
& +\frac{-243 z^{4}-1836 z^{3}-66500 z^{2}+169756 z-153745}{5184} H_{1,1} \\
& +\frac{-81 z^{4}-612 z^{3}+8155 z^{2}-32108 z-5440}{1728} H_{2} \\
& +\frac{-81 z^{4}-612 z^{3}+16075 z^{2}-39092 z+17726}{1728} H_{1,0} \\
& +\frac{1}{576}\left(-27 z^{4}-204 z^{3}+2075 z^{2}-12478 z+3040\right) H_{0,0} \\
& +\frac{2916 z^{4}+22032 z^{3}-706466 z^{2}+2354032 z-1361215}{20736} \zeta_{2} \\
& +\frac{1}{864}\left(-17312 z^{2}+61927 z-22048\right) \zeta_{2} H_{1}+\frac{1}{144}\left(-6283 z^{2}+22316 z-8663\right) \zeta_{2} H_{1,1} \\
& +\frac{1}{144}\left(-3971 z^{2}+13054 z-4810\right) \zeta_{3} H_{1}+\frac{1}{96}\left(-1291 z^{2}+1254 z-1200\right) \zeta_{2} H_{2} \\
& +\frac{1}{32}\left(-1049 z^{2}+1374 z+524\right) \zeta_{2} H_{0,0}+\frac{1}{48}\left(-847 z^{2}+772 z-865\right) \zeta_{2} H_{1,0} \\
& +\frac{1}{96}\left(-96 z^{2}-783 z-3532\right) \zeta_{2} H_{0}+\frac{1}{12}\left(-92 z^{2}+4 z+125\right) \zeta_{3} H_{0}
\end{aligned}
$$




$$
\begin{aligned}
& -\frac{43}{24}\left(z^{2}-2 z+2\right) \zeta_{3} H_{1,0}-\frac{89}{24}\left(z^{2}-2 z+2\right) \zeta_{3} H_{0,0}+\frac{29}{6}\left(z^{2}-2 z+2\right) \zeta_{2} H_{2,1} \\
& +\frac{307}{48}\left(z^{2}-2 z+2\right) \zeta_{2} H_{1,2}-\frac{77}{12}\left(z^{2}-2 z+2\right) \zeta_{3} H_{2}+\frac{265}{24}\left(z^{2}-2 z+2\right) \zeta_{2} H_{1,1,0} \\
& -\frac{7463}{384}\left(z^{2}-2 z+2\right) \zeta_{4} H_{0}-21\left(z^{2}-2 z+2\right) \zeta_{2} H_{2,0}-\frac{169}{8}\left(z^{2}-2 z+2\right) \zeta_{2} H_{3} \\
& +\frac{10901}{384}\left(z^{2}-2 z+2\right) \zeta_{4} H_{1}-\frac{607}{16}\left(z^{2}-2 z+2\right) \zeta_{2} H_{1,0,0} \\
& +\frac{729}{16}\left(z^{2}-2 z+2\right) \zeta_{2} H_{0,0,0}+\frac{2057}{32}\left(z^{2}-2 z+2\right) \zeta_{2} \zeta_{3}-\frac{943}{12}\left(z^{2}-2 z+2\right) \zeta_{3} H_{1,1} \\
& -\frac{785}{6}\left(z^{2}-2 z+2\right) \zeta_{2} H_{1,1,1}, \\
& F_{g q}^{(0)}=\frac{32}{27}\left(z^{2}-2 z+2\right) H_{1,1,1}+\frac{4}{81}\left(47 z^{2}-106 z+79\right) H_{1,1} \\
& +\frac{1}{3}\left(-z^{2}+2 z-2\right) \zeta_{2} H_{1}+\frac{1}{81}\left(261 z^{2}-610 z+403\right) H_{1} \\
& +\frac{1}{72}\left(-47 z^{2}+106 z-79\right) \zeta_{2}-\frac{17}{54}\left(z^{2}-2 z+2\right) \zeta_{3}+\frac{11287 z^{2}-26714 z+16643}{2916}, \\
& G_{g q}^{(0)}=\frac{-9858416 z^{2}+29314708 z-16722367}{373248} \\
& +\frac{1}{864}\left(-86929 z^{2}+232766 z-149741\right) H_{1,1,1} \\
& +\frac{1}{144}\left(-20869 z^{2}+50618 z-32825\right) H_{1,1,1,1}+\frac{1}{288}\left(-3136 z^{2}+8192 z-4571\right) H_{1,2} \\
& +\frac{1}{48}\left(-2031 z^{2}+2174 z-470\right) H_{2,1,1}+\frac{1}{96}\left(-1603 z^{2}+4134 z-2641\right) H_{1,1,0} \\
& +\frac{1}{48}\left(-1084 z^{2}+2444 z-1573\right) H_{1,1,1,0}+\frac{1}{288}\left(-1024 z^{2}+3062 z-1739\right) H_{1,0,0} \\
& +\frac{1}{48}\left(-755 z^{2}+1766 z-1099\right) H_{1,1,2}+\frac{1}{48}\left(-613 z^{2}+730 z-270\right) H_{2,1,0} \\
& +\frac{1}{24}\left(-566 z^{2}+969 z-65\right) H_{2,1}+\frac{1}{48}\left(-443 z^{2}+1202 z-706\right) H_{1,2,1} \\
& +\frac{1}{24}\left(-307 z^{2}+537 z-117\right) H_{2,0}+\frac{1}{24}\left(-259 z^{2}+336 z-135\right) H_{2,2} \\
& +\frac{1}{48}\left(-175 z^{2}+198 z-78\right) H_{3}+\frac{1}{48}\left(-125 z^{2}+426 z-316\right) H_{1,2,0} \\
& +\frac{1}{8}\left(-98 z^{2}+130 z-45\right) H_{0,0,0,0}+\frac{1}{12}\left(-21 z^{2}+76 z-56\right) H_{1,1,0,0} \\
& +\frac{1}{48}\left(-18 z^{2}+52 z-105\right) H_{1,3}+\frac{7}{24}\left(z^{2}-2 z+2\right) H_{1,1,3}-\frac{3}{8}\left(z^{2}-2 z+2\right) H_{2,0,0,0} \\
& -\frac{9}{8}\left(z^{2}-2 z+2\right) H_{5}-\frac{9}{8}\left(z^{2}-2 z+2\right) H_{3,0,0}+\frac{9}{8}\left(z^{2}-2 z+2\right) H_{2,3} \\
& +\frac{9}{8}\left(z^{2}-2 z+2\right) H_{2,2,0}+\frac{9}{8}\left(z^{2}-2 z+2\right) H_{2,1,0,0}+\frac{37}{24}\left(z^{2}-2 z+2\right) H_{1,2,0,0} \\
& -\frac{15}{8}\left(z^{2}-2 z+2\right) H_{4,1}+\frac{59}{24}\left(z^{2}-2 z+2\right) H_{1,4}+\frac{27}{8}\left(z^{2}-2 z+2\right) H_{0,0,0,0,0} \\
& -\frac{27}{8}\left(z^{2}-2 z+2\right) H_{4,0}+\frac{27}{8}\left(z^{2}-2 z+2\right) H_{3,2}+\frac{27}{8}\left(z^{2}-2 z+2\right) H_{3,1,0} \\
& -\frac{27}{8}\left(z^{2}-2 z+2\right) H_{2,2,1}-\frac{27}{8}\left(z^{2}-2 z+2\right) H_{2,1,2}-\frac{27}{8}\left(z^{2}-2 z+2\right) H_{2,1,1,0} \\
& +\frac{97}{24}\left(z^{2}-2 z+2\right) H_{1,3,1}-\frac{17}{4}\left(z^{2}-2 z+2\right) H_{1,1,1,0,0}+\frac{289}{60}\left(z^{2}-2 z+2\right) \zeta_{5}
\end{aligned}
$$




$$
\begin{aligned}
& -5\left(z^{2}-2 z+2\right) H_{1,2,2}+\frac{125}{24}\left(z^{2}-2 z+2\right) H_{1,3,0}-\frac{21}{4}\left(z^{2}-2 z+2\right) H_{1,0,0,0,0} \\
& -\frac{21}{4}\left(z^{2}-2 z+2\right) H_{1,1,2,0}+\frac{47}{8}\left(z^{2}-2 z+2\right) H_{3,1,1}+\frac{73}{12}\left(z^{2}-2 z+2\right) H_{1,1,0,0,0} \\
& -\frac{149}{24}\left(z^{2}-2 z+2\right) H_{1,2,1,0}-\frac{269}{24}\left(z^{2}-2 z+2\right) H_{2,1,1,1}-\frac{383}{24}\left(z^{2}-2 z+2\right) H_{1,1,2,1} \\
& -\frac{131}{8}\left(z^{2}-2 z+2\right) H_{1,1,1,2}-\frac{75}{4}\left(z^{2}-2 z+2\right) H_{1,1,1,1,0}-\frac{455}{24}\left(z^{2}-2 z+2\right) H_{1,2,1,1} \\
& -\frac{557}{4}\left(z^{2}-2 z+2\right) H_{1,1,1,1,1}+\frac{1}{48}\left(60 z^{2}-308 z+157\right) H_{1,0,0,0} \\
& +\frac{1}{48}\left(165 z^{2}-218 z+90\right) H_{2,0,0}+\frac{1}{48}\left(235 z^{2}-262 z+90\right) H_{4} \\
& +\frac{1}{48}\left(491 z^{2}-602 z+150\right) H_{3,1}+\frac{1}{48}\left(540 z^{2}-1151 z+234\right) H_{0,0,0} \\
& +\frac{1}{48}\left(595 z^{2}-754 z+270\right) H_{3,0}+\frac{65720 z^{2}-166888 z+102169}{1728} \zeta_{3} \\
& +\frac{81918 z^{2}-60080 z+6689}{1536} \zeta_{4}+\frac{1}{576}\left(18 z^{3}-1349 z^{2}-1405 z+3840\right) H_{0} \\
& +\frac{972 z^{3}-1261663 z^{2}+3374378 z-1847717}{31104} H_{1} \\
& +\frac{-162 z^{4}+108 z^{3}-352897 z^{2}+934550 z-546047}{5184} H_{1,1} \\
& +\frac{1}{288}\left(-9 z^{4}+6 z^{3}-4272 z^{2}+7231 z+564\right) H_{2} \\
& +\frac{1}{288}\left(-9 z^{4}+6 z^{3}-4134 z^{2}+8815 z-1692\right) H_{0,0} \\
& +\frac{1}{192}\left(-6 z^{4}+4 z^{3}-2201 z^{2}+4854 z-2519\right) H_{1,0} \\
& +\frac{1944 z^{4}-1296 z^{3}+1847882 z^{2}-4492864 z+2451781}{20736} \zeta_{2} \\
& +\frac{1}{48}\left(-1109 z^{2}+1906 z-429\right) \zeta_{2} H_{0}+\frac{1}{96}\left(-311 z^{2}+566 z-450\right) \zeta_{2} H_{2} \\
& +\frac{1}{96}\left(-193 z^{2}+990 z-496\right) \zeta_{2} H_{1,0}+\frac{5}{8}\left(z^{2}-2 z+2\right) \zeta_{3} H_{0,0} \\
& +\frac{121}{96}\left(z^{2}-2 z+2\right) \zeta_{4} H_{1}-\frac{21}{16}\left(z^{2}-2 z+2\right) \zeta_{2} H_{2,1}-\frac{67}{48}\left(z^{2}-2 z+2\right) \zeta_{2} H_{1,2} \\
& +\frac{37}{24}\left(z^{2}-2 z+2\right) \zeta_{3} H_{2}+\frac{39}{16}\left(z^{2}-2 z+2\right) \zeta_{2} H_{2,0}+\frac{67}{24}\left(z^{2}-2 z+2\right) \zeta_{3} H_{1,0} \\
& +\frac{45}{16}\left(z^{2}-2 z+2\right) \zeta_{2} H_{3}-\frac{99}{16}\left(z^{2}-2 z+2\right) \zeta_{2} H_{0,0,0}-\frac{135}{16}\left(z^{2}-2 z+2\right) \zeta_{2} H_{1,1,0} \\
& -\frac{1205}{128}\left(z^{2}-2 z+2\right) \zeta_{4} H_{0}+\frac{201}{16}\left(z^{2}-2 z+2\right) \zeta_{2} H_{1,0,0}-\frac{1171}{48}\left(z^{2}-2 z+2\right) \zeta_{2} \zeta_{3} \\
& +\frac{165}{4}\left(z^{2}-2 z+2\right) \zeta_{3} H_{1,1}+\frac{1481}{24}\left(z^{2}-2 z+2\right) \zeta_{2} H_{1,1,1} \\
& +\frac{1}{48}\left(287 z^{2}-166 z-50\right) \zeta_{3} H_{0}+\frac{1}{36}\left(1639 z^{2}-3977 z+2597\right) \zeta_{3} H_{1} \\
& +\frac{1}{96}\left(2527 z^{2}-3030 z+990\right) \zeta_{2} H_{0,0}+\frac{1}{288}\left(18991 z^{2}-46958 z+31295\right) \zeta_{2} H_{1,1} \\
& +\frac{1}{864}\left(59993 z^{2}-152392 z+96547\right) \zeta_{2} H_{1} \\
& H_{g q}^{(0)}=-\frac{17}{12}\left(z^{2}-2 z+2\right) \zeta_{2} H_{1,0}+\frac{97}{36}\left(z^{2}-2 z+2\right) \zeta_{2} H_{1,1}
\end{aligned}
$$




$$
\begin{aligned}
& +\frac{-30797 z^{2}+72940 z-49747}{1296} H_{1,1}+\frac{1}{144}\left(-171 z^{2}+76 z+108\right) H_{0,0} \\
& +\frac{1}{72}\left(-29 z^{2}+82 z-82\right) H_{1,0,0}+\frac{1}{72}\left(-29 z^{2}+82 z-82\right) H_{1,2} \\
& +\frac{1}{24}\left(-8 z^{2}+19 z-17\right) H_{1,0}+\frac{1}{4}\left(-z^{2}+2 z-2\right) H_{1,2,0}+\frac{1}{6}\left(z^{2}-2 z+2\right) H_{1,3} \\
& -\frac{5}{12}\left(z^{2}-2 z+2\right) H_{1,1,0,0}-\frac{5}{12}\left(z^{2}-2 z+2\right) H_{1,1,2}+\frac{5}{6}\left(z^{2}-2 z+2\right) H_{1,0,0,0} \\
& -\frac{11}{12}\left(z^{2}-2 z+2\right) H_{1,2,1}-\frac{80}{9}\left(z^{2}-2 z+2\right) H_{1,1,1,1} \\
& -\frac{2}{27}\left(239 z^{2}-538 z+403\right) H_{1,1,1}-\frac{1}{8} z(3 z-2) H_{2,0}+\frac{5}{12} z(3 z-2) H_{0,0,0} \\
& -\frac{11}{24} z(3 z-2) H_{2,1}+\frac{23}{36}\left(z^{2}-2 z+2\right) \zeta_{3} H_{1}+\left(z^{2}-2 z+2\right) \zeta_{3} H_{0} \\
& +\frac{1}{432}\left(2105 z^{2}-4846 z+3739\right) \zeta_{2} H_{1}+\frac{-104701 z^{2}+254657 z-160076}{3888} H_{1} \\
& +\frac{1}{144}\left(-195 z^{2}+172 z-36\right) H_{2}+\frac{1}{144}\left(-139 z^{2}+412 z-474\right) H_{0} \\
& -\frac{17}{24} z(3 z-2) \zeta_{2} H_{0}+\frac{1}{12} z(3 z-2) H_{3}-\frac{2863}{768}\left(z^{2}-2 z+2\right) \zeta_{4} \\
& +\frac{1}{864}\left(851 z^{2}-898 z-425\right) \zeta_{3}+\frac{42256 z^{2}-84272 z+45221}{5184} \zeta_{2} \\
& +\frac{-2618386 z^{2}+6335144 z-3609251}{93312}, \\
& I_{g q}^{(0)}=\frac{110338 z^{2}-269872 z+165275}{1536}+\frac{1}{32}(27-46 z) H_{1,0,0}+\frac{1}{16}(7-4 z) H_{1,3} \\
& -\frac{3}{16} z(3 z-2) H_{3,1}+\frac{3}{8} z(3 z-2) H_{0,0,0,0}-\frac{3}{8} z(3 z-2) H_{4}-\frac{7}{16} z(3 z-2) H_{3,0} \\
& +\frac{11}{16} z(3 z-2) H_{2,2}+\frac{43}{16} z(3 z-2) H_{2,1,1}+\frac{1}{16} z(3 z+2) H_{2,0,0}+\frac{1}{8} z(6 z-7) H_{3} \\
& +\frac{3}{16} z(17 z-10) H_{2,1,0}+\frac{1}{16}(1-z)(38 z-23) H_{1,1,0}+\frac{1}{32} z(61 z-56) H_{2,0} \\
& +\frac{1}{16} z(67 z-61) H_{2,1}-\frac{1}{32} z(81 z-70) H_{0,0,0}+\frac{-3873 z^{2}-5698 z+5041}{1536} \zeta_{4} \\
& +\frac{1}{192}\left(-3705 z^{2}+8122 z-5584\right) \zeta_{3}+\frac{1}{32}\left(-11 z^{2}-36 z+28\right) H_{1,2} \\
& +\frac{1}{4}\left(z^{2}-2 z+2\right) H_{1,1,3}-\frac{3}{8}\left(z^{2}-2 z+2\right) H_{1,3,1}+\frac{3}{4}\left(z^{2}-2 z+2\right) H_{1,0,0,0,0} \\
& -\frac{3}{4}\left(z^{2}-2 z+2\right) H_{1,4}-\frac{7}{8}\left(z^{2}-2 z+2\right) H_{1,3,0}-\frac{9}{8}\left(z^{2}-2 z+2\right) H_{1,1,0,0,0} \\
& +\frac{9}{8}\left(z^{2}-2 z+2\right) H_{1,1,2,0}+\frac{5}{4}\left(z^{2}-2 z+2\right) H_{1,1,1,0,0}+\frac{5}{4}\left(z^{2}-2 z+2\right) H_{1,1,1,2} \\
& +\frac{11}{8}\left(z^{2}-2 z+2\right) H_{1,2,2}+2\left(z^{2}-2 z+2\right) H_{1,2,1,0}+\frac{5}{2}\left(z^{2}-2 z+2\right) H_{1,1,2,1} \\
& +\frac{117}{40}\left(z^{2}-2 z+2\right) \zeta_{5}+\frac{43}{8}\left(z^{2}-2 z+2\right) H_{1,2,1,1}+\frac{64}{3}\left(z^{2}-2 z+2\right) H_{1,1,1,1,1} \\
& +\frac{3}{8}\left(2 z^{2}-6 z+5\right) H_{1,2,0}-\frac{1}{192} z\left(5 z^{2}+213 z-188\right) H_{0} \\
& -\frac{3}{16}\left(7 z^{2}-18 z+14\right) H_{1,0,0,0}+\frac{3}{16}\left(7 z^{2}-18 z+14\right) H_{1,1,0,0} \\
& +\frac{3}{16}\left(7 z^{2}-18 z+14\right) H_{1,1,2}+\frac{8}{3}\left(15 z^{2}-34 z+25\right) H_{1,1,1,1}
\end{aligned}
$$




$$
\begin{aligned}
& +\frac{1}{16}\left(33 z^{2}-94 z+79\right) H_{1,2,1}+\frac{1}{48}\left(2312 z^{2}-5631 z+3975\right) H_{1,1,1} \\
& +\frac{1}{192}\left(-5 z^{3}+12437 z^{2}-30529 z+19248\right) H_{1}+\frac{1}{192} z\left(5 z^{3}+20 z^{2}+237 z-216\right) H_{2} \\
& +\frac{1}{192} z\left(5 z^{3}+20 z^{2}+399 z-378\right) H_{0,0}-\frac{1}{192}(1-z)\left(5 z^{3}+25 z^{2}-446 z+446\right) H_{1,0} \\
& +\frac{1}{768}\left(-60 z^{4}-240 z^{3}-14708 z^{2}+29916 z-16213\right) \zeta_{2} \\
& +\frac{1}{192}\left(5 z^{4}+20 z^{3}+10840 z^{2}-26620 z+17523\right) H_{1,1}-\frac{29}{32} z(3 z-2) \zeta_{2} H_{0,0} \\
& -\frac{1}{4} z(6 z-5) \zeta_{3} H_{0}-\frac{1}{32} z(63 z-58) \zeta_{2} H_{2}+\frac{1}{32} z(164 z-151) \zeta_{2} H_{0} \\
& +\frac{1}{192}\left(-2333 z^{2}+5680 z-3739\right) \zeta_{2} H_{1}+\frac{1}{24}\left(-321 z^{2}+728 z-539\right) \zeta_{3} H_{1} \\
& +\frac{25}{96}\left(z^{2}-2 z+2\right) \zeta_{2} \zeta_{3}-\frac{9}{8}\left(z^{2}-2 z+2\right) \zeta_{3} H_{1,0}+\frac{229}{192}\left(z^{2}-2 z+2\right) \zeta_{4} H_{1} \\
& -\frac{25}{16}\left(z^{2}-2 z+2\right) \zeta_{2} H_{1,2}-\frac{29}{16}\left(z^{2}-2 z+2\right) \zeta_{2} H_{1,0,0}+\frac{15}{8}\left(z^{2}-2 z+2\right) \zeta_{2} H_{1,1,0} \\
& -\frac{83}{12}\left(z^{2}-2 z+2\right) \zeta_{3} H_{1,1}-\frac{29}{4}\left(z^{2}-2 z+2\right) \zeta_{2} H_{1,1,1}+\frac{3}{4}\left(4 z^{2}-9 z+6\right) \zeta_{2} H_{1,0} \\
& -\frac{5}{16}\left(37 z^{2}-86 z+62\right) \zeta_{2} H_{1,1} .
\end{aligned}
$$

\section{C.3 The $q \bar{q}$ initial state}

The contribution of the two-loop amplitude for $q \bar{q} \rightarrow H g$ to the inclusive Higgs cross section at $\mathrm{N}^{3} \mathrm{LO}$ can be written as

$$
\hat{\sigma}_{q \bar{q} \rightarrow H g}^{(3)}=2\left(\frac{\alpha_{0}}{2 \pi}\right)^{3} s^{-1-3 \epsilon} \sigma_{0} \hat{\sigma}_{q \bar{q} \rightarrow H g}^{(3)} .
$$

The result is regular in the limit $z \rightarrow 1$, and so we do not need to separate off the contribution from the soft limit. The coefficient $\hat{\sigma}_{q \bar{q} \rightarrow H g}^{(3)}$ can be written as

$$
\begin{aligned}
\hat{\sigma}_{q \bar{q} \rightarrow H g}^{(3)}=\sum_{k=-4}^{0} \epsilon^{k} \frac{V}{N}[ & N^{3} A_{q \bar{q}}^{(k)}+N^{2} N_{f} B_{q \bar{q}}^{(k)}+N N_{f}^{2} C_{q \bar{q}}^{(k)}+N_{f} D_{q \bar{q}}^{(k)}+N E_{q \bar{q}}^{(k)} \\
& \left.+\frac{N_{f}^{2}}{N} F_{q \bar{q}}^{(k)}+\frac{1}{N} G_{q \bar{q}}^{(k)}+\frac{N_{f}}{N^{2}} H_{q \bar{q}}^{(k)}+\frac{1}{N^{3}} I_{q \bar{q}}^{(k)}\right]
\end{aligned}
$$

with

$$
\begin{aligned}
& A_{q \bar{q}}^{(-4)}=\frac{1}{6}(1-z)^{3}, \\
& B_{q \bar{q}}^{(-4)}=0, \\
& C_{q \bar{q}}^{(-4)}=0, \\
& D_{q \bar{q}}^{(-4)}=0, \\
& E_{q \bar{q}}^{(-4)}=-\frac{1}{3}(1-z)^{3}, \\
& F_{q \bar{q}}^{(-4)}=0,
\end{aligned}
$$




$$
\begin{aligned}
G_{q \bar{q}}^{(-4)}= & \frac{5}{24}(1-z)^{3}, \\
H_{q \bar{q}}^{(-4)}= & 0 \\
I_{q \bar{q}}^{(-4)}= & -\frac{1}{24}(1-z)^{3}, \\
A_{q \bar{q}}^{(-3)}= & \frac{2}{3}(1-z)^{3} H_{1}-\frac{1}{24}(1-z)^{3}, \\
B_{q \bar{q}}^{(-3)}= & \frac{5}{36}(1-z)^{3}, \\
C_{q \bar{q}}^{(-3)}= & 0, \\
D_{q \bar{q}}^{(-3)}= & -\frac{5}{24}(1-z)^{3}, \\
E_{q \bar{q}}^{(-3)}= & -\frac{7}{6}(1-z)^{3} H_{1}-\frac{35}{144}(1-z)^{3}, \\
F_{q \bar{q}}^{(-3)}= & 0, \\
G_{q \bar{q}}^{(-3)}= & \frac{7}{12}(1-z)^{3} H_{1}+\frac{7}{16}(1-z)^{3}, \\
H_{q \bar{q}}^{(-3)}= & \frac{5}{72}(1-z)^{3}, \\
I_{q \bar{q}}^{(-3)}= & -\frac{1}{12}(1-z)^{3} H_{1}-\frac{11}{72}(1-z)^{3}, \\
H_{q \bar{q}}^{(-2)}= & \frac{5}{36}(1-z)^{3} H_{1}+\frac{31}{108}(1-z)^{3}, \\
& -\frac{1}{3} z\left(2 z^{2}-3 z+3\right) H_{2}+\frac{1}{72}(1-z)\left(89 z^{2}-226 z+89\right) H_{1}-\frac{2}{3}(1-z) z H_{0} \\
F_{q \bar{q}}^{(-2)}= & -\frac{1}{27}(1-z)^{3}, \\
G_{q \bar{q}}^{(-2)}= & -\frac{1}{3} z\left(2 z^{2}-3 z+3\right) H_{0,0}+\frac{1}{2}(1-z)^{3} H_{1,0}+\frac{13}{6}(1-z)^{3} H_{1,1} \\
A_{q \bar{q}}^{(-2)}= & -\frac{1}{6} z\left(2 z^{2}-3 z+3\right) H_{0,0}+\frac{8}{3}(1-z)^{3} H_{1,1}-\frac{1}{6}(1-z)\left(z^{2}+1\right) H_{1} \\
& -\frac{1}{6} z\left(2 z^{2}-3 z+3\right) H_{2}-\frac{1}{3}(1-z) z H_{0}+\frac{1}{288}(1-z)\left(25 z^{2}-86 z-35\right) \\
& +\frac{1}{12}\left(13 z^{3}-33 z^{2}+33 z-9\right) \zeta_{2}, \\
B_{q \bar{q}}^{(-2)}= & \frac{4}{9}(1-z)^{3} H_{1}+\frac{2}{9}(1-z)^{3}, \\
C_{q \bar{q}}^{(-2)}= & \frac{1}{27}(1-z)^{3}, \\
D_{q \bar{q}}^{(-2)}= & -\frac{7}{12}(1-z)^{3} H_{1}-\frac{55}{108}(1-z)^{3}, \\
E_{q \bar{q}}^{(-2)}= & \frac{5}{12} z\left(2 z^{2}-3 z+3\right) H_{0,0}-\frac{1}{3}(1-z)^{3} H_{1,0}-\frac{9}{2}(1-z)^{3} H_{1,1} \\
& \\
&
\end{aligned}
$$




$$
\begin{aligned}
& I_{q \bar{q}}^{(-2)}=\frac{1}{12} z\left(2 z^{2}-3 z+3\right) H_{0,0}-\frac{1}{6}(1-z)^{3} H_{1,0}-\frac{1}{3}(1-z)^{3} H_{1,1} \\
& +\frac{1}{12} z\left(2 z^{2}-3 z+3\right) H_{2}-\frac{1}{36}(1-z)\left(11 z^{2}-28 z+11\right) H_{1}+\frac{1}{6}(1-z) z H_{0} \\
& -\frac{1}{864}(1-z)\left(493 z^{2}-1148 z+511\right)+\frac{1}{48}\left(-53 z^{3}+147 z^{2}-147 z+45\right) \zeta_{2}, \\
& A_{q \bar{q}}^{(-1)}=-\frac{1}{2} z\left(2 z^{2}-3 z+3\right) H_{2,0}+\frac{7}{12} z\left(2 z^{2}-3 z+3\right) H_{0,0,0} \\
& -\frac{7}{6} z\left(2 z^{2}-3 z+3\right) H_{2,1}-\frac{1}{3}(1-z)\left(2 z^{2}+3 z+2\right) H_{1,1} \\
& +\frac{1}{72} z\left(28 z^{2}-81 z+27\right) H_{0,0}+\frac{1}{6}(1-z)^{3} H_{1,0,0}+\frac{1}{6}(1-z)^{3} H_{1,2} \\
& +\frac{32}{3}(1-z)^{3} H_{1,1,1}-((1-z) z) H_{1,0}-\frac{2}{3} z\left(2 z^{2}-3 z+3\right) \zeta_{2} H_{0} \\
& -\frac{1}{12} z\left(2 z^{2}-3 z+3\right) H_{3}+\frac{1}{72} z\left(28 z^{2}+15 z-69\right) H_{2} \\
& +\frac{1}{432}(1-z)\left(35 z^{2}-802 z-397\right) H_{1}-\frac{19}{6}(1-z)^{3} \zeta_{2} H_{1}+\frac{1}{36}(1-z) z(6 z-49) H_{0} \\
& +\frac{(1-z)\left(9481 z^{2}-28196 z+4747\right)}{5184}+\frac{1}{144}\left(-127 z^{3}+399 z^{2}-291 z+71\right) \zeta_{2} \\
& +\frac{1}{12}\left(38 z^{3}-105 z^{2}+105 z-32\right) \zeta_{3}, \\
& B_{q \bar{q}}^{(-1)}=-\frac{1}{9} z\left(2 z^{2}-3 z+3\right) H_{0,0}+\frac{13}{9}(1-z)^{3} H_{1,1}-\frac{1}{9} z\left(2 z^{2}-3 z+3\right) H_{2} \\
& -\frac{2}{9}(1-z) z H_{0}+\frac{1}{18}(1-z)(3 z-5)(5 z-3) H_{1}+\frac{1}{432}(1-z)\left(19 z^{2}-74 z-41\right) \\
& +\frac{1}{72}\left(77 z^{3}-207 z^{2}+207 z-61\right) \zeta_{2} \\
& C_{q \bar{q}}^{(-1)}=\frac{2}{27}(1-z)^{3} H_{1}+\frac{4}{27}(1-z)^{3}, \\
& D_{q \bar{q}}^{(-1)}=\frac{2}{9} z\left(2 z^{2}-3 z+3\right) H_{0,0}-\frac{2}{9}(1-z)^{3} H_{1,0}-\frac{35}{18}(1-z)^{3} H_{1,1} \\
& +\frac{2}{9} z\left(2 z^{2}-3 z+3\right) H_{2}-\frac{2}{27}(1-z)\left(19 z^{2}-44 z+19\right) H_{1}+\frac{4}{9}(1-z) z H_{0} \\
& -\frac{1}{36}(1-z)\left(37 z^{2}-86 z+33\right)+\frac{1}{144}\left(-391 z^{3}+1077 z^{2}-1077 z+327\right) \zeta_{2}, \\
& E_{q \bar{q}}^{(-1)}=-\frac{1}{3}(1-z)\left(z^{2}-9 z+1\right) H_{1,0}+\frac{7}{6} z\left(2 z^{2}-3 z+3\right) H_{2,0} \\
& -\frac{17}{12} z\left(2 z^{2}-3 z+3\right) H_{0,0,0}+\frac{11}{4} z\left(2 z^{2}-3 z+3\right) H_{2,1} \\
& +\frac{1}{72} z\left(22 z^{2}+27 z+45\right) H_{0,0}-\frac{1}{18}(1-z)\left(47 z^{2}-193 z+47\right) H_{1,1} \\
& -\frac{2}{3}(1-z)^{3} H_{1,0,0}-2(1-z)^{3} H_{1,2}-\frac{8}{3}(1-z)^{3} H_{1,1,0}-\frac{115}{6}(1-z)^{3} H_{1,1,1} \\
& +\frac{13}{6} z\left(2 z^{2}-3 z+3\right) \zeta_{2} H_{0}+\frac{1}{6} z\left(2 z^{2}-3 z+3\right) H_{3}+\frac{1}{72} z\left(22 z^{2}-201 z+273\right) H_{2} \\
& -\frac{1}{432}(1-z)\left(1123 z^{2}-4655 z+592\right) H_{1}+10(1-z)^{3} \zeta_{2} H_{1} \\
& -\frac{1}{36}(1-z) z(9 z-131) H_{0}-\frac{(1-z)\left(15149 z^{2}-55138 z+4709\right)}{5184} \\
& +\frac{1}{288}\left(-459 z^{3}+573 z^{2}-861 z+371\right) \zeta_{2}+\frac{1}{24}\left(-155 z^{3}+423 z^{2}-423 z+127\right) \zeta_{3},
\end{aligned}
$$




$$
\begin{aligned}
& F_{q \bar{q}}^{(-1)}=-\frac{2}{27}(1-z)^{3} H_{1}-\frac{4}{27}(1-z)^{3}, \\
& G_{q \bar{q}}^{(-1)}=-\frac{5}{6} z\left(2 z^{2}-3 z+3\right) H_{2,0}+\frac{13}{12} z\left(2 z^{2}-3 z+3\right) H_{0,0,0} \\
& -2 z\left(2 z^{2}-3 z+3\right) H_{2,1}+\frac{1}{18}(1-z)\left(19 z^{2}-68 z+19\right) H_{1,0} \\
& -\frac{1}{24} z\left(34 z^{2}-43 z+49\right) H_{0,0}+\frac{1}{18}(1-z)\left(83 z^{2}-238 z+83\right) H_{1,1} \\
& +\frac{2}{3}(1-z)^{3} H_{1,0,0}+\frac{5}{2}(1-z)^{3} H_{1,2}+\frac{11}{3}(1-z)^{3} H_{1,1,0}+\frac{31}{3}(1-z)^{3} H_{1,1,1} \\
& -\frac{7}{3} z\left(2 z^{2}-3 z+3\right) \zeta_{2} H_{0}-\frac{1}{12} z\left(2 z^{2}-3 z+3\right) H_{3}-\frac{1}{24} z\left(34 z^{2}-99 z+105\right) H_{2} \\
& +\frac{1}{144}(1-z)\left(527 z^{2}-1846 z+503\right) H_{1}-\frac{67}{8}(1-z)^{3} \zeta_{2} H_{1} \\
& +\frac{1}{12}(1-z)(z-40) z H_{0}+\frac{(1-z)\left(14687 z^{2}-52756 z+8693\right)}{5184} \\
& +\frac{1}{12}\left(52 z^{3}-141 z^{2}+141 z-42\right) \zeta_{3}+\frac{1}{288}\left(1939 z^{3}-4389 z^{2}+4461 z-1531\right) \zeta_{2}, \\
& H_{q \bar{q}}^{(-1)}=-\frac{1}{9} z\left(2 z^{2}-3 z+3\right) H_{0,0}+\frac{2}{9}(1-z)^{3} H_{1,0}+\frac{1}{2}(1-z)^{3} H_{1,1} \\
& -\frac{1}{9} z\left(2 z^{2}-3 z+3\right) H_{2}+\frac{1}{54}(1-z)\left(31 z^{2}-74 z+31\right) H_{1}-\frac{2}{9}(1-z) z H_{0} \\
& +\frac{1}{432}(1-z)\left(425 z^{2}-958 z+437\right)+\frac{1}{144}\left(237 z^{3}-663 z^{2}+663 z-205\right) \zeta_{2}, \\
& I_{q \bar{q}}^{(-1)}=\frac{1}{6} z\left(2 z^{2}-3 z+3\right) H_{2,0}-\frac{1}{4} z\left(2 z^{2}-3 z+3\right) H_{0,0,0} \\
& +\frac{5}{12} z\left(2 z^{2}-3 z+3\right) H_{2,1}-\frac{1}{6}(1-z)\left(8 z^{2}-21 z+8\right) H_{1,1} \\
& -\frac{1}{18}(1-z)\left(13 z^{2}-32 z+13\right) H_{1,0}+\frac{1}{72} z\left(52 z^{2}-75 z+75\right) H_{0,0} \\
& -\frac{1}{6}(1-z)^{3} H_{1,0,0}-\frac{2}{3}(1-z)^{3} H_{1,2}-(1-z)^{3} H_{1,1,0}-\frac{11}{6}(1-z)^{3} H_{1,1,1} \\
& +\frac{5}{6} z\left(2 z^{2}-3 z+3\right) \zeta_{2} H_{0}+\frac{1}{72} z\left(52 z^{2}-111 z+111\right) H_{2} \\
& -\frac{1}{432}(1-z)\left(493 z^{2}-1685 z+520\right) H_{1}+\frac{37}{24}(1-z)^{3} \zeta_{2} H_{1}+\frac{19}{18}(1-z) z H_{0} \\
& -\frac{(1-z)\left(9019 z^{2}-25814 z+8731\right)}{5184}+\frac{1}{144}\left(-613 z^{3}+1509 z^{2}-1509 z+509\right) \zeta_{2} \\
& +\frac{1}{24}\left(-25 z^{3}+69 z^{2}-69 z+21\right) \zeta_{3},
\end{aligned}
$$

$$
\begin{aligned}
A_{q \bar{q}}^{(0)}= & \frac{(1-z)\left(104741 z^{2}-281554 z+36581\right)}{10368}-\frac{1}{6}(1-z)^{3} H_{1,3} \\
& +\frac{1}{6}(1-z)^{3} H_{1,1,0,0}+\frac{1}{3}(1-z)^{3} H_{1,2,1}+\frac{1}{3}(1-z)^{3} H_{1,1,2}-\frac{1}{2}(1-z)^{3} H_{1,0,0,0} \\
& -\frac{2}{3}(1-z)^{3} H_{1,1,1,0}+\frac{253}{6}(1-z)^{3} H_{1,1,1,1}-\frac{1}{18}(1-z)^{2}(13 z-94) H_{1,2} \\
& -\frac{1}{864}(1-z) z(765 z-2927) H_{0}+\frac{1}{8} z\left(2 z^{2}-14 z-3\right) H_{2,0} \\
& +\frac{5}{12} z\left(2 z^{2}-3 z+3\right) H_{2,0,0}+\frac{3}{4} z\left(2 z^{2}-3 z+3\right) H_{4}-\frac{3}{2} z\left(2 z^{2}-3 z+3\right) H_{2,2}
\end{aligned}
$$




$$
\begin{aligned}
& +\frac{19}{12} z\left(2 z^{2}-3 z+3\right) H_{3,1}-\frac{19}{12} z\left(2 z^{2}-3 z+3\right) H_{2,1,0}-\frac{7}{4} z\left(2 z^{2}-3 z+3\right) H_{0,0,0,0} \\
& +\frac{7}{4} z\left(2 z^{2}-3 z+3\right) H_{3,0}-\frac{37}{6} z\left(2 z^{2}-3 z+3\right) H_{2,1,1} \\
& -\frac{1}{12}(1-z)\left(11 z^{2}+16 z+11\right) H_{1,1,0}+\frac{1}{18}(1-z)\left(13 z^{2}-38 z+94\right) H_{1,0,0} \\
& -\frac{1}{12} z\left(16 z^{2}-11 z+17\right) H_{3}-\frac{1}{12}(1-z)\left(43 z^{2}+62 z+43\right) H_{1,1,1} \\
& +\frac{1}{24} z\left(58 z^{2}-47 z-85\right) H_{2,1}-\frac{1}{432}(1-z)\left(61 z^{2}+1507 z+394\right) H_{1,0} \\
& -\frac{1}{24} z\left(84 z^{2}-155 z+86\right) H_{0,0,0}-\frac{1}{216}(1-z)\left(107 z^{2}+2027 z+1196\right) H_{1,1} \\
& +\frac{1}{432} z\left(340 z^{2}+2694 z-2217\right) H_{2}+\frac{1}{432} z\left(745 z^{2}-6 z+78\right) H_{0,0} \\
& +\frac{(1-z)\left(4885 z^{2}-13745 z-8669\right)}{1296} H_{1}+\frac{-585 z^{3}-4785 z^{2}-615 z+2717}{1728} \zeta_{2} \\
& +\frac{1}{72}\left(-167 z^{3}+378 z^{2}-576 z+449\right) \zeta_{3}+\frac{1}{96}\left(1447 z^{3}-1929 z^{2}+1929 z+161\right) \zeta_{4} \\
& +3(1-z)^{3} \zeta_{2} H_{1,0}-\frac{65}{6}(1-z)^{3} \zeta_{3} H_{1}-\frac{71}{6}(1-z)^{3} \zeta_{2} H_{1,1} \\
& -\frac{1}{12} z\left(2 z^{2}-3 z+3\right) \zeta_{2} H_{2}+\frac{2}{3} z\left(2 z^{2}-3 z+3\right) \zeta_{3} H_{0}+\frac{13}{6} z\left(2 z^{2}-3 z+3\right) \zeta_{2} H_{0,0} \\
& +\frac{1}{18}(1-z)\left(50 z^{2}-76 z-31\right) \zeta_{2} H_{1}+\frac{1}{24} z\left(74 z^{2}-186 z+153\right) \zeta_{2} H_{0}, \\
& B_{q \bar{q}}^{(0)}=-\frac{1}{6} z\left(2 z^{2}-3 z+3\right) H_{2,0}+\frac{5}{9} z\left(2 z^{2}-3 z+3\right) H_{0,0,0} \\
& -\frac{11}{18} z\left(2 z^{2}-3 z+3\right) H_{2,1}-\frac{1}{18}(1-z)\left(4 z^{2}-17 z+31\right) H_{1,0,0} \\
& -\frac{1}{18}(1-z)\left(4 z^{2}-17 z+31\right) H_{1,2}+\frac{1}{27}(1-z)\left(80 z^{2}-193 z+80\right) H_{1,1} \\
& -\frac{1}{216} z\left(112 z^{2}+177 z-96\right) H_{0,0}+\frac{43}{9}(1-z)^{3} H_{1,1,1}-\frac{1}{3}(1-z) z H_{1,0} \\
& -\frac{17}{18} z\left(2 z^{2}-3 z+3\right) \zeta_{2} H_{0}-\frac{1}{18}(1-z)\left(46 z^{2}-83 z+19\right) \zeta_{2} H_{1} \\
& +\frac{1}{9} z\left(2 z^{2}-3 z+3\right) H_{3}-\frac{1}{216} z\left(112 z^{2}-15 z+96\right) H_{2} \\
& +\frac{1}{432}(1-z)\left(518 z^{2}-2565 z+1133\right) H_{1}+\frac{1}{216}(1-z) z(126 z-703) H_{0} \\
& -\frac{(1-z)\left(13387 z^{2}-8171 z+11236\right)}{7776}+\frac{1}{12}\left(33 z^{3}-99 z^{2}+117 z-47\right) \zeta_{3} \\
& +\frac{1}{216}\left(202 z^{3}+75 z^{2}+6 z-90\right) \zeta_{2} \\
& C_{q \bar{q}}^{(0)}=\frac{4}{27}(1-z)^{3} H_{1,1}+\frac{8}{27}(1-z)^{3} H_{1}-\frac{29}{54}(1-z)^{3} \zeta_{2}+\frac{38}{81}(1-z)^{3}, \\
& D_{q \bar{q}}^{(0)}=\frac{1}{3} z\left(2 z^{2}-3 z+3\right) H_{2,0}-\frac{10}{9} z\left(2 z^{2}-3 z+3\right) H_{0,0,0} \\
& +\frac{11}{9} z\left(2 z^{2}-3 z+3\right) H_{2,1}+\frac{1}{18}(1-z)\left(4 z^{2}-17 z+31\right) H_{1,0,0} \\
& -\frac{1}{6}(1-z)\left(4 z^{2}-5 z-5\right) H_{1,2}-\frac{2}{27}(1-z)\left(19 z^{2}-47 z+19\right) H_{1,0} \\
& -\frac{1}{27}(1-z)\left(149 z^{2}-364 z+149\right) H_{1,1}+\frac{1}{216} z\left(416 z^{2}-213 z+276\right) H_{0,0}
\end{aligned}
$$




$$
\begin{aligned}
& -\frac{13}{9}(1-z)^{3} H_{1,1,0}-\frac{23}{3}(1-z)^{3} H_{1,1,1}+\frac{17}{9} z\left(2 z^{2}-3 z+3\right) \zeta_{2} H_{0} \\
& +\frac{1}{24}(1-z)\left(143 z^{2}-274 z+107\right) \zeta_{2} H_{1}-\frac{2}{9} z\left(2 z^{2}-3 z+3\right) H_{3} \\
& +\frac{1}{216} z\left(416 z^{2}-597 z+660\right) H_{2}-\frac{1}{432}(1-z)\left(1368 z^{2}-5413 z+2031\right) H_{1} \\
& -\frac{1}{216}(1-z) z(144 z-1061) H_{0}-\frac{(1-z)\left(2198 z^{2}-17401 z+3119\right)}{2592} \\
& +\frac{1}{216}\left(-1981 z^{3}+4572 z^{2}-4635 z+1565\right) \zeta_{2} \\
& +\frac{1}{72}\left(-253 z^{3}+723 z^{2}-831 z+313\right) \zeta_{3}, \\
& E_{q \bar{q}}^{(0)}=-\frac{(1-z)\left(338279 z^{2}-1388872 z-39343\right)}{31104}+\frac{1}{6}(1-z)^{3} H_{1,3} \\
& -\frac{13}{6}(1-z)^{3} H_{1,1,0,0}-\frac{7}{3}(1-z)^{3} H_{1,2,0}+3(1-z)^{3} H_{1,0,0,0}-\frac{21}{2}(1-z)^{3} H_{1,2,1} \\
& -\frac{27}{2}(1-z)^{3} H_{1,1,2}-\frac{103}{6}(1-z)^{3} H_{1,1,1,0}-90(1-z)^{3} H_{1,1,1,1} \\
& +\frac{31}{288}(1-z) z(5 z+31) H_{0}-\frac{4}{3} z\left(2 z^{2}-3 z+3\right) H_{4}-\frac{17}{6} z\left(2 z^{2}-3 z+3\right) H_{3,1} \\
& -\frac{43}{12} z\left(2 z^{2}-3 z+3\right) H_{3,0}+\frac{23}{6} z\left(2 z^{2}-3 z+3\right) H_{2,2}+4 z\left(2 z^{2}-3 z+3\right) H_{0,0,0,0} \\
& +\frac{179}{12} z\left(2 z^{2}-3 z+3\right) H_{2,1,1}-\frac{1}{12} z\left(20 z^{2}-33 z+33\right) H_{2,0,0} \\
& -\frac{1}{12}(1-z)\left(40 z^{2}-183 z+43\right) H_{1,1,0}+\frac{1}{24} z\left(71 z^{2}-130 z+46\right) H_{0,0,0} \\
& +\frac{1}{24} z\left(83 z^{2}-79 z+88\right) H_{3}+\frac{1}{12} z\left(100 z^{2}-147 z+147\right) H_{2,1,0} \\
& -\frac{1}{72}(1-z)\left(193 z^{2}-437 z+562\right) H_{1,0,0}-\frac{1}{18}(1-z)\left(205 z^{2}-947 z+205\right) H_{1,1,1} \\
& +\frac{1}{72} z\left(238 z^{2}-279 z+351\right) H_{2,0}+\frac{1}{72} z\left(274 z^{2}-1002 z+1353\right) H_{2,1} \\
& -\frac{1}{72}(1-z)\left(307 z^{2}-1319 z+658\right) H_{1,2}-\frac{(1-z)\left(23594 z^{2}-101827 z-12595\right)}{2592} H_{1} \\
& +\frac{1}{96}\left(-1193 z^{3}-465 z^{2}+465 z-1395\right) \zeta_{4}+\frac{1}{144}\left(-249 z^{3}+1269 z^{2}-999 z-583\right) \zeta_{3} \\
& +\frac{1}{144} z\left(9 z^{3}-131 z^{2}-388 z+10\right) H_{0,0}+\frac{1}{144} z\left(9 z^{3}+58 z^{2}-2608 z+2041\right) H_{2} \\
& -\frac{1}{432}(1-z)\left(27 z^{3}-74 z^{2}-5357 z+232\right) H_{1,0} \\
& -\frac{1}{432}(1-z)\left(27 z^{3}+3431 z^{2}-21709 z+1613\right) H_{1,1} \\
& +\frac{1}{576}\left(-108 z^{4}-385 z^{3}+6669 z^{2}-4755 z+579\right) \zeta_{2}+\frac{1}{6}(1-z)^{3} \zeta_{2} H_{1,0} \\
& +\frac{103}{4}(1-z)^{3} \zeta_{3} H_{1}+\frac{183}{4}(1-z)^{3} \zeta_{2} H_{1,1}-\frac{239}{24} z\left(2 z^{2}-3 z+3\right) \zeta_{2} H_{0,0} \\
& -\frac{1}{2} z\left(9 z^{2}-14 z+14\right) \zeta_{3} H_{0}-\frac{1}{8} z\left(34 z^{2}-55 z+55\right) \zeta_{2} H_{2} \\
& -\frac{1}{72} z\left(352 z^{2}-1299 z+813\right) \zeta_{2} H_{0}+\frac{1}{144}(1-z)\left(1651 z^{2}-4148 z+2281\right) \zeta_{2} H_{1} \text {, } \\
& F_{q \bar{q}}^{(0)}=-\frac{4}{27}(1-z)^{3} H_{1,1}-\frac{8}{27}(1-z)^{3} H_{1}+\frac{29}{54}(1-z)^{3} \zeta_{2}-\frac{38}{81}(1-z)^{3},
\end{aligned}
$$




$$
\begin{aligned}
& G_{q \bar{q}}^{(0)}=\frac{(1-z)\left(59869 z^{2}-380522 z+22933\right)}{10368}-\frac{1}{2}(1-z)^{3} H_{1,3} \\
& +\frac{5}{2}(1-z)^{3} H_{1,1,0,0}+\frac{8}{3}(1-z)^{3} H_{1,2,0}-\frac{10}{3}(1-z)^{3} H_{1,0,0,0}+\frac{37}{3}(1-z)^{3} H_{1,2,1} \\
& +16(1-z)^{3} H_{1,1,2}+\frac{68}{3}(1-z)^{3} H_{1,1,1,0}+\frac{343}{6}(1-z)^{3} H_{1,1,1,1} \\
& +\frac{3}{4}(1-z)(2 z-5)(4 z-1) H_{1,2}+\frac{1}{144}(1-z) z(41 z-1694) H_{0} \\
& +\frac{7}{12} z\left(2 z^{2}-3 z+3\right) H_{2,0,0}+\frac{3}{4} z\left(2 z^{2}-3 z+3\right) H_{4}+\frac{19}{12} z\left(2 z^{2}-3 z+3\right) H_{3,1} \\
& +\frac{9}{4} z\left(2 z^{2}-3 z+3\right) H_{3,0}-\frac{5}{2} z\left(2 z^{2}-3 z+3\right) H_{2,2}-\frac{11}{4} z\left(2 z^{2}-3 z+3\right) H_{0,0,0,0} \\
& -\frac{37}{12} z\left(2 z^{2}-3 z+3\right) H_{2,1,0}-\frac{31}{3} z\left(2 z^{2}-3 z+3\right) H_{2,1,1}-\frac{7}{36} z\left(10 z^{2}-9 z+9\right) H_{3} \\
& +\frac{1}{36} z\left(91 z^{2}-153 z+189\right) H_{0,0,0}+\frac{1}{36}(1-z)\left(94 z^{2}-128 z+13\right) H_{1,0,0} \\
& +\frac{2}{9}(1-z)\left(98 z^{2}-289 z+98\right) H_{1,1,1}-\frac{1}{24} z\left(220 z^{2}-523 z+511\right) H_{2,1} \\
& +\frac{1}{36}(1-z)\left(289 z^{2}-800 z+289\right) H_{1,1,0}-\frac{1}{72} z\left(338 z^{2}-537 z+429\right) H_{2,0} \\
& +\frac{(1-z)\left(22681 z^{2}-118154 z+21043\right)}{2592} H_{1} \\
& +\frac{1}{384}\left(-289 z^{3}+12531 z^{2}-12531 z+8065\right) \zeta_{4} \\
& -\frac{1}{144} z\left(18 z^{3}+442 z^{2}-655 z+106\right) H_{0,0}-\frac{1}{144} z\left(18 z^{3}+496 z^{2}-2403 z+1800\right) H_{2} \\
& +\frac{1}{216}(1-z)\left(27 z^{3}+494 z^{2}-3379 z+872\right) H_{1,0} \\
& +\frac{1}{216}(1-z)\left(27 z^{3}+2816 z^{2}-12274 z+3095\right) H_{1,1} \\
& +\frac{1}{144}\left(1111 z^{3}-3855 z^{2}+4539 z-1187\right) \zeta_{3} \\
& +\frac{648 z^{4}+26537 z^{3}-69903 z^{2}+68139 z-26489}{1728} \zeta_{2}-\frac{5}{4}(1-z)^{3} \zeta_{2} H_{1,0} \\
& -\frac{37}{2}(1-z)^{3} \zeta_{3} H_{1}-\frac{397}{12}(1-z)^{3} \zeta_{2} H_{1,1}+\frac{5}{6} z\left(2 z^{2}-3 z+3\right) \zeta_{2} H_{2} \\
& +2 z\left(2 z^{2}-3 z+3\right) \zeta_{3} H_{0}+\frac{133}{12} z\left(2 z^{2}-3 z+3\right) \zeta_{2} H_{0,0} \\
& -\frac{1}{72} z\left(542 z^{2}-171 z+423\right) \zeta_{2} H_{0}-\frac{1}{144}(1-z)\left(2449 z^{2}-5066 z+2125\right) \zeta_{2} H_{1}, \\
& H_{q \bar{q}}^{(0)}=-\frac{1}{6} z\left(2 z^{2}-3 z+3\right) H_{2,0}+\frac{5}{9} z\left(2 z^{2}-3 z+3\right) H_{0,0,0} \\
& -\frac{11}{18} z\left(2 z^{2}-3 z+3\right) H_{2,1}+\frac{1}{9}(1-z)\left(23 z^{2}-57 z+23\right) H_{1,1} \\
& +\frac{1}{27}(1-z)\left(38 z^{2}-85 z+38\right) H_{1,0}-\frac{1}{108} z\left(152 z^{2}-195 z+186\right) H_{0,0} \\
& +\frac{8}{9}(1-z)^{3} H_{1,2}+\frac{13}{9}(1-z)^{3} H_{1,1,0}+\frac{26}{9}(1-z)^{3} H_{1,1,1}-\frac{17}{18} z\left(2 z^{2}-3 z+3\right) \zeta_{2} H_{0} \\
& +\frac{1}{9} z\left(2 z^{2}-3 z+3\right) H_{3}-\frac{1}{108} z\left(152 z^{2}-291 z+282\right) H_{2} \\
& +\frac{1}{216}(1-z)\left(425 z^{2}-1424 z+449\right) H_{1}-\frac{245}{72}(1-z)^{3} \zeta_{2} H_{1}
\end{aligned}
$$




$$
\begin{aligned}
& +\frac{1}{108}(1-z) z(9 z-179) H_{0}+\frac{(1-z)\left(19981 z^{2}-60374 z+20593\right)}{7776} \\
& +\frac{1}{72}\left(55 z^{3}-129 z^{2}+129 z-31\right) \zeta_{3}+\frac{1}{216}\left(1779 z^{3}-4647 z^{2}+4629 z-1475\right) \zeta_{2}, \\
& I_{q \bar{q}}^{(0)}=-\frac{(1-z)\left(155551 z^{2}-597356 z+217885\right)}{31104}-\frac{1}{3}(1-z)^{3} H_{1,2,0} \\
& +\frac{1}{2}(1-z)^{3} H_{1,3}-\frac{1}{2}(1-z)^{3} H_{1,1,0,0}+\frac{5}{6}(1-z)^{3} H_{1,0,0,0}-\frac{13}{6}(1-z)^{3} H_{1,2,1} \\
& -\frac{17}{6}(1-z)^{3} H_{1,1,2}-\frac{29}{6}(1-z)^{3} H_{1,1,1,0}-\frac{28}{3}(1-z)^{3} H_{1,1,1,1} \\
& +\frac{1}{432}(1-z) z(27 z+2177) H_{0}-\frac{1}{6} z\left(2 z^{2}-3 z+3\right) H_{4}+\frac{1}{6} z\left(2 z^{2}-3 z+3\right) H_{2,2} \\
& -\frac{1}{3} z\left(2 z^{2}-3 z+3\right) H_{3,1}-\frac{5}{12} z\left(2 z^{2}-3 z+3\right) H_{3,0}+\frac{1}{2} z\left(2 z^{2}-3 z+3\right) H_{0,0,0,0} \\
& +\frac{19}{12} z\left(2 z^{2}-3 z+3\right) H_{2,1,1}+\frac{1}{4} z\left(4 z^{2}-7 z+7\right) H_{2,1,0}-\frac{1}{12} z\left(4 z^{2}-3 z+3\right) H_{2,0,0} \\
& -\frac{1}{72} z\left(13 z^{2}-45 z+36\right) H_{3}-\frac{1}{72}(1-z)\left(47 z^{2}+29 z-160\right) H_{1,0,0} \\
& -\frac{1}{24}(1-z)\left(59 z^{2}-99 z-4\right) H_{1,2}+\frac{1}{72} z\left(82 z^{2}-132 z+105\right) H_{2,0} \\
& -\frac{1}{36}(1-z)\left(136 z^{2}-299 z+127\right) H_{1,1,0}-\frac{1}{72} z\left(143 z^{2}-231 z+258\right) H_{0,0,0} \\
& +\frac{1}{72} z\left(212 z^{2}-426 z+435\right) H_{2,1}-\frac{1}{36}(1-z)\left(245 z^{2}-604 z+245\right) H_{1,1,1} \\
& -\frac{(1-z)\left(8857 z^{2}-43817 z+16300\right)}{2592} H_{1}+\frac{1}{384}\left(-727 z^{3}-2955 z^{2}+2955 z-3129\right) \zeta_{4} \\
& +\frac{1}{72}\left(-264 z^{3}+915 z^{2}-1194 z+436\right) \zeta_{3}+\frac{1}{432} z\left(27 z^{3}+974 z^{2}-2079 z+1494\right) H_{2} \\
& +\frac{1}{432} z\left(27 z^{3}+974 z^{2}-795 z+210\right) H_{0,0} \\
& -\frac{1}{432}(1-z)\left(27 z^{3}+1001 z^{2}-2908 z+1118\right) H_{1,0} \\
& -\frac{1}{432}(1-z)\left(27 z^{3}+1987 z^{2}-6893 z+2185\right) H_{1,1} \\
& +\frac{-324 z^{4}-24797 z^{3}+54681 z^{2}-53259 z+22035}{1728} \zeta_{2}-\frac{5}{6}(1-z)^{3} \zeta_{2} H_{1,1} \\
& -\frac{23}{12}(1-z)^{3} \zeta_{2} H_{1,0}+\frac{43}{12}(1-z)^{3} \zeta_{3} H_{1}-\frac{79}{24} z\left(2 z^{2}-3 z+3\right) \zeta_{2} H_{0,0} \\
& -\frac{1}{6} z\left(5 z^{2}-6 z+6\right) \zeta_{3} H_{0}+\frac{1}{8} z\left(22 z^{2}-37 z+37\right) \zeta_{2} H_{2} \\
& +\frac{1}{72}(1-z)\left(199 z^{2}-155 z+46\right) \zeta_{2} H_{1}+\frac{1}{24} z\left(224 z^{2}-304 z+259\right) \zeta_{2} H_{0} \text {. }
\end{aligned}
$$

Open Access. This article is distributed under the terms of the Creative Commons Attribution License (CC-BY 4.0), which permits any use, distribution and reproduction in any medium, provided the original author(s) and source are credited.

\section{References}

[1] G. Altarelli and G. Parisi, Asymptotic freedom in parton language, Nucl. Phys. B 126 (1977) 298 [inSPIRE]. 
[2] A. Bassetto, M. Ciafaloni and G. Marchesini, Jet structure and infrared sensitive quantities in perturbative QCD, Phys. Rept. 100 (1983) 201 [INSPIRE].

[3] Yu.L. Dokshitser, V.A. Khoze, A.H. Mueller and S.I. Troian, Basics of perturbative QCD, Editions Frontières, Gif-sur-Yvette France (1991) [INSPIRE] and references therein.

[4] S. Frixione, Z. Kunszt and A. Signer, Three jet cross-sections to next-to-leading order, Nucl. Phys. B 467 (1996) 399 [hep-ph/9512328] [INSPIRE].

[5] S. Catani and M.H. Seymour, A general algorithm for calculating jet cross-sections in NLO QCD, Nucl. Phys. B 485 (1997) 291 [Erratum ibid. B 510 (1998) 503] [hep-ph/9605323] [INSPIRE].

[6] D.A. Kosower, Antenna factorization of gauge theory amplitudes, Phys. Rev. D 57 (1998) 5410 [hep-ph/9710213] [INSPIRE].

[7] D.A. Kosower, All order collinear behavior in gauge theories, Nucl. Phys. B 552 (1999) 319 [hep-ph/9901201] [INSPIRE].

[8] S. Catani, The singular behavior of QCD amplitudes at two loop order, Phys. Lett. B 427 (1998) 161 [hep-ph/9802439] [INSPIRE].

[9] G.F. Sterman and M.E. Tejeda-Yeomans, Multiloop amplitudes and resummation, Phys. Lett. B 552 (2003) 48 [hep-ph/0210130] [INSPIRE].

[10] T. Becher and M. Neubert, Infrared singularities of scattering amplitudes in perturbative QCD, Phys. Rev. Lett. 102 (2009) 162001 [Erratum ibid. 111 (2013) 199905] [arXiv: 0901.0722] [INSPIRE].

[11] E. Gardi and L. Magnea, Factorization constraints for soft anomalous dimensions in QCD scattering amplitudes, JHEP 03 (2009) 079 [arXiv:0901.1091] [INSPIRE].

[12] J.M. Campbell and E.W.N. Glover, Double unresolved approximations to multiparton scattering amplitudes, Nucl. Phys. B 527 (1998) 264 [hep-ph/9710255] [INSPIRE].

[13] S. Catani and M. Grazzini, Collinear factorization and splitting functions for next-to-next-to-leading order QCD calculations, Phys. Lett. B 446 (1999) 143 [hep-ph/9810389] [INSPIRE].

[14] S. Catani and M. Grazzini, The soft gluon current at one loop order, Nucl. Phys. B 591 (2000) 435 [hep-ph/0007142] [INSPIRE].

[15] A. Gehrmann-De Ridder, T. Gehrmann and E.W.N. Glover, Antenna subtraction at NNLO, JHEP 09 (2005) 056 [hep-ph/0505111] [INSPIRE].

[16] S. Catani and M. Grazzini, An NNLO subtraction formalism in hadron collisions and its application to Higgs boson production at the LHC, Phys. Rev. Lett. 98 (2007) 222002 [hep-ph/0703012] [INSPIRE].

[17] M. Czakon, Double-real radiation in hadronic top quark pair production as a proof of a certain concept, Nucl. Phys. B 849 (2011) 250 [arXiv:1101.0642] [InSPIRE].

[18] T.G. Birthwright, E.W.N. Glover, V.V. Khoze and P. Marquard, Multi-gluon collinear limits from MHV diagrams, JHEP 05 (2005) 013 [hep-ph/0503063] [INSPIRE].

[19] S. Catani, D. de Florian and G. Rodrigo, The triple collinear limit of one loop QCD amplitudes, Phys. Lett. B 586 (2004) 323 [hep-ph/0312067] [INSPIRE]. 
[20] S. Buchta, G. Chachamis, P. Draggiotis, I. Malamos and G. Rodrigo, On the singular behaviour of scattering amplitudes in quantum field theory, JHEP 11 (2014) 014 [arXiv: 1405.7850] [INSPIRE].

[21] Z. Bern, L.J. Dixon and D.A. Kosower, Two-loop $g \rightarrow$ gg splitting amplitudes in QCD, JHEP 08 (2004) 012 [hep-ph/0404293] [INSPIRE].

[22] S.D. Badger and E.W.N. Glover, Two loop splitting functions in QCD, JHEP 07 (2004) 040 [hep-ph/0405236] [INSPIRE].

[23] L.W. Garland, T. Gehrmann, E.W.N. Glover, A. Koukoutsakis and E. Remiddi, The two loop QCD matrix element for $e^{+} e^{-} \rightarrow 3$ jets, Nucl. Phys. B 627 (2002) 107 [hep-ph/0112081] [INSPIRE].

[24] T. Gehrmann, M. Jaquier, E.W.N. Glover and A. Koukoutsakis, Two-loop QCD corrections to the helicity amplitudes for $H \rightarrow 3$ partons, JHEP 02 (2012) 056 [arXiv:1112.3554] [INSPIRE].

[25] C. Duhr and T. Gehrmann, The two-loop soft current in dimensional regularization, Phys. Lett. B 727 (2013) 452 [arXiv: 1309.4393] [INSPIRE].

[26] Y. Li and H.X. Zhu, Single soft gluon emission at two loops, JHEP 11 (2013) 080 [arXiv:1309.4391] [INSPIRE].

[27] R.J. Gonsalves, Dimensionally regularized two loop on-shell quark form-factor, Phys. Rev. D 28 (1983) 1542 [inSPIRE].

[28] G. Kramer and B. Lampe, Integrals for two loop calculations in massless QCD, J. Math. Phys. 28 (1987) 945 [INSPIRE].

[29] T. Gehrmann, T. Huber and D. Maître, Two-loop quark and gluon form-factors in dimensional regularisation, Phys. Lett. B 622 (2005) 295 [hep-ph/0507061] [INSPIRE].

[30] P.A. Baikov, K.G. Chetyrkin, A.V. Smirnov, V.A. Smirnov and M. Steinhauser, Quark and gluon form factors to three loops, Phys. Rev. Lett. 102 (2009) 212002 [arXiv:0902.3519] [INSPIRE].

[31] T. Gehrmann, E.W.N. Glover, T. Huber, N. Ikizlerli and C. Studerus, Calculation of the quark and gluon form factors to three loops in QCD, JHEP 06 (2010) 094 [arXiv: 1004.3653] [INSPIRE].

[32] M. Höschele, J. Hoff, A. Pak, M. Steinhauser and T. Ueda, Higgs boson production at the LHC: NNLO partonic cross sections through order $\epsilon$ and convolutions with splitting functions to $N^{3} L O$, Phys. Lett. B 721 (2013) 244 [arXiv:1211.6559] [INSPIRE].

[33] S. Buehler and A. Lazopoulos, Scale dependence and collinear subtraction terms for Higgs production in gluon fusion at $N^{3} L O, J H E P 10$ (2013) 096 [arXiv:1306.2223] [INSPIRE].

[34] C. Anastasiou, C. Duhr, F. Dulat and B. Mistlberger, Soft triple-real radiation for Higgs production at $N^{3} L O$, JHEP 07 (2013) 003 [arXiv: 1302.4379] [INSPIRE].

[35] C. Anastasiou, C. Duhr, F. Dulat, F. Herzog and B. Mistlberger, Real-virtual contributions to the inclusive Higgs cross-section at $N^{3} L O$, JHEP 12 (2013) 088 [arXiv:1311.1425] [INSPIRE].

[36] Y. Li, A. von Manteuffel, R.M. Schabinger and H.X. Zhu, $N^{3}$ LO Higgs boson and Drell-Yan production at threshold: the one-loop two-emission contribution, Phys. Rev. D 90 (2014) 053006 [arXiv:1404.5839] [INSPIRE]. 
[37] C. Anastasiou et al., Higgs boson gluon-fusion production at threshold in $N^{3} L O Q C D$, Phys. Lett. B 737 (2014) 325 [arXiv: 1403.4616] [INSPIRE].

[38] Z. Bern, V. Del Duca and C.R. Schmidt, The infrared behavior of one loop gluon amplitudes at next-to-next-to-leading order, Phys. Lett. B 445 (1998) 168 [hep-ph/9810409] [INSPIRE].

[39] T. Gehrmann and E. Remiddi, Two loop master integrals for $\gamma^{*} \rightarrow 3$ jets: the planar topologies, Nucl. Phys. B 601 (2001) 248 [hep-ph/0008287] [INSPIRE].

[40] T. Gehrmann and E. Remiddi, Two loop master integrals for $\gamma^{*} \rightarrow 3$ jets: the non-planar topologies, Nucl. Phys. B 601 (2001) 287 [hep-ph/0101124] [INSPIRE].

[41] E. Remiddi and J.A.M. Vermaseren, Harmonic polylogarithms, Int. J. Mod. Phys. A 15 (2000) 725 [hep-ph/9905237] [INSPIRE].

[42] T. Gehrmann and E. Remiddi, Differential equations for two loop four point functions, Nucl. Phys. B 580 (2000) 485 [hep-ph/9912329] [InSPIRE].

[43] A.V. Kotikov, Differential equations method: the calculation of vertex type Feynman diagrams, Phys. Lett. B 259 (1991) 314 [INSPIRE].

[44] A.V. Kotikov, Differential equation method: the calculation of $N$ point Feynman diagrams, Phys. Lett. B 267 (1991) 123 [INSPIRE].

[45] J.M. Henn, Multiloop integrals in dimensional regularization made simple, Phys. Rev. Lett. 110 (2013) 251601 [arXiv:1304.1806] [INSPIRE].

[46] S. Moch, P. Uwer and S. Weinzierl, Nested sums, expansion of transcendental functions and multiscale multiloop integrals, J. Math. Phys. 43 (2002) 3363 [hep-ph/0110083] [INSPIRE].

[47] S. Catani, D. de Florian and G. Rodrigo, Space-like (versus time-like) collinear limits in QCD: is factorization violated?, JHEP 07 (2012) 026 [arXiv:1112.4405] [INSPIRE].

[48] A.B. Goncharov, Multiple polylogarithms, cyclotomy and modular complexes, Math. Res. Lett. 5 (1998) 497 [arXiv:1105. 2076] [INSPIRE].

[49] K.T. Chen, Iterated path integrals, Bull. Amer. Math. Soc. 83 (1977) 831 [INSPIRE].

[50] F.C.S. Brown, Multiple zeta values and periods of moduli spaces mathcal $M_{0, n}$, Annales Sci. Ecole Norm. Sup. 42 (2009) 371 [INSPIRE].

[51] A.B. Goncharov, A simple construction of Grassmannian polylogarithms, arXiv:0908.2238 [INSPIRE].

[52] A.B. Goncharov, M. Spradlin, C. Vergu and A. Volovich, Classical polylogarithms for amplitudes and Wilson loops, Phys. Rev. Lett. 105 (2010) 151605 [arXiv:1006.5703] [INSPIRE].

[53] C. Duhr, H. Gangl and J.R. Rhodes, From polygons and symbols to polylogarithmic functions, JHEP 10 (2012) 075 [arXiv:1110.0458] [INSPIRE].

[54] A.B. Goncharov, Galois symmetries of fundamental groupoids and noncommutative geometry, Duke Math. J. 128 (2005) 209 [math/0208144] [INSPIRE].

[55] F. Brown, On the decomposition of motivic multiple zeta values, arXiv:1102.1310 [INSPIRE].

[56] C. Duhr, Hopf algebras, coproducts and symbols: an application to Higgs boson amplitudes, JHEP 08 (2012) 043 [arXiv: 1203.0454] [INSPIRE].

[57] F. Dulat and B. Mistlberger, Real-virtual-virtual contributions to the inclusive Higgs cross section at $N 3 L O$, arXiv:1411.3586 [INSPIRE]. 